Florida International University FIU Digital Commons

3-21-2016

\title{
Co-occurring Health Risks Among Middle Aged Hispanic Men Who Have Sex With Men (HMSM) in South Florida
}

Beatriz Valdes

bvald014@fiu.edu

DOI: 10.25148 /etd.FIDC000243

Follow this and additional works at: https://digitalcommons.fiu.edu/etd

Part of the Family Practice Nursing Commons, Geriatric Nursing Commons, Other Nursing Commons, Psychiatric and Mental Health Nursing Commons, and the Public Health and Community Nursing Commons

\section{Recommended Citation}

Valdes, Beatriz, "Co-occurring Health Risks Among Middle Aged Hispanic Men Who Have Sex With Men (HMSM) in South Florida" (2016). FIU Electronic Theses and Dissertations. 2482.

https://digitalcommons.fiu.edu/etd/2482 


\title{
FLORIDA INTERNATIONAL UNIVERSITY
}

Miami, Florida

CO-OCCURRING HEALTH RISKS AMONG MIDDLE AGED HISPANIC MEN

WHO HAVE SEX WITH MEN (HMSM) IN SOUTH FLORIDA

\author{
A dissertation submitted in partial fulfillment of the \\ requirements for the degree of \\ DOCTOR OF PHILOSOPHY \\ in \\ NURSING \\ by \\ Beatriz Valdes \\ 2016
}


To: Dean Ora Strickland

College of Nursing and Health Sciences

This dissertation, written by Beatriz Valdes, and entitled Co-Occurring Health Risks Among Middle Aged Hispanic Men who have Sex with Men (HMSM) in South Florida, having been approved in respect to style and intellectual content, is referred to you for judgment.

We have read this dissertation and recommend that it be approved.

Eric Fenkl

$\begin{array}{r}\hline \text { Sandra L. Lobar } \\ \hline \text { Michelle Kameka } \\ \hline \text { Soseph De Santis } \\ \hline \text { Sandra Ann Gracia Jones, Major Professor }\end{array}$

Date of Defense: March 21, 2016

The dissertation of Beatriz Valdes is approved.

Dean Ora Strickland
College of Nursing and Health Sciences

College of Nursing and Health Sciences

Andrés G. Gil Vice President for Research and Economic Development and Dean of the University Graduate School

Florida International University, 2016 
(C) Copyright 2016 by Beatriz Valdes

All rights reserved. 


\section{DEDICATION}

This journey has been both challenging and rewarding, with special individuals encouraging and guiding me along the way. I would like to dedicate this dissertation in loving memory of my mother, Luz America Valdes (1925-2013) who was very supportive of my decision to return to school to further my education. Her unconditional love, support and encouragement got me through the first two years of this program. 


\section{ACKNOWLEDGMENT}

Special gratitude goes to my daughter, Chloé Luz Ruiseco, for always understanding why Mami couldn’t go to all the birthday parties or social events. I would not have been successful in completing my dissertation without the tireless support and unconditional love of both my brother, Jorge Valdes and my sister-in-law, Lisa Valdes, whom gracefully assumed the role of Mami when I was in the difficult stages of the doctoral process. Even though we are a small family unit, we show up large when it counts.

I have been truly blessed to have an amazing group of friends as my backbone and I am indebted for all their support. Joseph De Santis helped me stop mourning and get back on track to finish my dissertation. I will be eternally grateful for his support, guidance, and encouragement. Elena Yaber has always been my biggest cheerleader. She has always unconditionally shared her love, support and resources. Sandra Benitez has always been my voice of reason and the formatting queen. Oscar Mickey Arredondo and Guillermo Billy Valdes have always shared their resources, support, and encouragement. Beth Slavin has always provided me with a reality check, which I've needed on many occasions. Lisa Woodson started me on this journey and has always been my esteemed friend, colleague, and travel partner. I am truly indebted to all of these individuals for all of the time, energy, support, encouragement, and love which helped me throughout the dissertation process.

I would also like to extend appreciation to my dissertation committee chair, Dr. Sandra Gracia Jones for her valuable mentorship and encouragement throughout the dissertation process. I would like to thank Dr. Eric Fenkl who willingly shared his 
research and guidance during an immersion course. His 19 transcripts resonated stories of struggles and resilience in older sexual minorities, which resulted in a pivotal change in my dissertation topic. I would also like to thank my dissertation committee members Dr. Eric Fenkl, Dr. Sandra Lobar, Dr. Michelle Kameka, and Dr. Joseph De Santis for their valuable mentorship, encouragement, and continuous guidance. As a recipient of the Sigma Theta Tau International, Beta Tau Chapter PhD Dissertation Research Award, I am grateful for the funding which supported my dissertation research. 
ABSTRACT OF THE DISSERTATION

\title{
CO-OCCURRING HEALTH RISKS AMONG MIDDLE AGED HISPANIC MEN WHO HAVE SEX WITH MEN (HMSM) IN SOUTH FLORIDA
}

by

\author{
Beatriz Valdes
}

Florida International University, 2016

Miami, Florida

\section{Professor Sandra Ann Gracia Jones, Major Professor}

In 2010, men who have sex with men (MSM) represented 4\% of the population in the United States (US) and accounted for 78\% of all new Human Immunodeficiency Virus (HIV) infections among Hispanic men. Hispanic men who have sex with men (HMSM) accounted for the third largest number of new HIV infections (6,700 cases). This dissertation explored the effects of age, loneliness, substance use, depression, and social support on high risk sexual behaviors that predispose middle aged HMSM to sexually transmitted infections (STIs) and HIV infection risk. A sample of 150 urban HMSM aged 40 to 65 were surveyed in this study. Singer's Syndemics Theory (1996) provided this study's theoretical framework. Data was analyzed using a variety of parametric and non-parametric statistics. Loneliness, social support, depressive symptoms, alcohol/drug use, and sexual risk behaviors were found to have an influence on HIV infection status in this study. Partner status, religious affiliation and age did have an influence on alcohol use in this study's participants. Also, participants with increased age had increased depressive symptoms in this study. Lastly, depressive symptoms, substance use, social support, and loneliness did have an influence on sexual risk behaviors in this study's 
participants, specifically alcohol use and illicit drug use. The findings from this study should be used to assess, diagnose, plan, implement and evaluate prevention strategies geared to reduce STI and HIV infection in this population. Future research should build on these findings and develop tailored risk reduction interventions addressing HMSM, with particular attention to the understudied age group of the middle aged HMSM. 


\section{TABLE OF CONTENTS}

CHAPTER

PAGE

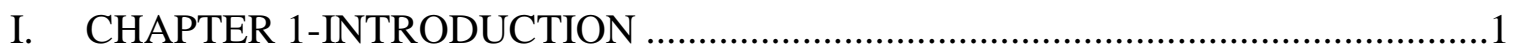

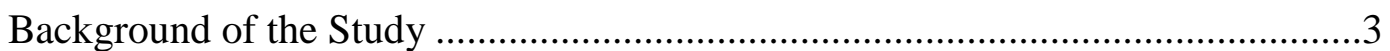

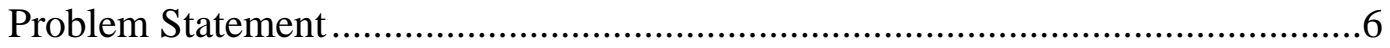

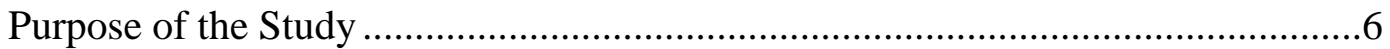

Research Questions ................................................................................................6

Theoretical Framework: Syndemic Theory ……………....................................

Study Assumptions ……………………......................................................10

Definitions of Key Terms ………………………….......................................11

Middle Age ...........................................................................................11

Social Support...................................................................................11

High Risk Sexual Behaviors .....................................................................12

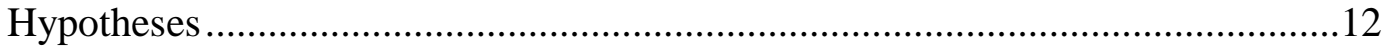

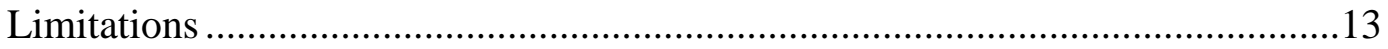

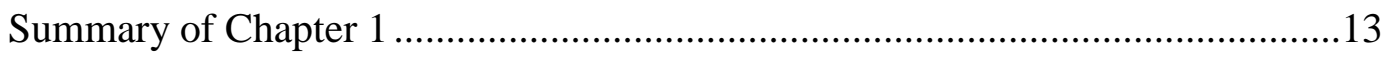

II. CHAPTER 2-REVIEW OF THE LITERATURE _.............................................15

Sources for the Literature Review ..................................................................15

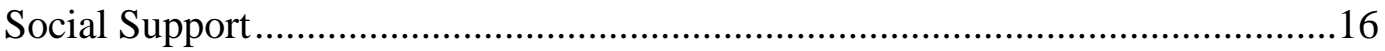

Social Support in Men who have Sex with Men (MSM)........................................16

Social Support in Hispanic Men who have Sex with Men (HMSM) .................... 19

Loneliness ………….............................................................................24

Loneliness in Men who have Sex with Men (MSM) ...........................................24

Loneliness in Hispanic Men who have Sex with Men (HMSM)...........................28

Depressive

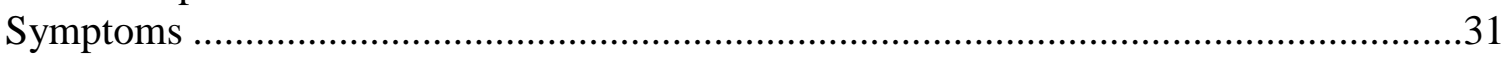

Depressive Symptoms in Men who have Sex with Men (MSM) ............................31

Depressive Symptoms in Hispanic Men who have Sex with Men

Substance

Use/Abuse

Substance Use/Abuse in Men who have Sex with Men (MSM) .............................40

Substance Use/Abuse in Hispanic Men who have Sex with Men (HMSM) ..........42

Sexual Behaviors ...........................................................................................46

Sexual Behaviors in Men who have Sex with Men (MSM) ....................................46

Sexual Behaviors in Hispanic Men who have Sex with Men (HMSM) .................49

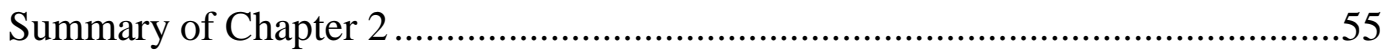




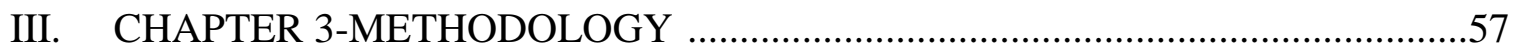

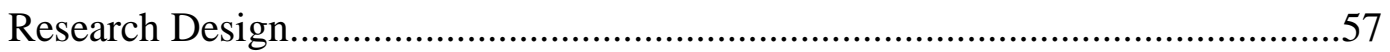

Independent or Predictor Variables .........................................................58

Dependent or Outcome Variables .............................................................58

Attribute Variables ……………………………………......................59

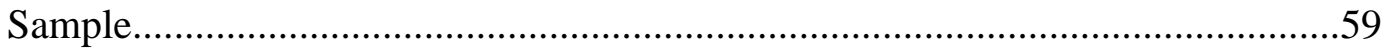

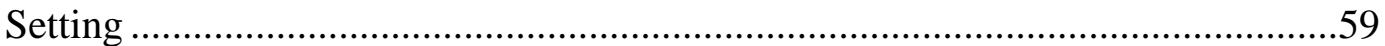

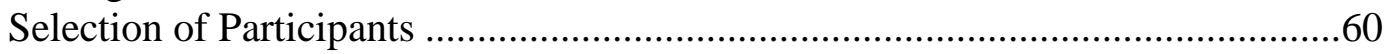

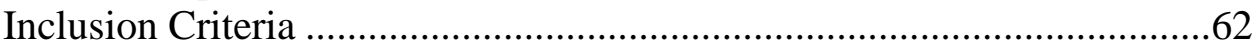

Exclusion Criteria …………………………………………….....62

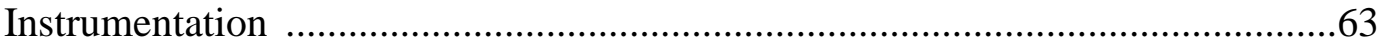

Demographic Questionnaire ..................................................................63

Multidimensional Scale of Perceived Support (MSPSS) Scale..................63

Description of the MSPSS Scale ...................................................64

Norms of the MSPSS Scale ...........................................................64

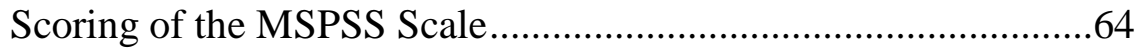

Reliability Data on the MSPSS Scale .........................................65

Validity of the MSPSS Scale .........................................................65

Spanish Version of the MSPSS Scale.............................................66

University of California, Los Angeles (UCLA) Loneliness Scale ............66

Description of the UCLA Loneliness Scale....................................66

Norms of the UCLA Loneliness Scale ………………..................67

Scoring of the UCLA Loneliness Scale .........................................67

Reliability Data on the UCLA Loneliness Scale ...........................68

Validity of the UCLA Loneliness Scale .........................................68

Spanish Version of the UCLA Loneliness Scale ...........................69

Behavioral Risk Assessment Tool (BRAT) ...............................................69

Description of the BRAT ……………........................................

Norms of the BRAT ………………………………..................

Scoring of the BRAT …………………….................................71

Reliability Data on the BRAT......................................................... 71

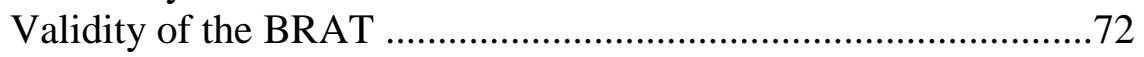

Spanish Version of the BRAT .......................................................72

Center for Epidemiologic Studies Depression (CES-D) Scale ....................72

Description of the CES-D Scale ...................................................72

Norms of the CES-D Scale ..............................................................

Scoring of the CES-D Scale ..........................................................73

Reliability Data on the CES-D Scale .............................................74

Validity of the CES-D Scale .......................................................74

Spanish Version of the CES-D Scale .............................................74

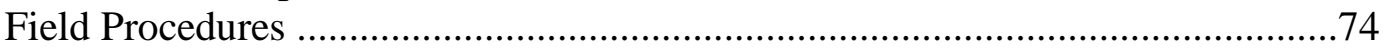

Data Collection \& Recording ............................................................................76

Protection of Human Subjects .....................................................................77

Summary of Chapter 3 .................................................................................79 


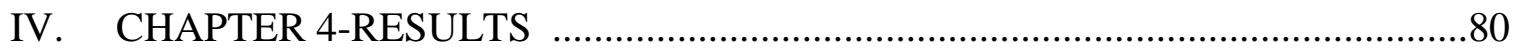

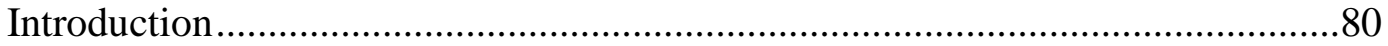

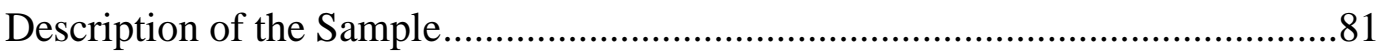

Demographic Characteristics of the Sample.................................................81

Exploratory Data Analyses for Measurement of Variables ...................................84

Measurement Assessments ............................................................................8

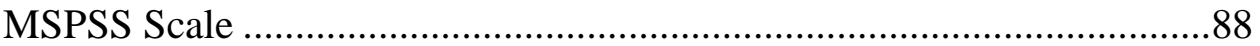

University of California, Los Angeles (UCLA) Loneliness Scale ............88

Behavioral Risk Assessment Tool (BRAT) .................................................89

Center for Epidemiologic Studies Depression (CES-D) Scale ..................91

Descriptive Findings of the Study Variables ........................................................91

Multidimensional Scale of Perceived Support (MSPSS) Scale...................91

University of California, Los Angeles (UCLA) Loneliness Scale ...........92

Behavioral Risk Assessment Tool (BRAT) ...............................................93

Center for Epidemiologic Studies Depression (CES-D) Scale ..................94

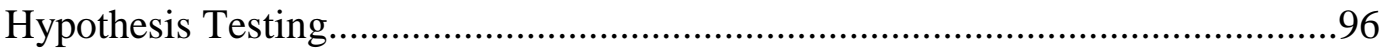

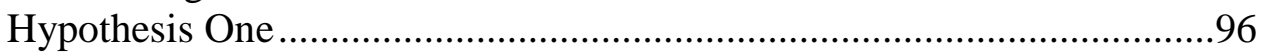

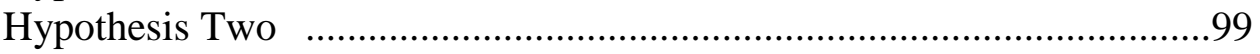

Hypothesis Three ……………………………………….....................102

Summary of Chapter 4 .................................................................................104

V. CHAPTER 5-DISCUSSION AND CONCLUSION ……………........................106

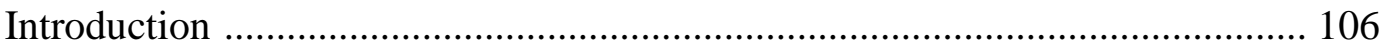

Summary of the Study …………………………….....................................106

Theoretical Framework ……………………………………………....107

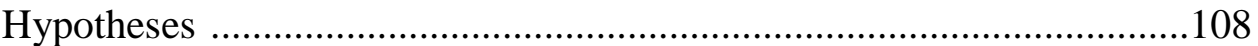

Discussion of the Findings ......................................................................109

Demographic and Background Characteristics............................109

Relationship Between Major Study Variables ..............................111

Limitations of the Study...................................................................................114

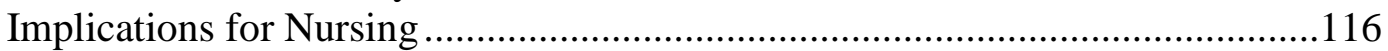

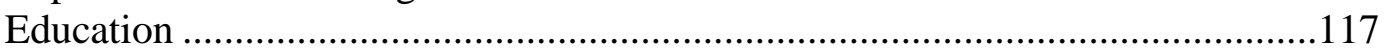

Practice ....................................................................................

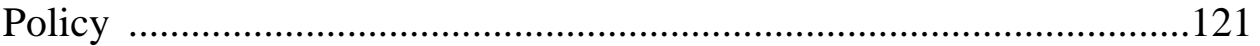

Research ……..........................................................................122

Summary of Chapter 5 .................................................................................. 124

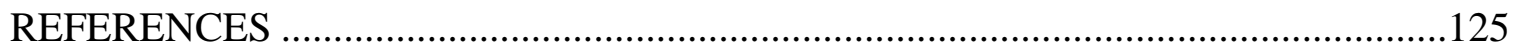

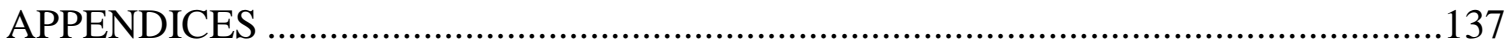

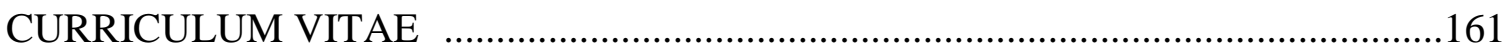




\section{CHAPTER 1-INTRODUCTION}

As demographics change, the United States (US) has become a rapidly growing multicultural population. In 2011, the total population of the US was 301.6 million, which included 38.1 million foreign born persons, representing $12.6 \%$ of the total population; furthermore, nearly half of all foreign-born people in the US are Hispanic (US Census Bureau, 2011). Hispanics account for more than half of the growth in the total population between 2000 and 2010, representing an increase of 56\% and accounting for 70\% of the nation's largest ethnic minority (Center for Disease Control and Prevention [CDC], 2011). The term Hispanic has been defined to be a person of Cuban, Mexican, Puerto Rican, South or Central American, or other Spanish culture or origin, regardless of race (Office of Management and Budget [OMB], 1997).

According to the US Census Bureau (2011), it is estimated that 52 million Hispanics reside in the US, representing approximately $16.7 \%$ of the US total population. Hispanics are projected to more than double in population size between 2000 and 2050, due to fertility and international migration (US Census Bureau, 2011; Ortman \& Guarneri, 2009). Forecasts for 2050 predict that Hispanics are expected to account for 29\% of the population, an estimated 128 million people (Passel \& Cohn, 2008).

Human Immunodeficiency Virus (HIV) is a virus that attacks a key part of the immune system (CD4+ cells or T-cells), leaving the person vulnerable to infections and diseases. When a significant number of CD4+ cells are destroyed, HIV infection has progressed to Acquired Immune Deficiency Syndrome (AIDS). AIDS places a person at risk for opportunistic infections, which may lead to death. There are an estimated 1.2 million people living with HIV infection in the US, and of this group $20 \%$ are unaware of 
their infection status (CDC, 2012a). In 2010, men who have sex with men (MSM) represented $4 \%$ of the population in the US and accounted for $78 \%$ of all new HIV infections among men, of which Hispanic men who have sex with men (HMSM) accounted for the third largest number of new HIV infections (6,700 cases) (CDC, 2013). Hispanics represented $16 \%$ of the population in the US and accounted for $21 \%$ of all new HIV infections (CDC, 2013). In the US, HIV infection was the eighth leading cause of death among Hispanics aged 35-54. Estimates indicate that 1 in 36 Hispanic men will be diagnosed with HIV, and Hispanics are more than 3 times more likely than Caucasians (27.5 vs. 8.7 per 100,000 population) to be diagnosed with HIV infection (CDC, 2013). MSM are also at increased risk for sexually transmitted infections (STIs), including emergence of antimicrobial resistance when compared to their heterosexual counterparts (CDC, 2015). Since the sexual behaviors related with acquiring STIs increase the likelihood of acquiring and transmitting HIV infection, STI incidence among MSM may also be an indicator of higher risk for subsequent HIV infection (Hall et al., 2008). Sexual risk behaviors, such as number of lifetime sex partners, rate of partner exchange, and frequency of unprotected sex, may contribute to rates of STIs and HIV infection among HMSM (CDC, 2015).

Due to continued medical advances, another rapidly growing population is the middle age population. Accounting for 81.5 million (26.4\%) of the people residing in the US, the middle age population (often referred to as the Baby Boom population) who are aged 45 to 64 grew at a rate of $31.5 \%$ (US Census Bureau, 2011). In the US, the average life expectancy in 2011 for men was 76 years, which was shorter than the general 
population life expectancy of 78.7 years (Organization for Economic Cooperation and Development [OECD], 2013).

According to Vance, McGuinness, Musgrove, Orel, and Fazeli (2011), 50\% of HIV infected individuals in the US will be 50 years of age and older by 2017. Incidence rates for HIV infection in individuals 50 years and older is five times higher in Hispanics than in Caucasians (CDC, 2013). Although there are an increasing number of sexually active middle age and older adults, including an increased number living with HIV infection, studies have shown that middle age and older individuals are less likely to be routinely screened for HIV infection (Adekeye, Heiman, Onyeabor, \& Hyacinth, 2012). As a result, middle age and older adults with HIV infection often progress more rapidly when compared to younger individuals (Adekeye et al., 2012).

Estimates for 2011 illustrate that Hispanics continue to experience health disparities in terms of HIV infection. In 2011, Hispanics accounted for 22\% (11,057 cases) of the estimated 50,199 new diagnoses of HIV infection in the US and 84\% (9,256 cases) were in men, of which 79\% (7,266 cases) of the estimated 9,256 cases of HIV infection were attributed to male-to-male sexual contact (CDC, 2013). Unrecognized HIV infection, coupled with lower use of and adherence to HIV medications, places HMSM at increased risk for poor outcomes such as progression to AIDS or death (Hall, Byers, Ling, \& Espinoza, 2007).

\section{Background of the Study}

As a population, HMSM continue to experience health disparities and issues regarding acculturation, substance use and high risk sexual practices that ultimately increase their risk for HIV infection (Gonzalez-Guarda, Ortega, Vazquez, \& De Santis, 
2010). Because Hispanics experience a disproportionate burden of HIV infection and $30.7 \%$ lacked health insurance, HIV infection is a serious threat to the health of the Hispanic community (CDC, 2013). HMSM are disproportionately affected by HIV infection and are at risk for increased mortality and morbidity due to socioeconomic factors, such as lack of access to healthcare, lower educational accomplishments, language barriers, and increased poverty, which limit awareness about HIV infection risks and opportunities for counseling, testing, and treatment (CDC, 2011; CDC 2013).

Great strides have been made in the secondary prevention of HIV infection such as early detection and treatment to delay disease progression to AIDS; however, cultural and socioeconomic factors may place HMSM at increased risk for HIV infection (CDC, 2013). Immigration status, stigma associated with sexual orientation, and/or fear of discrimination are cultural factors that may impede HIV infection reduction efforts (early testing, counseling, or treatment) among HMSM (CDC, 2013; Fernandez, Jacobs, Warren, Sanchez, \& Bowen, 2009). Hispanics are often socially conservative and strongly value familismo, the value of family over the individual resulting in expression of strong loyalty, reciprocity, and solidarity among family members (CDC, 2014). The Hispanic family strongly influences how members perceive themselves (Guarnero, 2013). Sexual behavior issues are considered taboo and gay members within the Hispanic family are often ignored and unsupported, which results in marginalization and risk for depression and suicide (Guarnero, 2007; Guarnero, 2013).

HMSM represent two vulnerable groups (Hispanics and MSM) and account for an $81 \%$ of the estimated $20 \%$ of new HIV infections each year among Hispanics $(9,400$ infections); therefore, there is a critical need to understand this health disparity (CDC, 
2011). Currently, there is no cure for HIV infection, and people with HIV infection in the US place an added burden to healthcare costs in the nation. Despite major advances in diagnosing and treating HIV infection, 35,962 cases of AIDS were diagnosed, and 14,110 deaths among people living with HIV infection were reported in the US in 2007 (CDC, 2011). Furthermore, despite prevention efforts, many HMSM engage in high sexual risk behaviors, predisposing them to HIV infection (Fernandez et al., 2007; Fernandez et al., 2009; Lo, Reisen, Poppen, Bianchi, \& Zea 2011).

From a review of the literature, limited literature was found related to HMSM. Research has found that HMSM engage in high risk sexual behaviors, such as unprotected anal intercourse (UAI) and drug and alcohol use during sexual activity (Fernandez et al., 2007; Fernandez et al., 2009; Lo et al., 2011). Many of the studies revealed co-occurring disorders that precipitate engagement in high risk sexual behaviors, increasing the risk of HIV infection, a health disparity among HMSM (De Santis, Provencio-Vasquez, Weidel, Watson, \& Sanchez, 2009; De Santis, Arcia, Vermeesch, \& Gattamorta, 2011; De Santis, Gonzalez-Guarda, \& Vasquez, 2012; Fernandez et al., 2007; Fernandez et al., 2009). From the existing literature, an apparent knowledge gap was found regarding HMSM, particularly middle aged HMSM, and their associated high risk sexual behaviors. Since there is limited research on HMSM, the proposed study is essential in providing data to direct programs of health education geared to decrease high risk sexual practices among HMSM, which will subsequently reduce morbidity and mortality. Data from this study will be valuable to clinicians, educators and those who make public policy decisions. 


\section{Problem Statement}

Research with HMSM has identified a number of psychosocial issues, such as substance use, depression, loneliness, lack of social connections and support, that are linked to high risk sexual behaviors (De Santis et al., 2009; De Santis et al., 2011; De Santis, Vasquez, Deleon, \& Gonzalez-Guarda, 2012; De Santis, Valdes, ProvencioVasquez, \& Gattamorta, 2015; Fernandez et al., 2007; Fernandez et al., 2009). Despite the available research on high risk sexual behaviors among HMSM, relatively little is known about the sexual risk factors of middle aged HMSM. Therefore, understanding and addressing the risk factors specific to middle aged HMSM (40 to 65 years of age) can guide interventions aimed at reducing HIV infection rates among this vulnerable population.

\section{Purpose of the Study}

The purpose of this quantitative study will be to investigate the effects of age, loneliness, substance use, depression, and social support on high risk sexual behaviors that predispose middle aged HMSM to HIV infection risk.

\section{Research Questions}

The research questions are:

1. Does loneliness, substance use/abuse, depression, decreased social support, and high risk sexual behavior constitute an additive effect for HIV infection susceptibility (syndemic) among middle aged HMSM?

2. What demographic factors among middle aged HMSM (i.e., age, relationship status, religion) are related to loneliness, substance use/abuse, depression, high risk sex, and social support? 
3. How does loneliness, substance use/abuse, depression, and social support influence sexual behaviors of middle aged HMSM?

\section{Theoretical Framework: Syndemic Theory}

First introduced in 1996 by Singer, the concept of syndemics contends that cooccurring health conditions interact synergistically producing adverse health outcomes and inequalities, including susceptibility to disease. The main premise of the Syndemic Theory is based on the assumption that "the interaction of diseases or other health problems (e.g., malnutrition) commonly arises because of adverse social conditions (e.g., poverty, stigmatization, oppressive social relationships) which places socially devalued groups at heightened risk” (Singer et al., 2006, p. 2011). There have been considerable research findings to support the syndemic theoretical approach to understanding HIV infection risk practices among the MSM population (Singer, 2009; Stall et al., 2003; Halkitis et al., 2013; Moeller, Halkitis, \& Surrence, 2011). According to the Syndemic theoretical framework, the presence of two or more psychosocial health problems are additive effects that create a greater increase in HIV vulnerability than one psychosocial problem by itself (Singer, 2009). In 1996, Singer coined the acronym SAVA, which are the interrelations among substance abuse, violence, and AIDS. Furthermore, Singer (1996) proposed that the SAVA syndemic was not only concurrent, but closely linked and interdependent, which when combined with social disparities (e.g. poverty, marginalization) constituted a major syndemic that lead to adverse health outcomes.

The Syndemic Theory has been increasingly used in MSM research focusing on the co-occurring psychosocial health problems and their impact on health outcomes, specifically the risk for HIV infection (Singer, 2009; Stall et al., 2003; Halkitis et al., 
2013; Moeller et al., 2011). In 2003, Stall et al. found "data supporting the view that additive psychosocial health problems, otherwise collectively known as a syndemic, exist among urban MSM and that the interconnection of these problems functions to magnify the effects of the HIV/AIDS epidemic in this population” (p. 941). Stall et al. (2003) telephone interviewed 2,881 men in a cross-sectional household sample of MSM in Chicago, Los Angeles, New York, and San Francisco, who were predominately of European American ethnicity (79\%), but 16\% were older than age 50 years and the majority (84\%) of the sample self-identified as gay. The psychosocial health problems that produced a syndemic effect in Stall et al.'s (2003) study with urban MSM were polydrug use, depression, childhood sexual abuse, and partner violence. In a later study, Stall, Friedman, and Catania (2007) found the possibility of the lifelong effects of experiencing homophobic violence and cultural marginalization at a very early age among gay men as a syndemic process of interacting health problems.

In 2011, findings from Klein’s study with 332 MSM supported the Syndemic Theory and reported that "demographic factors, childhood maltreatment experiences, substance use/abuse, psychological/ psychosocial functioning, and attitudes toward risk taking are all relevant to understanding risk taking, and they are interrelated in a highly complex manner” (p. 475). Moeller et al. (2011) found relationships between mental health, substance use, and sexual behaviors among gay and bisexual men in a sample of 450 club drug-using men from New York City using syndemic theory, whereby, men who had higher risk factors were significantly more likely to engage in sexual risk behaviors. In 2012, De Santis et al. proposed to examine the relationship of eating attitudes and behaviors to alcohol abuse, body image, depression, self-esteem, and sexual 
behaviors, as well as determine the predictors of eating attitudes and behaviors of HMSM using the conceptual framework of Singer's Syndemic Theory. Findings from a study of 100 HMSM with ages ranging from 18 to 51 years suggest that predictors of eating attitudes and behaviors are body image, alcohol abuse, depression, self-esteem, and sexual behaviors, thereby, supporting Singer's Syndemic Theory’s applicability with HMSM (De Santis et al., 2012).

The applicability of the Syndemic Theory was tested with a sample of 199 HIV infected MSM, ages 50 and over, by Halkitis et al. (2013), and found a high level of association between psychosocial burdens (i.e., drug use and mental health) and unprotected sexual behaviors, i.e., UAI. In a study with 504 substance-using MSM (25.4\% Hispanic), Kurtz, Buttram, Surratt, and Stall (2012) found that three syndemic factors (cognitive escape, victimization history, and severe mental distress) predicted HIV infection risk behaviors among HIV negative MSM. Findings from the aforementioned studies suggest the synergistic effects of syndemics, which state that illnesses or risks for illnesses are not mutually exclusive, instead occurring in intersecting clusters.

Singer’s Syndemic Theory (1996) states that addressing one aspect of the syndemic alone is not enough to change or modify health behavior, but intertwined conditions and the social environment in which they occur must be equally considered. Additionally, the theoretical framework of Syndemic Theory captures tightly intertwined circumstances, such as poverty, adequate housing, lack of social support, stigma, substance use, mental health issues, and inequitable healthcare, which when combined lead to health disparities and possible increased health risk behaviors. Even though the Syndemic Theory has not 
been widely used in the MSM population, especially in the aging HMSM population, future HIV research may benefit from taking into account the types of syndemic effects between psychosocial burdens and sexual risk-taking behaviors. The Syndemic Theory provides a better understanding of the additive psychosocial factors and their association with high risk health behaviors.

\section{Study Assumptions}

This study contains both theoretical assumptions and research assumption. The theoretical assumptions were derived from the Syndemic Theory (Singer, 1996). The following is a list of theoretical (1 to 3 ) and research assumptions (4 to 9):

1. Co-occurring health conditions interact synergistically producing adverse health outcomes and inequalities, including susceptibility to disease.

2. Interactions of diseases arise due to adverse social conditions such as stigmatization and place social devalued groups at risk.

3. The presence of two or more psychosocial health problems are additive effects that create a greater increase in STIs and HIV vulnerability than one psychosocial problem alone.

4. The middle age HMSM enrolled in this study will have an appropriate reading and comprehension level.

5. The middle age HMSM in this study will provide honest answers to the research questionnaires.

6. The selected research instruments for measuring the variables actually measure the variables under investigation (DeVellis, 2003). 
7. The chosen methodology is appropriate for answering the research questions (Burns \& Grove, 2009).

8. The data analysis techniques utilized will assist the investigator in analyzing and interpreting the data appropriately (Waltz, Strickland, \& Lenz, 2010).

\section{Definitions of Key Terms}

\section{Middle Age}

Middle age or middle adulthood can be described as the third quarter of the average life span of a person, or the age that is beyond young adulthood, but before the onset of old age. There exist numerous definitions as to the age span for middle age. The US Census Bureau (2011) defines middle age using a chronological age span of 40 to 65 years. For the purposes of the paper, middle age will be defined as the age span between 40 to 65 years of age.

\section{Social Support}

For the purpose of this study, social support will be defined as the strength and number of an individual's social relationships. Additionally, the types of these social relationships will be divided into two constructs, i.e., emotional and/or instrumental. Emotional support refers to the provisions of love and caring, which increases an individual's sense of self worth. Instrumental support refers to the tangible assistance that another provides, such as monetary help and childcare assistance (Zimet, Dahlem, Zimet, \& Farley, 1988).

Social support may be given or received, available or utilized (resource availability), and can be good or bad (quality and quantity). Social support can be a buffer against stressful events and circumstances. Social support networks are not limited 
to family, but include friends and significant others. Social support networks provide the basic need for security, a sense of belonging, companionship and emotional stability (Zimet et al., 1988). Additionally, social support can be informal or formal such as a religious affiliation or social groups. Research with samples of HIV infected older adults and gay men have found social support to be a positive factor in medication adherence, thereby increasing life expectancy for these individuals (Johnson, Heckman, Hansen, Kochman, \& Sikkemma, 2009; Wrubel, Stumbo, \& Johnson, 2008). Research has shown that people who have social support are less vulnerable to ailments and premature death (Cohen, 1988).

\section{High Risk Sexual Behaviors}

An abundance of research demonstrates a consistent association between alcohol and substance use and high risk sexual practices. Research has shown that substance use lowers inhibitions, enhances sexual pleasure, and decreases condom usage (Well, Golub \& Parsons, 2011). According to the CDC (2013), high risk sexual behaviors are behavioral and clinical factors associated with transmission of HIV and other STIs. These behaviors include an individual's history of past STI diagnoses or treatment, substance use (including alcohol, injected and non-injected drugs) and use of non-sterile drug injection equipment, UAI, multiple partners, and sex under the influence of alcohol or drugs.

\section{Hypotheses}

Three hypotheses will be tested in this study with research questions for each as follows: Hypothesis 1: Middle age HMSM (40-65 years of age) with decreased social support and increased loneliness may be at risk for depression and substance use/abuse 
leading to high risk sexual behaviors making them susceptible to HIV infection.

Hypothesis 2: Middle age HMSM with social connections and support are less depressed, lonely, and practice safer sex behaviors.

Hypothesis 3: Middle age HMSM with social connections and support and less psychosocial problems, such as substance use/abuse, depression, and loneliness, report lower levels of high risk sexual behaviors, such as UAI and drug/alcohol use during sexual activity.

\section{Limitations}

For the purposes of this study, the following limitations were applicable:

1. None of the published studies that included HMSM addressed middle age men specifically; therefore, the literature review could not include this specific population.

2. The instruments used in this study have never been used exclusively with the middle age HMSM population.

3. The Behavioral Risk Assessment Tool (BRAT) is an unstandardized instrument with no reported reliability or validity; however, it is a descriptive clinical tool, which provides an idea of individuals' HIV risk behaviors (both sexual and drug/alcohol related behaviors).

\section{Summary of Chapter 1}

High risk sexual behaviors are responsible for the high rates of STIs and HIV infection among HMSM. STIs and HIV infection are infectious diseases that are easily 
preventable provided that individuals do not engage in high risk sexual behaviors, such as UAI and drug/alcohol use during sexual activity (CDC, 2013). Research with HMSM has identified a number of psychosocial factors have been linked to participation in high risk sexual behaviors that could ultimately result in HIV infection (De Santis et al., 2012).

Research with HMSM has shown increases in the number of psychosocial problems, such as substance use, depression, loneliness, lack of social connections and support, are consistent with higher levels of reported high risk sexual behaviors (De Santis et al., 2011; De Santis et al., 2012; Fernandez et al., 2007; Fernandez et al., 2009). The relationship between individual experiences of oppression (e.g., social discrimination and financial hardship) and risk for STIs in the US has been documented for HMSM (Díaz, Ayala, \& Bein, 2004). Additionally, HMSM’s family and social support may be absent related to immigration, or severed due to sexual orientation which may cause loneliness and depression resulting in participation in substance use and high risk sexual behaviors (Meyer \& Champion, 2008; Carballo-Dieguez, et al., 2005). Therefore, understanding and addressing the causes of STIs and HIV infection health disparities among HMSM can guide interventions aimed at reducing these infection rates. This study incorporated the co-occurring relationships between psychosocial burdens and sexual risk taking and addressed co-occurring conditions equally to impact a change in behavior or modify an existing health behavior using the theoretical framework of the Syndemic Theory. 


\section{CHAPTER 2-REVIEW OF THE LITERATURE}

This chapter will present a review and critique of the existing literature of the variables under investigation, i.e., social support, loneliness, substance use/abuse, sexual behaviors, and depressive symptoms of middle age HMSM. This synthesis of the literature will discuss unique characteristics and experiences that affect subsequent high risk sexual behaviors in middle age HMSM, such as engaging in UAI, drug and alcohol use/abuse. Additionally, studies revealed several co-occurring conditions in middle age HMSM that precipitate engagement in high risk sexual behaviors.

\section{Sources for the Literature Review}

Currently, databases with remote access to unlimited number of online journals, books, periodicals, reference materials, and publications are available. For this study, the Louis Calder Memorial Library, which has access to more than 9,000 electronic journals, 400 electronic books, and 150 databases in the biomedical, nursing and allied health sciences was utilized (Louis Calder Memorial Library, 2012). The databases searched were ProQuest, Medline, PubMed, PsycINFO and the Cumulative Index to Nursing and Allied Health Literature (CINAHL) database, which is the comprehensive nursing and allied health research database that provides full text for more than 770 journals (Louis Calder Memorial Library, 2012). Criteria for the literature review included only peerreviewed research based articles in the English language from the year 2010 to present, unless the articles were considered classic manuscripts or pertinent to this study. Research studies, which included other populations, such as women and transgender people, were excluded. 
Initially, the search was conducted without any of the above filters. A Boolean operator “AND” was used to search the literature with the following phrases: men who have sex with men and Hispanic, Hispanic men and high risk behaviors, HMSM and middle age, and Hispanic and MSM and sexual risk. Once filters of year, language and peer review were in place, it provided articles with insight for understanding the current literature on MSM and Hispanics exclusively, as well as determining keyword search terms in the literature. Multiple keyword search combinations for "MSM” were used, such as gay, homosexual, and men who have sex with men. Combinations of keywords used for "middle aged” were older, middle age*, and adulthood; also, keyword "high risk behaviors” was interchanged with “sexual risk.” In addition, other key words searched included: social support, lonely, loneliness, depression, depressive symptoms, substance use/abuse, drug use, alcohol use, and sexual behaviors.

\section{Social Support}

As stated earlier, social support will be defined as the depth and number of an individual's social relationships. Additionally, the types of these social relationships will be divided into two constructs, i.e., emotional and/or instrumental. Emotional support refers to the provisions of love and caring, which increases an individual's sense of selfworth (Zimet et al., 1988). Instrumental support refers to the tangible assistance that another provides, such as monetary help and childcare assistance.

\section{Social Support in Men who have Sex with Men (MSM)}

De Santis and Barroso (2011) used a grounded theory methodology to assess the process of vulnerability in the context of HIV infection with 15 participants of whom $67 \%$ were older than 50 years, $47 \%$ were male, and 53\% were Hispanic. The authors 
defined "vulnerability in the context of HIV infection as the process by which a person with HIV infection is initially confronted with the diagnosis of HIV infection, or diagnosed with an HIV infection related illness in the absence of psychosocial support that is influenced by HIV-related stigma (De Santis \& Barroso, 2011). Confronting mortality and illness, struggling with change, encountering a lack of psychosocial support, and experiencing vulnerability were themes reported from this study. This study highlighted the impact that psychosocial stress has on physical, psychological, and social functioning of people with HIV infection.

Marginalized populations, such as MSM, are disproportionally affected by HIV infection, yet are the least likely to have access to HIV prevention, testing, and treatment services because of widespread stigma and discrimination. The loss of psychosocial support combined with the physical, psychological, social, and spiritual changes that occur with HIV infection may result in physical weakness, psychological helplessness, social isolation, and spiritual distress, and place HIV infected individuals at risk for increased physical and psychological morbidity (De Santis \& Barroso, 2011). Since HIV infection remains a highly stigmatized condition and continues to be blamed on irresponsible personal behavior, HIV infected individuals may have feelings of stigma and marginalization. A concern with this study is the small sample size and the large number of Hispanic participants; hence, limiting the ability for generalization to the MSM population.

In a qualitative study to describe the practical support for antiretroviral therapy (ART) adherence offered by partners of MSM with HIV infection by Wrubel et al. (2008) 20 couples were interviewed. Ten couples were seroconcordant and 10 were 
serodiscordant. The 30 participants with HIV infection were taking ART for an average of 7 years. Mean age for participants was 48.7 years $(S D=9)$. The ethnic characteristics for the sample included 70\% $(n=28)$ Caucasian, 15\% $(n=6)$ African American, 10\% Hispanic ( $n=4)$, and 5\% $(n=2)$ other. The qualitative interviews focused on the narratives concerning what practical support for adherence the participants offered their partners with HIV infection and the narratives recounting what support for adherence the participants with HIV infection perceived receiving (Wrubel et al., 2008). The study findings demonstrated a range of social support practices for ART adherence. Partners reminded, monitored medications, organized medications, ordered medications and coached their significant HIV infected other by reinforcing the gains of medication adherence. A concern with this study is that it surveyed a small number of MSM participants (40) and the participants were primarily Caucasian, which is not representative of the general population of MSM.

In a study by Johnson et al. (2009), two ART adherence conceptual frameworks were examined with $244 \mathrm{HIV}$ infected older participants as part of a randomized clinical trial (RCT) for a coping improvement intervention. The sample characteristics included 49.2\% ( $n=120)$ African Americans, 30.3\% ( $n=74)$ Caucasians, $14.4 \%(n=35)$ Hispanics, and 6.2\% $(n=15)$ other, of which $70.9 \%$ were male and 57\% $(n=139)$ were gay/lesbian/bisexual. Participants had a mean age of $55.5(S D=4.8)$, with ranges from 50 to 73 years. Social support was measured using the Functional Assessment of Chronic Illness Therapy (FACIT) and the Provision of Social Relations Scale (PSR). Using SEM results supported the notion that affect mediates the relationships among social support, maladaptive coping, and adherence $($ FIML $\chi 2(56, N=244)=94.81, p<0.001$, RMSEA $=$ 
$0.05(\mathrm{CI} 90 \%=0.03,0.07)$, and all of the structural paths were statistically significant $(p$ $<0.001$ ). This model accounted for $70 \%$ of the variance in negative affect, $16 \%$ in adherence, and 21\% in HIV viral load (Johnson et al., 2009). Additionally, social support $(\gamma=-0.47, p<0.001)$ and maladaptive coping $(\gamma=0.46, p<0.001)$ was found to predict negative affect, which predicts adherence $(\beta=-0.34, p<0.001)$ (Johnson et al., 2009). Therefore, findings suggest increased adherence to ART in HIV infected older adults increase positive affect, and enhanced adaptive coping and social support. This study supports previous findings, which suggest social support has a positive effect on physical and emotional health, making it an important health determinant. Since this study is not exclusively MSM, it is limited for generalization to that population.

\section{Social Support in Hispanic Men who have Sex with Men (HMSM)}

Hispanics are often socially conservative and strongly value familismo, the value of family over the individual resulting in expression of strong loyalty, reciprocity, and solidarity among family members (CDC, 2014). Sexual behavior issues are considered taboo and gay members within the Hispanic family are often ignored and unsupported, which results in marginalization, decreased social support, and risk for depression and suicide (Guarnero, 2007; Guarnero, 2013). In an editorial addressing HMSM interventions, Ramirez-Valles (2007) discussed the importance of bonds with family, friends, peers, and the Hispanic community, which fosters innate trust, obligation, solidarity, and compañerismo (collegiality).

Substance abuse was found as a means to buffer negative events or emotions, such as loneliness, separation from family (due to migration or marginalization), and loss of social support for HMSM (De Santis, Gonzalez-Guarda, Provencio-Vasquez, \& Deleon, 
2014). HMSM reported internal (within the Hispanic family and community) and external discrimination for both ethnic identification and sexual orientation (De Santis et al., 2014). Since HIV infection and homosexuality is highly stigmatized and marginalized within the Hispanic family, HMSM attempt to conceal sexual orientation and HIV infection status (De Santis et al., 2014; Guarnero, 2007). Findings from De Santis et al. (2014) reported the lack of psychosocial support for HMSM resulted in high risk sex, substance abuse, and violence, consequentially increasing risk for HIV infection. Therefore, screening for psychosocial stressors, such as discrimination, stigma, and marginalization, in HMSM can decrease HIV infection rates.

Fernandez et al. (2007) hypothesized that crystal methamphetamine users would report more HIV infection related sexual risk behaviors, were likely to have attempted suicide, had less social connections, more psychological distress, higher orientation to American culture, lower orientation to Hispanic culture, and reported higher gay community attachment than men who did not use the drug. The study sample included 566 HMSM with a mean age of 31years who were separated into three categories: non drug user $(n=325)$, non-crystal methamphetamine user but may use other recreational drugs ( $n=184)$, and crystal methamphetamine users $(n=57)$ (Fernandez et al., 2007).

Participants completed a computer assisted self-interview lasting 60- to 90minutes, which found high rates of crystal methamphetamine use associated with acculturated HMSM (Fernandez et al., 2007). Crystal methamphetamine users were more likely than non-drug users to have lower orientation to Hispanic culture (Hispanicism), higher attachment to the gay community, higher rates of UAI, and a history of suicide attempts. Szapocznik, Kurtines, and Fernandez (1980) developed the 
Behavioral Acculturation Scale and identified the terms Hispanicism and Americanism as concepts that were measured as subscales related to bicultural identification, whereby Americanism signifies the loss of Hispanic culture and greater acculturation. The strength of this study was the multidimensional relationship between crystal methamphetamine use and high risk sexual behaviors among HMSM.

As a follow up to their first study in 2007, Fernandez et al. (2009) researched psychological factors associated with drug use and illustrated how risk and protective factors associated with drug use and high risk sexual behaviors guided development of Proyecto SOL, a theory-based, culturally tailored HIV risk reduction intervention for HMSM. The study was a mixed method design with both quantitative and qualitative data. For the qualitative portion, the authors interviewed 20 participants, who were $80 \%$ ( $n=16)$ HIV negative or of unknown serostatus and 75\% $(n=15)$ of them used drugs and had high risk sexual behaviors (Fernandez et al., 2009). The quantitative portion collected data on sociocultural and psychological factors associated with drug use and high risk sexual behavior among 566 HMSM (Fernandez et al., 2009).

For the quantitative analyses, participants were classified as drug users (43\%) or non-drug users (57\%) based upon their self-reported 6-month history of using the following drugs: cocaine, crystal methamphetamine, other amphetamines, poppers, ecstasy, GHB, Special K, and Viagra in combination with other drugs. Drug users were more likely than non-drug users to be US born, interviewed in English, have higher monthly incomes, been previously tested for HIV infection, and test seropositive for HIV infection (Fernandez et al., 2009). Drug users were more oriented to American culture (Americanism) and less Hispanicism than non-drug users (Fernandez et al., 2009). Drug 
users reported higher levels of loneliness, psychological distress, experiences of discrimination, more sex partners, and higher rates of UAI, but less social connections (Fernandez et al., 2009).

In the qualitative phase, the study included the following research questions: (a) relationship and role of drugs and sex; (b) context and motivation of drug use and sexual behaviors; (c) role of Hispanic and gay culture in drug use and sexual behaviors; (d) role of social support in risk and protection; (e) issues related to HIV intervention development (Fernandez et al., 2009). Fernandez et al. (2009) found themes of a need for acceptance and desire to please partners emerged as core drivers of HIV infection risk for the HMSM. The following four main themes emerged from Fernandez et al.’s (2009) study: quest for acceptance from partners and family often led to risky sex and/or drug use; strong association between drug use and risky sex even among men who were casual or infrequent users; cultural influences and the often conflicting messages men receive from the Hispanic, the mainstream American, and the gay cultures; and loneliness and the need for social connections. Interestingly, loneliness was a theme identified even though the study took place in South Florida, which has a large gay community with many social networks (Fernandez et al., 2009).

In Lauby et al.'s (2012) study, the authors hypothesized that Black and Latino MSM who have supportive social relationships with other people are less likely to have unrecognized HIV infection compared with MSM who report lower levels of social support. Data for this analysis were collected as part of the Brothers y Hermanos (ByH) study of Black and Latino MSM, sponsored by the CDC. The study did not identify any specific theoretical or conceptual framework. A total of 2,235 Black and Latino MSM 
completed the interview at the four sites. HMSM participants were recruited in New York City $(n=516)$ and Los Angeles County $(n=565)$. Participants completed a computer administered questionnaire and were tested for HIV infection. Unrecognized HIV infection was found in 118 men (9.2\%). MSM who scored higher on the supportive relationship index had significantly lower odds of testing HIV antibody positive in the study, $A O R=0.68$ (Lauby et al., 2012). The mediation analysis identified two possible behavioral pathways that may partially explain this association after controlling for race/ethnicity, age, annual income, sexual identity and network size, i.e., men who had strong supportive relationships were more likely to have had a test for HIV infection in the past 2 years, and less likely to have recently engaged in high risk sexual behavior (Lauby et al., 2012). Limitations of the study include using a cross-sectional survey, which does not test causal paths between supportive relationships and unrecognized HIV infection. Also, measures of recent sexual risk behavior (past 3 months) may not have covered the time period in which HIV infection occurred. This study supports the link between supportive relationships and HIV infection determinants.

\section{Summary of Social Support in the Literature}

From a synthesis of the literature, only four research studies were found relating to HMSM and the effects of social support, but none were exclusively middle aged HMSM (De Santis et al., 2014; Fernandez et al., 2007; Fernandez et al., 2009; Lauby et al., 2012). Additionally, all of the findings referenced marginalization, substance use/abuse, high risk sexual behaviors and risk for HIV infection for HMSM who lacked social support. Research studies found with the general population of MSM all referenced HIV infected individuals and the positive role of social support in medication 
adherence, thereby increasing life expectancy for these individuals (Johnson et al., 2009; Wrubel et al., 2008). Social support can be a buffer against stressful events and circumstances. Research has shown that people who have social support are less vulnerable to ailments and premature death; therefore, this apparent gap in the literature with middle age HMSM should be addressed (Cohen, 1988).

\section{Loneliness}

Loneliness is an emotional state of mind in which an individual has feelings of emptiness, undesirability, and isolation. Individuals who are lonely often feel cut-off, disconnected, and alienated from those around them. Individuals may experience loneliness as a response to different life events, i.e., immigration, loss of important relationships (death, divorce or separation), and perceptions of internalized homophobia or stigma (Russell, Peplau, and Ferguson, 1978).

\section{Loneliness in Men who have Sex with Men (MSM)}

According to Torres and Gore-Felton (2007), identity development for MSM (attraction to same-sex individuals) during the developmental milestone is strongly influenced by feelings of being different, if homosexuality is negatively perceived. This often exacerbates isolation and feelings of loneliness. Therefore, MSM may develop feelings of internalized homophobia and stigma, coupled with a loss of social support, which results from being part of a marginalized group. Additionally, research has shown that large portions of MSM do not have family support (Carballo-Dieguez et al., 2005; Torres \& Gore-Felton, 2007). Hence, many MSM are faced with choosing between maintaining family support by living a "double life" (hiding their sexual orientation) and 
living an openly gay life without the support of their families (Carballo-Dieguez, 2005; Torres \& Gore-Felton, 2007). This may increase MSM’s vulnerability to loneliness.

The Loneliness and Sexual Risk Model (LSRM) is based on a theoretical framework developed for MSM, which hypothesizes that loneliness is associated with sexual risk behavior, mediated by the influence of substance use and compulsive behavior (Torres \& Gore-Felton, 2007). Research with the LSRM model was conducted with a convenience sample of 49 adolescents, with ages ranging from 16 to 19 years old $(M=17.84, S D=1.01)$, seeking services at a Lesbian, Gay, Bisexual, and Transgender (LGBT) Wisconsin community center (DeLonga et al., 2011). The sample was comprised of 94\% self-identified MSM who were predominately African American (69\%), Caucasian (10\%), Hispanic (2\%), and other (18\%). Participants reported up to 40 sexual partners in the past year $(M=4.5, S D=6.9)$ and loneliness was found to be positively and significantly associated with compulsive Internet use $(r=.46, p<.001)$. MSM who experienced greater feelings of loneliness and more internalized homophobia reported greater numbers of sexual partners during the past year (DeLonga et al., 2011). Therefore, loneliness was found to influence MSM's decisions regarding sexual behavior with partners of unknown HIV infection status. A concern with this study is that it surveyed a small number of African American participants and used a convenience sample, which is not representative of the general population of MSM.

Using the Self-Justification Questionnaire (SJQ), Skinta, Murphy, Paul, Schwarcz, and Dilley (2012) studied factors that identified rationales (thoughts, attitudes, and beliefs) for continued participation in UAI with MSM partners of unknown or discordant serostatus (one partner has a known HIV infection and the other does not). 
Study participants included 182 HIV infected MSM from multiple sites across San Francisco. The sample was comprised of 41.8\% Caucasian, 26.4\% African American, 18.1\% Hispanic, and $13.7 \%$ others. The participants’ age was reported in 10-year categories. Predominately, the sample was comprised of participants who were 31 years of age and above (96.7\%), of which ages 41 to 50 years (57.1\%) accounted for the majority. Participants reported a mean of 13.8 anal sex partners in the previous 12 months (SD not reported). Results from this descriptive study with HIV infected MSM, endorsed items relating to the importance of intimacy or loneliness for UAI decisionmaking. Participants endorsed the item related to intimacy and emotional needs (52.7\%), i.e., "I've been so lonely-being with this guy gives me that feeling of connecting with someone that I need so much,” as the reason for UAI (Skinta et al., 2012). Therefore, in an effort to gain temporary relief, individuals who are intimately and socially isolated may be less likely to negotiate condom use during sexual activities (Skinta et al., 2012). This study is not representative of the population of MSM since it had a small sample size and the majority of participants were 41 to 50 years old.

Hubach et al. (2015) studied factors of loneliness, HIV infection related stigma, and condom use in a sample of $100 \mathrm{HIV}$ infected MSM residing in rural Indiana. The samples' mean age was $42.60(S D=11.82)$. The composition of the sample was $86 \%$ Caucasian, 6\% Hispanic, 4\% African American, and 3\% other. Sexual behaviors were reported as follows: $30.2 \%$ of participants $(n=13)$ utilized condoms with their most recent HIV infected partner, 53.57\% $(n=15)$ utilized condoms with most recent non-HIV infected partner, and 23.5\% $(n=4)$ utilized condoms with most recent partner of unknown HIV infection serostatus. The revised $3^{\text {rd }}$ version of the UCLA Loneliness 
Scale was used to explore the intercorrelations between loneliness, total HIV stigma, and safer sex attitudes of the participants. Findings indicate that HIV infection stigma was highly correlated with loneliness $(r=0.619, p<0.01)$ and loneliness negatively predicted condom use with the most recent partner of unknown HIV infection status. Furthermore, it was reported that for every unit increase in the loneliness scale score, a 10.3\% increase in UAI will occur with partners of unknown HIV infection serostatus, exponentiated coefficient $(\exp B)=0.897,95 \%$ CI [0.807, 0.998], $p<0.05)$ (Hubach et al., 2015). Therefore, experiences of social isolation and feelings of loneliness can exacerbate high risk sexual behaviors. A limitation of this study is the location where it took place (rural Indiana) and the sample was comprised of mostly Caucasians, which is not representative of the population of MSM.

In an exploratory study identifying social service needs for older self-identified gay men, lesbians and bisexuals, Jacobs, Rasmussen and Hohman (2008) recruited 71 participants. Participants' age ranged from 50 to 80 years $(M=60.8, S D=8.31)$. The sample comprised of mostly male participants $(76.1 \%, n=54)$ and approximately oneforth female (23.9\%, $n=17)$. Among the 71 participants, 32 (45.7\%) were retired, 33 (47.1\%) were single and not in a relationship, 26 (37.1\%) were in a committed relationship, and 7 (9.8\%) were married with same sex partner, divorced or widowed. Housing and living arrangements were reported as follows: 36 (50\%) lived alone, 23 (32.9\%) lived with a partner or lover, and 12 (17.2\%) lived with roommates or family. The sample was predominately Caucasian ( $n=61,85.9 \%)$, but included three (4\%) Hispanics, three (4\%) Native Americans, three (4\%) other, and one (2.1\%) Asian. After exploring problems faced by older gay men and lesbians, participants ranked loneliness 
$(16.9 \%, n=12)$ as the second to the largest problem (income $23.9 \%, n=17)$. Twentytwo percent $(n=16)$ of participants reported age discrimination from society in general and remarkably 34.3\% $(n=24)$ reported age discrimination within the lesbian and gay community.

\section{Loneliness in Hispanic Men who have Sex with Men (HMSM)}

Even when voluntary, immigration to another country is often difficult. Separation from familiar societal norms, family, and cultural practices make it difficult for foreign-born HMSM to adapt and may result in loneliness. Additionally, cultural prohibitions against same-sex partnerships in HMSM often result in feelings of internalized homophobia (negative beliefs about same-sex attraction) and stigma (Carballo-Dieguez et al., 2005). Social connections with family, friends and community may be disrupted due to this internalized homophobia among HMSM, resulting in loneliness.

Rhodes et al. (2011) designed a community based participatory research (CBPR) partnership in North Carolina to establish prevalence estimates of HIV infection risk behaviors among MSM. Using a grounded theory approach, nine focus groups were conducted totaling 88 MSM participants. Participants mean age was 27 years (ranged from 18 to 60 years). The sample was comprised of 31\% African American $(n=28)$, 38\% Hispanic ( $n=33), 24 \%$ White $(n=21)$, and 7\% other $(n=6)$. One of the main 12 themes related to HIV infection risk that emerged from the sessions for the HMSM group was the reported high levels of loneliness and social isolation. Similar to previous research with HMSM, fear of discovery and cultural prohibitions for engaging in samesex behavior isolated HMSM from each other and their communities (Carballo-Dieguez 
et al., 2005; De Santis et al., 2014; Guarnero, 2007). Additionally, themes of language and cultural barriers, distance and lack of physical spaces to congregate emerged (Rhodes et al., 2011). This study would've been more informative if it surveyed each ethnic group separately.

As part of a larger study, De Santis et al. (2014) reported findings from a qualitative grounded theory approach study in South Florida, which included HMSM ( $n=$ 20). The sample participants were foreign-born, predominately from Cuba $(n=9,45 \%)$ and had a mean age of $43.60(S D=8.61)$. Four focus groups conducted in Spanish gathered perceptions and concerns regarding high risk sexual behaviors, substance use/abuse, and violence in the HMSM community. HMSM reported internal (within the Hispanic family and community) and external discrimination for both ethnic identification and sexual orientation. Themes regarding negation (denial) of sexual risk, substance abuse as a buffer, and the burden of violence saturated the authors' findings. Participants also reported a lack of psychosocial support. Because homosexuality is highly stigmatized and marginalized within the Hispanic family, HMSM who openly disclose their sexual orientation may be disowned and banished resulting in feelings of loneliness and alienation (De Santis et al., 2014; Guarnero, 2007). Additionally, substance abuse was found as a means to buffer negative events or emotions, such as loneliness, separation from family (due to migration or marginalization), and loss of social support for HMSM (De Santis et al., 2014). Findings from De Santis et al. (2014) reported the lack of psychosocial support for HMSM resulted in high risk sex, substance abuse, and violence, consequentially increasing risk for HIV infection. Since it was implied that marginalization from the Hispanic family may result in feelings of 
loneliness, themes of loneliness were not exclusively found, this presented as a limitation of the study. Additionally, the sample was predominately Cuban HMSM, which may not truly be representative of the population of HMSM. The major contribution of this study is the findings, which include screening for psychosocial stressors, such as loneliness, discrimination, stigma, and marginalization, in HMSM can decrease HIV infection rates in this group.

\section{Summary of Loneliness in the Literature}

From this review of the literature, no studies were found on middle age HMSM and loneliness. Based on the research studies that were included in this literature review, two studies were found regarding HMSM and loneliness (Rhodes et al., 2011; De Santis et al., 2014). From these studies it can be concluded that middle age HMSM may be at risk for loneliness, but the results of these studies could be questionable due to such small sample sizes (20 to 33 participants). Small sample sizes increase the chance of sampling errors, thereby not accurately representing the population from which the sample is drawn (Polit \& Beck, 2012).

Since very few studies have examined the effects of loneliness on HMSM's sexual behaviors, loneliness with the general population of MSM was explored. This resulted in three studies where loneliness was the theme or variable of measurement. However, the results of these studies can also be questionable due to several issues. These studies had small sample sizes (49 to 182 participants), thereby increasing the chances of sampling error. The ethnic composition of the participants was not indicative of the general population of MSM, and some of the locations of the studies were remote. Additionally, most of the research found with MSM and HMSM and loneliness, grouped 
loneliness into negative mood states (e.g., anxiety, depression), instead of actually measuring loneliness with a scale. However, the studies did examine the effect of loneliness on high risk sexual behaviors. This is an important finding because it is hypothesized that lonely middle age HMSM who are seeking to reduce their feelings of loneliness are more likely to use/abuse alcohol and drugs, and engage in high risk sexual behaviors, placing them at risk for HIV infection.

\section{Depressive Symptoms}

Major depressive disorder (MDD) is a disabling condition characterized by a pervasive feeling of sadness and loss of interest in normally enjoyable activities, which negatively impacts the quality of life and adversely affects an individual's behavior, sleep and eating patterns, and general health (Jarvis, 2012). Depressed individuals have shorter life expectancies due to illnesses and suicide. MDD often co-occurs with increased rates of alcohol and drug abuse and particularly dependence (Arseniou, Arkaterini, \& Samakouri, 2013; De Santis, 2013).

\section{Depressive Symptoms in Men who have Sex with Men (MSM)}

In a longitudinal multi-site study, O’Cleirigh et al. (2013) explored 746 HIV infected MSM. Participants mean age was $41.9(S D=8.5)$. The sample characteristics included 63.3\% Caucasian, 23.4\% African American, 9.9\% Hispanic, and 3.4\% other. Different instruments were used to measure depression in the six study sites. The CES-D was used at three locations including an abbreviated version at one of the three locations. The other three locations used a different tool at each one, i.e., the subscale from Profile of Mood States (POM), Patient Health Questionnaire (PHQ) and the Beck Depression Inventory (BDI). Findings reported that older age was associated with a significant 
reduction in the odds of sexual risk over time $(O R=0.99 ; 95 \% C I$ : $0.996-0.999, d f=$ $741, p=0.004)$, and decrease per month in the odds of both sexual risk for those with moderate depression $(O R=0.95: 95 \% C I: 0.93-0.98, d f=740, p<0.001)$. Sexual risk was associated with lower depression $(O R=0.97: 95 \% C I$ : 0.94-0.99, $d f=740, p<$ 0.05). Additionally, a decrease in the odds of sexual risk associated with greater depression was also significant $(O R=0.97: 95 \% C I$ : 0.96-0.98, $d f=740, \mathrm{p}<0.05)$. Therefore, 12 months of follow findings indicate moderate levels of depression are associated with less improvement over time in the odds of engaging in sexual risk in HIV-infected MSM, as opposed to those who have low and high levels of depression. This study adds to the scientific knowledge regarding the association between depression and sexual risk in MSM, if HIV antibody positive. A limitation of the study is that the researchers used various instruments to measure the same concept of depression and this could result in erroneous findings. Another limitation of this study is the actual cause of depression for MSM, which could be related to HIV infection status or not.

In an Internet study by Blashill and Vander Wal (2010) 162 gay men were recruited to explore the impact of gender role conflict (GRC) as a mediator for the relationship between social sensitivity and depression. The sample participants were 76\% $(n=123)$ Caucasian, 9\% $(n=15)$ multiethnic, 6\% $(n=10)$ Hispanic, 6\% $(n=10)$ Asian, 2\% $(n=3)$ African American, and 1\% $(n=1)$ Persian. Participants mean age was reported as $32.26(S D=13.01)$, ranging from 18 to 75 years. The study's theoretical framework was defined by GRC, which is based on the premise that men develop intrapersonal conflict when exposed to contrasting norms concerning appropriate societal gender roles and behaviors. The GRC scale measured reactions to the gender role 
expectations men face in society with four subscales: Success/Power/Competition; Restrictive Emotionality; Restrictive Affectionate Behavior between Men; and Conflict between Work and Family Relations (Blashill \& Vander Wal, 2010). The Brief Fear of Negative Evaluation scale (BFNE) was used to measure social sensitivity (concerns about receiving disapproval from others). The CES-D was used to measure depression. The authors employed the use of non-parametric bootstrapping procedures to determine the influence of GRC on the relationship between social sensitivity and depression. After controlling for age, results indicated that the total indirect effect mediated the relationship between social sensitivity and depression (Blashill \& Vander Wal, 2010). Furthermore, the indirect effects of Restrictive Emotionality, Restrictive Affectionate Behavior between Men, and Conflict between Work and Family Relations were stronger than that of Success/Power/Competition. Therefore, the combined effects of the components of GRC mediated the relationship between social sensitivity and depression. A predominately Caucasian sample is this study's limitation. This study provides insight as to how gay men may expend excessive amounts of energy in attempts to appear heterosexual in certain social situations and by doing so may result with depression.

In a four-year study with MSM who engage in bareback sex (intentional condomless sexual activity with risk for HIV infection transmission), Houston, Sandfort, Dolezal, and Carballo-Dieguez (2012) recruited 120 participants to explore the effect of depressive symptoms on rates of barebacking sexual activities. The participants mean age was 34 years $(S D=9.6$ years) and reported years of education was 14.8 years $(S D=$ 2.9). Among the 120 MSM, 26\% ( $n=31$ ) were HIV infected, 29.2\% were Caucasian, 
25.8\% were Hispanic, 23.3\% were African-American, and 14.2\% were Asian/Pacific Islander.

Ninety-six percent of participants $(n=115)$ reported UAI within the last two months, with a mean of 6 occasions and interquartile range of 11 occasions. Depressive symptoms were assessed using the Beck Depressive Inventory scale (BDI-II), which is a 21-item instrument with two subscales (cognitive-affective and somatic) that yields a score, and higher scores indicate greater depressive symptoms (Beck, Steer, \& Brown, 1996). Total BDI-II scores were significantly correlated with the number of UAI occasions with a potentially serodiscordant partner for the non-HIV infected men in the study (Spearman's rho $=.23, p<.05$ ). Specifically, the cognitive-affective subscale of depressive symptoms was significantly correlated with UAI occasions with serodiscordant partners (Spearman's rho $=.24, p<.05$ ), but the somatic subscale was not significant. Significant differences in all the men in the sample were found regarding depressive symptoms (using the cognitive-affective subscale only) and men who engaged in serodiscordant UAI versus those who $\operatorname{did} \operatorname{not}(\mathrm{Z}=-2.309 ; p=.029)$. Using the total BDI-II score, the non-HIV infected individuals who engaged in discordant UAI reported greater levels of depressive symptoms than the non-HIV infected participants who did not engage in discordant UAI (Ms $=13.5$ vs. $7.9, \mathrm{Z}=-2.183 ; p=.027)$. After controlling for demographic factors (i.e., race/ethnicity, income, and education), Houston et al. (2012) found all participants who engaged in serodiscordant UAI were significantly more likely to report greater depressive symptoms (based on the cognitive-affective subscale), Wald $(1, n=120)=4.85, p=.028$. 
Additionally, after controlling for demographic factors, non-HIV infected men who engaged in serodiscordant UAI were more likely to report greater depressive symptoms, Wald $(1, n=83)=4.49, \mathrm{p}=.034$. Even though limitations did exist in the study, such as measuring depressive symptoms only within the past two weeks as opposed to measuring sexual behaviors within the past two months and the small sample size, Houston et al. (2012) provided a link between sexual risk taking and depressive symptoms among MSM. Findings suggest that there is a direct relationship between depressive symptoms and sexual risk taking for non-HIV infected MSM, with greater depressive symptoms corresponding to an increased frequency of bareback sex.

\section{Depressive Symptoms in Hispanic Men who have Sex with Men (HMSM)}

In a descriptive design study of 152 participants ranging in age from 21 to 58 years ( $M=36.7, S D$ not reported), De Santis et al. (2009) explored the relationship of depressive symptoms, self-esteem, and sexual behaviors in HMSM. The sample characteristics included HMSM who were foreign-born $(87.5 \%, n=133)$ and those who were US-Born $(12.5 \%, n=19)$. Instruments used to collect data were the following: the CES-D was used to collect measures on depression, the Rosenberg Self-Esteem Scale (RSES) collected measures on self-esteem, and the Safer Sex Behaviors Questionnaire (SSBQ) collected data on sexual behaviors. The authors designated the total score for the CES-D of 16 or above to indicate higher levels of depression. Total CES-D scores ranged from 0 to $54(M=15.1, S D=10.7)$ for the entire sample, $55(35.5 \%)$ of the participants had CES-D scores above 16. The authors used a total score of 26 and above to indicate higher levels of self-esteem. The total RSES scores ranged from 16 to 40 ( $M$ $=26.2, S D=3.4)$, and $83.9 \%(n=130)$ of the participants had RSES scores less than 26, 
indicating lower levels of self-esteem. The authors used scores of 78 or less to indicate lower levels of safer sex behavior and scores greater than 78 indicated higher levels of safer sex behavior. The total SSBQ scores ranged from 46 to $104(M=79.3, S D=12.6)$, 71 (45.8\%) had SSBQ scores less than 78, indicating lower levels of safer sex behaviors. Chi-square analyses reported differences between foreign-born and US-born on the three variables. Foreign-born HMSM had less depressive symptoms when compared to USborn HMSM, $\left.\chi^{2}(37, n=155)=59.5, p=.011\right)$. Additionally, sexual behaviors of foreign-born HMSM were safer when compared to US-born HMSM, $\chi^{2}(46, n=155)=$ 46, $p<.001)$. Findings from this study may be limited due to the small sample size. However, the major contribution of this study suggests that US-born HMSM may be at an increased risk for MDD and HIV infection from sexual risk behaviors (De Santis et al., 2009).

In a quantitative study designed to test a conceptual model that predicts the sexual behaviors of HMSM, De Santis et al. (2011) reported findings from a convenience sample of 100 HMSM, ranging in age between 18 and 51 years, who resided in South Florida. The conceptual model was based on the Web of Causation systems theory, which associates health and health risk conditions are interrelated with physical, psychological, and social factors. Six instruments were use to examine alcohol abuse (CAGE Questionnaire), body image (Adonis Complex Questionnaire), depression (CESD Scale), eating attitudes and behaviors (Eating Attitudes Test), self-esteem (RES Scale), and sexual behaviors (SSBQ). All tools were available in Spanish. Results support research studies that report mental health, expressed in terms of depression and selfesteem, are strong predictors of sexual behaviors among HMSM (De Santis et al., 2009). 
Furthermore, findings suggest that HMSM are at risk for a number of physical and psychological health conditions including: alcohol abuse (18\%), body image concerns (29\%), depression (25\%), risk for eating disorders (13\%), and low self esteem (12\%). Limitations of the study include using a convenience sample that was a cross sectional design, which may not truly be representative of the population of HMSM. The major contribution of this study is the findings, which include mental health as a significant predictor of sexual behaviors for HMSM.

In an effort to determine the correlates of depression in Hispanic men with HIV infection, De Santis et al. (2012) sampled 46 men in South Florida. The sample characteristics included 67.4\% ( $n=31)$ MSM, 30.4\% ( $n=14)$ heterosexual men, and $2.2 \%(n=1)$ who refused to report sexual orientation. The mean age for the sample was $44.20(S D=7.34)$, ranging from 26 to 56 years. Most of the participants were foreignborn $95.6 \%(n=44)$. The CES-D Scale was used to measure levels of depression among the participants and a score of 16 or greater indicated depression in this study. The Familism Scale was used to measure attitudes about family support and family membership. Scores for the Familism Scale range from 15 to 75, with higher scores indicating more perceived support. Self-esteem was measured using the RSES and scores equal or less than 16, indicated lower levels of self-esteem.

The Hispanic Stress Inventory (HIS) measured levels of stress for participants and higher scores indicated more stress. Substance abuse was measured using the Substance Abuse Behaviors Questionnaire (SABQ), which results in categorical data for past and current substance use. Violence was measured using the Violence Assessment questionnaire, which also results in categorical data. Negative correlations between 
depression and self-esteem was found to be significant ( $r=0.57, p<0.001)$, indicating higher levels of depression are associated with lower levels of self-esteem. Depression and Hispanic stress was also significant $(r=0.325, p<0.05)$, indicating higher levels of depression are associated with higher levels of Hispanic stress. Higher levels of depression were reported with higher levels of drunkenness and alcohol use as opposed to lower levels of depression ( $t=-2.41, p=0.023$ ), and higher levels of depression were reported with drug use in past 3 months $(t=-1.75, p=0.023)$. Higher levels of depression were associated with being intoxicated (from drugs or alcohol) before sexual activity in the past 3 months $(t=-3.25, p=0.003)$. Higher levels of depression were associated with experiences of physical abuse as an adult, $(\mathrm{t}=-2.25, p=0.030)$. Furthermore, the majority of the participants in the sample $(65 \% ; n=30)$ were depressed. A study limitation is the sample size. However, the study did examine the effect of depression on self-esteem, substance use/abuse and high risk sexual behaviors, and Hispanic men with HIV infection.

In a multi-city research study, Sandfort, Melendez, and Diaz (2007) surveyed a total of 912 HMSM, of which $72.2 \%$ were immigrants and $52.6 \%$ had resided in the US for 10 years or less. The sample’s participants’ mean age was 31.2 years, ranging between 20 to 40 years. Common reported experiences of homophobia during childhood were that $91 \%$ were told gays are not normal and $71 \%$ were told gays grow up to be alone. Additionally, $70 \%$ of the participants reported feelings that the respondent's homosexuality embarrassed their family and 29\% reported that they moved away from family or friends to live openly gay lives. Eighty percent of participants reported depressed moods, and 61\% reported sleep problems. Most seriously, 17\% had suicidal 
thoughts. This study's findings support that HMSM may be at risk for increased depression due to marginalization from family and cultural beliefs.

In a descriptive pilot study exploring the relationships among acculturation, depression, self-esteem, and substance abuse in Hispanic men, Vasquez, GonzalezGuarda, and De Santis (2011) recruited 164 Hispanic men in South Florida (82 heterosexual and $82 \mathrm{MSM})$. The mean age of participants was 41.25 years $(S D=9.03)$, ranging from 20 to 56 years. Participants were from 13 different countries, but the mean years reported for US residence was 18.72 years $(S D=13.05)$. The Bidimensional Acculturation Scale (BAS) contains two subscales (Hispanic and non-Hispanic subscale). BAS measures the acculturation level of the participants, in which higher scores indicate greater level of cultural activities for that particular subscale, i.e., a mean score of $\geq 2.5$ is indicative of biculturalism on either subscale. The CES-D Scale was used to measure levels of depression among the participants and a score of 16 or greater indicated depression in this study. Self-esteem was measured using the RSES and scores equal or less than 16, indicate lower levels of self-esteem. Substance abuse was measured using SABQ, which results in categorical data for past and current substance use. The variables that significantly predicted substance abuse were age $($ Wald $\chi 2=12.13, p<0.001)$ and depression (Wald $\chi 2=6.16, p=0.00$ ) (Vasquez et al., 2011). Participants who were older were less likely to report substance abuse in the past three months $(A O R=0.93$, $95 \% C I=0.89,0.97)$ and participants reporting increased depressive symptoms were more likely to report substance abuse $(A O R=1.05,95 \% C I=1.01,1.09)$. The findings from this study supports previous research findings linking depressive symptoms with substance use/abuse in Hispanic men (Gonzalez-Guarda, Florom-Smith, \& Thomas, 
2011; Gonzalez-Guarda et al., 2010; Maisto, Palfai, Vanable, Heath, \& Woolf-King, 2012). Additionally, this contradicts this study's proposed hypothesis that increased age in middle age HMSM may increase risk for depression; however, this study was not exclusively middle age HMSM. A study limitation is the small sample size of 82 HMSM, which is not representative of the HMSM community.

\section{Summary of Depressive Symptoms in the Literature}

From this review of the literature, only five studies were found on HMSM and depressive symptoms, but none were found with exclusively middle age HMSM. From these studies it can be concluded that HMSM may be at risk for depression, due to psychosocial issues, i.e., marginalization, low self-esteem, and substance use/abuse. Findings suggest that all of these psychosocial issues do not occur independently but may co-occur. Because some of the findings were non-significant with certain psychosocial issues, it still remains unclear of how many psychosocial issues equate to depression in HMSM, especially middle aged HMSM.

\section{Substance Use/Abuse}

In order to understand the substance use/abuse behaviors of middle age HMSM, a review of substance use/abuse in this population will be included in this study. Research has shown that substance use/abuse is associated with sexual risk (De Santis et al., 2009; Maisto et al, 2012). Because there is an abundance of literature on substance use/abuse behaviors for gay men and HMSM, it would be impossible to include all of the studies.

\section{Substance Use/Abuse in Men who have Sex with Men (MSM)}

In a study by Maisto et al. (2012) explored the effects of alcohol intoxication, sexual arousal, and condom attitudes on hypothesized determinants of safer sexual 
behavior among MSM. The sample included 117 MSM from the Boston and New York area with ages ranging from 21 to 50 years of primarily Caucasian men. The sample's total mean age and ethnic background for participants was not provided. Participants were randomly assigned to one of six conditions created by the combination of beverage condition (water control, placebo, or alcohol) and arousal (high sexual arousal or low, manipulated by viewing of erotic or non-erotic film clips). Determinants of safer sexual behavior were measured according to the information Motivation-Behavioral skills (IMB) model and hierarchical linear regression models were used for the exploratory analysis. It was hypothesized that both beverage condition and arousal would have direct effects on the dependent variables in the direction of greater sexual risk, but that beverage condition and arousal would interact such that individuals who were intoxicated and sexually aroused would show the highest degree of sexual risk. Direct effects of condom use attitudes were predicted in the direction of safer sex, and alcohol expectancies were predicted to interact with beverage condition, that is, expectancies would exert riskenhancing effects only in individuals who consumed alcohol. The results of both planned and exploratory analyses showed general support for the hypothesized enhancement of alcohol's effects on sexual risk behaviors. Limitations of the study include the ethnic makeup of MSM, who were primarily Caucasian. However, the results of this study support the effects of alcohol, sexual arousal, and determinants of risky sexual behavior.

In a secondary analysis from a RCT of 135 MSM, Well et al. (2011) investigated the efficacy of an intervention designed to reduce substance use and sexual risk behaviors. Participant characteristics included average age of $28.9(S D=7.23)$, with ages ranging from 18 to 65 . The ethnic composition for the sample included 39.26\% ( $n=53)$ 
Caucasian, 29.63\% ( $n=40)$ Hispanic, 20\% $(n=27)$, and African American 11.11\% $(n=$ 15). Additionally, only the men who reported any risky sex (UAI with casual or serodiscordant partner) during the preceding 30 days were included in the study. For the experimental design, analysis of variances (ANOVAs) were conducted in the study. The percentage of acts that occurred under the influence of substances had significant main effect of expectancies, $F(1,135)=15.74, P<0.001, \eta^{2}=0.108$, signifying higher sexual expectancies were associated with a higher percentage of acts using substances, i.e., under the influence. This main effect is qualified by a significant interaction between conflict and expectancies, $F(1,135)=4.19, P=0.043, \eta^{2}=0.031$ (Well et al., 2011). Post-hoc analyses reported that the high conflict/high expectancy participant group engaged in a significantly higher percentage of their sexual acts under the influence than the high conflict/low expectancy group $(P<0.01)$, and the low conflict/low expectancy group $(P=0.048)$, signifying participants high in conflict appear more sensitive to the effect of expectancies, compared to their low conflict counterparts (Well et al., 2011). Findings report a synergistic effect between conflict between motivation to practice safe sex and both a temptation to practice unprotected sex as well as the perceived benefits of unprotected sex. The study limitations include the small sample and its generalization to the population of MSM. The importance of this study is understanding the role of substance use in sexual behavior.

\section{Substance Use/Abuse in Hispanic Men who have Sex with Men (HMSM)}

Since substance use/abuse is a co-occurring issue for HMSM, the following two articles were reported earlier under social support for HMSM. Both of these articles also report substance use/abuse among HMSM. Fernandez et al. (2007) found that HMSM 
who were crystal methamphetamine users were more likely than non-drug users to have lower orientation to Hispanic culture (Hispanicism), higher attachment to the gay community, higher rates of UAI, and history of suicide attempts. The strength of this study was the multidimensional relationship between crystal methamphetamine use and high risk sexual behaviors among HMSM.

Fernandez et al. (2009) researched HMSM psychological factors associated with drug use and illustrated how risk and protective factors associated with drug use and high risk sexual behaviors guided development of Proyecto SOL. In the qualitative portion of their study, Fernandez et al. (2009) reported 75\% ( $n=15)$ of their sample used drugs and had high risk sexual behaviors. Additionally, $43 \%$ of the participants were classified as drug users (43\%) based upon their self-reported 6-month history of using the following drugs: cocaine, crystal methamphetamine, other amphetamines, poppers, ecstasy, GHB, Special K, and sildenafil in combination with other drugs. Drug users were more likely than non-drug users to be US born, interviewed in English, have higher monthly incomes, been previously tested for HIV infection, and tested seropositive for HIV infection (Fernandez et al., 2009). Drug users were more oriented to American culture (Americanism) and less Hispanicism than non-drug users (Fernandez et al., 2009). Drug users reported higher levels of loneliness, psychological distress, experiences of discrimination, more sex partners and higher rates of UAI, but less social connections (Fernandez et al., 2009).

In a community based participatory research (CBPR) partnership in North Carolina to establish prevalence estimates of risk behaviors among immigrant Latino MSM living in their rural area, Rhodes et al. (2012) recruited 190 Latino MSM. The 
sample consisted of $80 \%$ Mexican MSM having an average age of 25.5 years.

Respondent driven sampling (RDS) was used to identify, recruit, and enroll Latino MSM to participate in an interviewer administered behavioral assessment. RDS is an extension of chain referral methods. The CBPR partnership developed the assessment iteratively based on formative studies and literature review; in addition, they used validated Spanish language scales when available. Findings included: daily smoking 6.5\%, past 30 day heavy episodic drinking $35.0 \%$, past 12 month marijuana use 56.0\%, cocaine use $27.1 \%$, past 3 month prevalence estimates of sex with at least one woman $21.2 \%$, multiple male partners $88.9 \%$, and inconsistent condom use $54.1 \%$. A limitation of the study includes not addressing the years of residence in the US. Providing information on health risk behaviors of Latino MSM immigrants is the strength of this study.

De Santis et al. (2014) reported findings from a qualitative grounded theory approach study in South Florida, which included HMSM $(n=20)$. Sample characteristics and study variables was reported earlier under loneliness in the HMSM population. Themes regarding negation (denial) of sexual risk, substance abuse as a buffer, and the burden of violence saturated the authors' findings and substance abuse was found as a means to buffer negative events or emotions, such as loneliness, separation from family (due to migration or marginalization), and loss of social support for HMSM (De Santis et al., 2014). Findings from this study indicate that a lack of psychosocial support for HMSM resulted in high risk sex, substance abuse, and violence, consequentially increasing risk for HIV infection.

In a descriptive, comparative design study by De Santis, Valdes, ProvencioVasquez, Patsdaughter, and Gattamorta (2014) data was collected from 164 Hispanic 
men in South Florida (77 heterosexual and 87 MSM) to compare the substance use behaviors of Hispanic men by sexual orientation. Participants mean age for the entire group was reported as 41.25 ( $S D=9.03$, range $18-55$ years). Monitoring the Future Survey by Bachman, O'Malley, and Johnston (1979) was the classical instrument utilized to capture substance use and abuse behaviors. The 35-question instrument included numerous filtering or contingency questions with responses of “yes," "no," and "refuse to answer." If the participant selected "yes” then the questions would include the frequency of substance use among lifetime, previous 3-months, and monthly prevalence. A significant relationship between sexual orientation and substance use, whereby the heterosexual group reported more substance use than the HMSM group, was found to be statistically significant, $\left[X^{2}(160, N=1)=7.38, p<.007\right]$. After controlling for income, education, religion, HMSM were half as likely to report drug use as compared to their heterosexual counterparts, $(O R=0.484, p=0.51)$ (De Santis et al., 2014). This finding contradicts previous research findings and provides new information on substance use among HMSM in South Florida. A limitation of this study is the small sample size and the snowball sampling strategy used to recruit participants.

\section{Summary of Substance Use/Abuse in the Literature}

Even though Hispanic men in the US do not experience disproportionate rates of substance use and abuse when compared to other ethnic groups, they are disproportionately affected by the consequences of substance use and abuse due to these disparities (Gonzalez-Guarda et al., 2010; Substance Abuse and Mental Health Service Administration [SAMHSA], 2010). An abundance of research with HMSM demonstrated a consistent association between alcohol and substance use and high risk 
sexual practices; however, an apparent gap in the literature was found with few studies found to be exclusively on HMSM. Research has shown that substance use lowers inhibitions, enhances sexual pleasure, and decreases condom usage (Well et al., 2011). Therefore, exploring whether substance use/abuse is a co-occurring issue for middle age HMSM warrants further investigation.

\section{Sexual Behaviors}

In order to understand the sexual behaviors of middle age HMSM, a review of sexual behaviors in this population will be included in this study. Of all the variables in this study, sexual behaviors of HMSM generated the most research studies. Since there is an abundance of literature on sexual behaviors for gay men and HMSM, it would be impossible to include all of the studies.

\section{Sexual Behaviors in Men who have Sex with Men (MSM)}

In a quasi-experimental design in New York City metropolitan area, Balan et al. (2013) recruited 89 ethnically diverse (27\% Caucasian, 25\% Hispanic, 21\% AfricanAmerican) participants with a mean age of 32, of which $98 \%$ self-identified as gay. The in-depth qualitative interview inquired about participants' definition of bareback sex, feelings toward and experiences of bareback sex, concerns about HIV infection, substance use, and ethnic and racial identity (Balan et al., 2013). Information about HIV testing practices and HIV status were collected using Computer Assisted Self Interview (CASI) software. Balan et al. (2013) found concerns about acquiring HIV infection among MSM who participated in bareback sex. Interestingly, participants mentioned strategies to reduce the risk of HIV infection, such as avoiding HIV infected sex partners, 
decreasing the number of partners with whom they had bareback sex, and avoiding the exchange of bodily fluids (Balan et al., 2013).

Kelly et al. (2010) reported findings from a study funded by a grant from the National Institute of Mental Health to characterize sexual risk practices and identify factors predicting levels of sexual risk or safety in an ethnically and racially diverse sample of gay or bisexual men living in Milwaukee, Wisconsin. The researchers identified venues that MSM frequent and recruited using an index recruitment visit. The index recruitment visit involved researchers observing and unobtrusively seeking to identify the individual from MSM groups who appeared to be the center of positive social attention received from and directed to others in their circle. These individuals identified are considered the index or "seed." Indexes were informed of the study and asked to help identify and recruit participation of their groups of friends. Seventy five indexes collectively provided the names of 418 potential network members, with each index network ranging in size from three to ten people. After removing duplication and participants who did not meet the inclusion criteria, the sample size for analysis was 215 men.

Participants were interviewed using audio computer administered self-interviews (ACASI) modalities in private 60- to 90-minute sessions based on AIDS-Related Psychosocial Scales. Among the 215 men, 61 (28\%) were African American, 32 (15\%) were Latino, 114 (53\%) were White, and 8 (4\%) were of other races or ethnicities; furthermore, participants’ mean age was 29 years and White and African American networks were composed primarily of men of the same race. Ninety-five percent of men ( $n=204$ ) reported having same-sex activity during the past year. The large majority of 
men $(84 \%, n=180)$ described themselves as mainly or exclusively homosexual.

Twenty-eight percent ( $n=17$ ) of African American men defined themselves as bisexual, mainly heterosexual, or exclusively heterosexual relative to $11 \%(n=13)$ of White and $15 \%(n=5)$ of Latino men. The large majority of men in the sample $(95 \%, n=201)$ reported having had an HIV test. Thirty eight percent of participants had an STI in their lifetime, with a past STI significantly more common among African American (48\%) than either White (38\%) or Latino (22\%) MSM. Overall, 26\% of men said that they had received money or valuables in exchange for sex in their lifetime, $14 \%$ in the past year. During the past year, 26\% of African American and 22\% of Latino MSM said they received money or valuables for sex relative to 7\% of White MSM, a significantly lower percentage. Over $70 \%$ of men reported recent casual sexual partners. About one fourth of men engaged in UAI with a casual partner or with multiple partners in the past 3 months.

Limitations include measuring egocentric social networks and not assessing the extent to which social network members may have also been sexual partners with one another. Additionally, this study was carried out in a single Midwestern city, whereas, cities with larger or smaller gay communities may have different MSM networks. However, the major contribution of this study illustrates that the social network to which a man belonged, weaker risk reduction intentions, and greater substance use independently predicted a range of high risk sexual behaviors. Additionally, there were modest but significant correlations between the risk related characteristics of network members and network leaders; therefore, interventions directed toward social networks of MSM have the potential to strengthen safer behavior norms within a persons' immediate social environment, target HIV prevention to community segments at greatest risk, and 
reach persons who are hidden and do not appear in visible gay community venues but can be accessed through their social connections with others in the community.

\section{Sexual Behaviors in Hispanic Men who have Sex with Men (HMSM)}

Lo et al.’s (2011) study examined factors associated with communication about condom use and UAI in a US sample of immigrant HMSM. Two sets of hypotheses were tested. The first hypothesis for this study was whether Hispanic cultural beliefs were associated with a lower likelihood of communication about condom use during participants' most recent sexual encounter. Second, the authors examined whether communication about condoms predicted a greater likelihood of reported condom use.

The sample included 356 HMSM from the New York metropolitan area ranging from 20 to 70 years of age, with a mean of 36 years (Lo et al., 2011). Logistic Regression was used to determine which of the three variables of culture, partner influence, or physical status had on the report of UAI after communication regarding condom use during the most recent sexual encounter. Step 1 in the Logistic Regression model used cultural variables (participant's country of birth and the Latino Beliefs

Inventory score) which resulted in non-significance $\chi^{2}(3, n=356)=5.59, p=.13$. In the second step, partner variables (whether last sexual encounter was a main partner and was partner seroconcordant) were added and resulted in significance $\chi^{2}(5, n=356)=24.19, p$ $<0.05$. The third step in the Logistic Regression model added physical variables (participant's level of sexual desire at the time of the sexual encounter and whether the respondent was intoxicated with alcohol or recreational drugs during the sexual encounter) which also resulted in a significant change $\chi^{2}(7, n=356)=35.60, p<$ 0.0001 . 
Participants who reported higher levels of sexual desire and intoxication were significantly less likely to have communicated about condom use (Lo et al., 2011). Although partner and physical variables added significant explanatory power for predicting communication about condom use beyond the cultural variable set, seroconcordance on its own was not significant. Interestingly, one of the cultural factors (Columbian nationality) that did not provide explanatory power in the model for predicting communication about condom use, was the strongest predictor on its own, recording an odds ratio $(O R)$ of 1.24, signifying that Columbian MSM were 1.24 times more likely to engage in condom negotiation with their sexual partners.

In the second Logistic Regression model, Lo et al. (2011) added another variable, communication about condom use, to the aforementioned variables (cultural, partner, and physical) to predict the likelihood of condom use during participants' most recent sexual encounter. Cultural variables were used in Step 1 in the Logistic Regression model and again resulted in non-significance $\chi^{2}(3, n=356)=2.72, p>.05$. The second step added partner variables which resulted in significance $\chi^{2}(5, n=356)=24.64, p<.05$. The third step in the model added physical variables, which also resulted in a significant change $\chi^{2}(7, n=356)=31.74, p<0.0001$. The fourth and final step in the model added participant's communication about condom use during last sexual activity, resulting in significance $\chi^{2}(8, n=356)=93.27, p<0.0001$. Similar to the first model, the cultural variables did not predict condom use during the participant's most recent sexual encounter. Additionally, a lower likelihood of condom use was found when the partner was a main partner, when the partner was seroconcordant, and the participant was intoxicated. Noting an $O R$ of 9.31, the strongest predictor of condom use were 
participants who communicated with each other about condom use; therefore, they were nine times more likely to use condoms than those who did not communicate (Lo et al., 2011). This emphasizes the importance of encouraging communication about condom use in HMSM populations.

In a qualitative study involving $20 \mathrm{HMSM}$, ranging in age from 26 to 54 years, and their perceived reasons for high risk sexual behaviors within the context of primary relationships, the authors found relationships among high risk sex, substance use, and violence among the participants studied (De Santis et al., 2012). The following eight categories were reported as factors that influence high risk sexual behaviors and subsequent HIV infection risk among HMSM in primary relationships: drugs and alcohol; sex outside the relationship; concealment of HIV infection; violence; sexual violence; consensual sexual violence; absent family support; and relationship of sex, substances and violence (De Santis et al., 2012). These links support the limited scientific knowledge for HMSM in primary relationships and their associated high risk behaviors that predispose them to HIV infection.

In a RCT designed to develop an intervention to decrease high risk sexual behaviors among HMSM, Carballo-Dieguez et al. (2005) recruited 180 participants, with ages ranging from 18 to 67 years, and equally divided them into either an experimental or control group. The intervention session themes were based on the empowerment theory and used factors identified in prior research as determinants of unsafe sex, which covered oppression, transgression of rules, excuses (or rationalizations), substance use, goal setting, the role of pleasure, self-efficacy and plans for the future (Carballo-Dieguez et al., 2005). The study design included four repeated measures: one baseline evaluation 
before the intervention and three follow-up assessments scheduled at 2-, 8- and 14months (Carballo-Dieguez et al., 2005). Attrition rates were as follows: $87 \%$ attended the 2-month follow up and 85\% of the participants attended both the 8- and 14-month follow up, but budget constraints terminated the study at 14 -month follow up when $64 \%$ of the participants had been interviewed. The Sexual Practices Assessment Schedule for MSM was adapted (used a Likert scale instead of an absolute number) for this study. This instrument measures the number of occasions of different sexual practices (manual/ oral/anal/vaginal; insertive/receptive; protected/unprotected) over the two months prior to the interview with three different types of male and female partners. Other variables measured were serostatus and serostatus disclosure, attendance at HIV education and counseling services, intentions to use condoms, pleasure experienced with and without condoms, substance use, childhood sexual experiences and gender role. Study results indicated changes in sexual behavior after baseline assessment. At the 2-month follow up, $46 \%$ of the men in the experimental group and $54 \%$ of those in the control group reported no UAI during the previous two months. At the 8-month follow up, 44\% of men in the intervention group and $40 \%$ of those in the control group reported no UAI. At the last follow up (14-months), $51 \%$ of the men in the intervention group and $46 \%$ of those in the control group reported no UAI. The experimental group had higher levels of self efficacy with intentions to participate in safer sexual behaviors post intervention. Despite reduction in UAI in the experimental group, and an increase in UAI in the control group, the results were not statistically significant.

O’Donnell, Stueve, Joseph, and Flores (2014) adapted an intervention named VOICES/VOCES $(V / V)$, which is a brief single-session group (with 4 to 9 men per group) 
intervention that focuses on sexual safety, condom use and partner negotiation, for use with culturally diverse HMSM to test the efficacy in reducing high risk sexual behaviors and to promote HIV testing. Each $V / V$ single-session is 45 -minutes long, followed by a 15-20 minute discussion, and ended with a 10-minute educational lecture on condoms. As part of a CDC multisite initiative, $V / V$ was adapted to the existing No Excuses/Sin Buscar Excusas study to meet the prevention needs of English and Spanish speaking HMSM.

In a randomized trial, O’Donnell et al. (2014) recruited 370 HMSM, of which 180 participants were in the control group and 190 participants were in the experimental group. The mean age of the control group was 36 years and self-identified as gay (57.5\%) and bisexual (34.1\%). The mean age of the experimental group was 37 years and identified as gay (70 \%) or bisexual (26.8 \%). Sexual behaviors were measured at baseline and at a 3-month follow up. Participants answered questions regarding frequency of UAI, number of sexual partners, and gender of sexual partners for the last three months.

Additionally, information on self-efficacy with condom usage and HIV infection testing during the 3-month follow up was also collected. At baseline, participants reported the following sexual behaviors in the previous three months: having an average of 5.4 male partners, 6.5 total partners (any gender), 57.9\% had a main partner, engaged in an average of 7.4 acts UAI, 60.8\% reported not using condom during last sexual act, and $65.4 \%$ had ever been tested for HIV infection. Post intervention, the experimental group showed a decrease in UAI occasions from 8.4 to 3.8, and the number of UAI acts post intervention revealed a statistical significance $(F=4.10, p<.05)$. Logistic 
regression examining condom use at last sexual encounter revealed that men post intervention were 1.5 times more likely to report condom usage (protective behavior), $A O R=1.69 ; 95 \% C I 1.02-2.81, p<.05)$. The authors analyzed intervention effects for older and younger men (40 years and under).

In the subsample of younger men $(n=208)$ all of the following were significant $(p<0.05)$ for the intervention group: condom use at last sexual encounter, no condom use with last two partners, and number of UAI occasions. Younger men post intervention reported more positive condom attitudes than their control counterparts (AOR 2.07; 95 \% CI 1.02-4.22, $p<0.05)$. Young men in the control group were twice as likely to report they had not used condoms with their last two partners (27.1\% compared to $14.8 \%$, $p<0.05)$. Additionally, testing for HIV infection was significant for younger men post intervention (46.2 \% compared to $20 \%$ in the control group, $p<0.05$ ). In summary, compared to the control group, HMSM who participated in the $V / V$ intervention reported significantly fewer unprotected sex acts and were more likely to use condoms at their last sex encounter (O’Donnell et al., 2014).

In a descriptive, comparative cross-sectional study, De Santis et al. (2015) collected data from 163 Hispanic men in South Florida (80 heterosexual and 83 MSM) to compare sexual behaviors by sexual orientation. Participants mean age was reported as $39.86(S D=9.74)$ for the heterosexual group and mean age $42.76(S D=8.00)$ for the HMSM group. Age of sexual debut and number of sexual partners did not result in a significant difference. However, differences were noted in the numbers of men from each group who engaged in anal sex, i.e., 21 heterosexual men (26.25 \%) engaged in anal sex as compared to 72 HMSM (86.75 \%). Furthermore, 16 (76.19 \%) of the 21 
heterosexual men who reported engaging in anal sex reported UAI, and of the 72 MSM who engaged in anal sex, only 20 (27.78 \%) reported UAI, resulting in a statistical significant difference [ $\left.X^{2}(2, N=72)=17.04, p<.001\right]$ (De Santis et al., 2015). Therefore, HMSM who engaged in anal sex were more likely to use a condom during anal sex, when compared to the heterosexual men. This finding contradicts previous research findings and provides new information on sexual practices among HMSM in South Florida. A limitation of this study is the small sample size and the snowball sampling strategy used to recruit participants.

\section{Summary of Sexual Behaviors in the Literature}

Research with HMSM has referenced high rates of HIV infection from their sexual risk behaviors (De Santis et al., 2012; Lo et al., 2011). There exists vast knowledge on MSM and high risk sexual behaviors. Even though some limitations did exist in the articles found in the literature review, an apparent gap of knowledge was found regarding HMSM, particularly middle aged, and all their associated high risk behaviors.

\section{Summary of Chapter 2}

The importance of preventing these high risk sexual behaviors, which can result in HIV infection, can increase the likelihood of healthy aging among this population and consequently decrease their health disparity. Referenced high rates of HIV infection associated with HMSM warrant the need for research with this population. Limitations did exist in the articles found in the literature review, an apparent gap of knowledge was found regarding HMSM, particularly middle aged, and their associated high risk sexual behaviors. 
This literature review found a large amount of research related to high risk sexual behaviors for HMSM; however, no research was found exclusively on middle age HMSM. The research available discussed unique characteristics and experiences that affect subsequent high risk sexual behaviors, such as engaging in UAI, drug and alcohol use/abuse. Many of the studies revealed several co-occurring disorders (lack of social support, loneliness, depressive symptoms, and substance use) that precipitate engagement in high risk sexual behaviors. The importance of preventing these high risk sexual behaviors, which can result in HIV infection, may address some health disparities across the nation for HMSM.

As stated earlier, population projections estimate the Hispanic population to double by 2050, while the 65 year and older population steadily increases faster than younger groups, and estimates of the LGBT community are 9 million; therefore, an emergence of concern for understanding middle aged HMSM's predisposition to high risk sexual behaviors that can lead to STIs and HIV infection is of utmost concern (US Census, 2011). As the population becomes more diverse, there exists an urgency to address minority groups less likely studied in the past, such as the middle aged HMSM, who experience psychosocial issues, substance use/abuse, and high risk sexual practices that ultimately increase HIV infection risk. 


\section{CHAPTER 3-METHODOLOGY}

\section{Research Design}

The methodological approach that was utilized for this study is a nonexperimental, cross-sectional research method. Non-experimental research can be either descriptive or correlational. A descriptive study can describe and organize variables or characteristics of the sample without examining the relationships between the variables or attempt to infer/predict the variables under investigation in order to provide information on behaviors, attitudes, or other characteristics of a particular group (Salkind, 2013). Correlational research measures the degree (strength and nature) of the relationship between two variables (Salkind, 2013).

Correlation coefficients are generalities, i.e., it describes the entire group, not every individual in the sample. Correlational designs do not imply causation but focus on describing relationships between variables. Since correlational designs explore the strength of the relationship between two continuous variables, it is able to indicate whether the relationship is positive (as one variable increases, so does the other) or negative (as one variable increases, the other decreases). Furthermore, other statistical tests that will be employed in data analysis for this study are Logistic Regressions, twoway, between-groups analysis of variances (two-way ANOVAs), and Multiple Regression.

Logistic Regression is used to predict an outcome that is categorical from predictor variables that are continuous and/or categorical (Salkind, 2013). For instance, comparing different conditions (e.g. social support, loneliness, depressive symptoms) on a variety of outcome measures (e.g. drug/alcohol use, sexual behaviors, HIV infection 
status). The two-way ANOVA design compares the mean differences between groups that have been split on two independent variables. A two-way ANOVA allows researchers to understand if there is the possibility of an interaction effect between the two independent variables on the dependent variable (Salkind, 2013). For instance, this researcher can understand if there is an interaction between partner status and religiosity on levels of social support amongst middle-aged HMSM; hence, partner status (partnered or not) and religiosity (religious affiliation or not) are the independent variables and social support is the dependent variable. Multiple regression allows the researcher to learn more about the relationship between several independent variables and a dependent variable (Salkind, 2013). This allows the researcher to evaluate the importance of each of the independent variables in the model and test the overall fit of the model with the data collected.

\section{Independent or Predictor Variables}

The independent or predictor variable refers to the intervention or treatment that may be manipulated (Salkind, 2013). The independent variable can be evaluated with respect as to how it is correlated and compared to the dependent variable. In this study, the independent variables were loneliness, depressive symptoms, substance use/abuse, and social support.

\section{Dependent or Outcome Variables}

The dependent or outcome variable is the main characteristic being measured in the study (Salkind, 2013). The dependent variable is the principal variable of interest in the study and may be affected by the independent variable. In this study, the dependent variable was sexual behaviors. 


\section{Attribute Variables}

Attribute or demographic variables are pre-existing characteristics that describe the sample in the study. The attribute variables in this study included categorical and continuous variables. The continuous data included age, years of residence in the US, offspring (if any), and number of years of education. Categorical data included relationship status, living arrangements, nationality, self-identified sexual orientation, religion, employment status, income, health insurance status, and perceived health status.

\section{Sample}

As demographics change, the US has become a rapidly growing multicultural population. Hispanics account for more than half of the growth in the total population between 2000 and 2010, making them the nation's largest ethnic minority (CDC, 2011). According to the US Census Bureau (2011), it is estimated that 52 million Hispanics reside in the US, representing approximately $16.7 \%$ of the US total population. It is projected that the Hispanic population will reach 132.8 million by 2050 (30\% of the US population).

\section{Setting}

South Florida has a large population of Hispanics (US Census, 2011). Since South Florida has a large gay community, the chosen setting was Miami-Dade County. The Florida Department of Health in Miami-Dade County (2010) estimates that 1 in 97 Hispanic men in Miami-Dade County is living with HIV infection. The July 2009 census reported that Miami-Dade County included a population of 2,500,625 of which $48.3 \%$ are male and 61.4\% are Hispanic; furthermore, reported male non-family households included 280,292 with a median age of 35.6 years (City-Data, 2012). For the purpose of 
this study, Hispanic gay and bisexual men were grouped into the category of HMSM. According to Lieb et al. (2006), there are 1,455,490 Hispanic men in Florida of which 4 to $10 \%$ are MSM. Therefore, the HMSM population of Florida is estimated to be between 58,220 to 145,549 .

Increased recruitment of middle age HMSM participants was noted after identifying venues that middle age HMSM frequent and placing flyers in each location. Following the Institutional Review Board (IRB) approval from Florida International University, flyers promoting the study were posted at multiple sites across South Florida including organizations, bars, barbershops, immigration offices, local stores and street fairs, where middle age HMSM are known to congregate. There are numerous gay bars in Miami-Dade County that operate seven days a week with ranging working hours. Prior to placing flyers and utilizing these establishments and venues, this researcher obtained verbal consent from the owners or managers of these establishments.

\section{Selection of Participants}

To accurately represent the population of interest in this study, the selection of participants is vital. Because it is impossible to locate and survey the entire middle age HMSM population of Miami-Dade county, a representative subset with a certain number of participants from the middle age HMSM population was obtained as the sample for this study. Additionally, a non-probability sampling technique of convenience sampling was utilized.

According to Burns and Grove (2009), convenience sampling is considered a weak sampling method, since it is not random; however, convenience sampling is the most common approach used in nursing research studies. Using convenience sampling 
does not ensure that the derived sample is representative of the larger population. Despite the weaknesses of convenience sampling, it is less expensive, less time consuming, and useful in this proposed study, whereby the researcher can readily access a sample of middle age HMSM.

The sample size is calculated after the study’s significance level, effect size, and power are determined. The alpha or significance level is typically set at 0.05 . Alpha is the probability of rejecting a true null hypothesis and making a Type I error. Type I errors infer reporting that there is a finding when indeed there is not. Since the alpha for this study was set at 0.05 , there is a five percent chance of committing a Type I error (Polit \& Beck, 2012).

The study's power refers to the probability of rejecting the null hypothesis, thereby avoiding a Type II error. Type II errors infer reporting that there is not a finding when indeed there is a finding. Since a power of $80 \%$ is generally considered acceptable for research studies, this was selected for this study (Polit \& Beck, 2012).

Effect size is a proposed measure that explains the magnitude of the difference between groups, but does not take into account the variability in scores (Sullivan \& Feinn, 2012). According to Cohen (1987), effect sizes are classified as small $(d=0.2)$, medium $(d=0.5)$, and large $(d \geq 0.8)$, in which a medium effect of 0.5 is visible to a vigilant observer. Even if the statistical analysis is significant, if the two study groups' means do not differ by at least 0.2 standard deviations, the difference is minor (Sullivan \& Feinn, 2012).

The researcher may estimate the effect size if they are unsure of the associations of the variables under investigation (Cohen, 1987). Since effect size is the main finding 
of a quantitative study and this study employed Multiple Regression as its most powerful statistical method, a medium effect size of 0.5 was chosen (MacCallum, Browne, \& Cai, 2006). However, the researcher realized that this designation does not take into account the accuracy of the instruments and the diversity of the study population, instead provides a general guide. Lastly, according to desired significance level of 0.05 and the power of 0.80, the sample size should be no less than 150 participants for an estimated effect size of 0.5 (moderate difference effect) (Polit \& Beck, 2012). Since there resulted a wide range of possible responses, some variables were collapsed and converted to dichotomous variables; however, this will be discussed in Chapter 4.

\section{Inclusion Criteria}

Inclusion criteria help the researcher define and identify the characteristics needed to answer the research questions. Inclusion criteria for participation in this study included: (a) self-identification as a Hispanic or Latino man; (b) self-identification as gay/homosexual or bisexual; (c) 40 to 65 years of age (middle aged); (d) required to be able to listen or read and comprehend English or Spanish; and (e) currently reside in South Florida before enrolling in the study.

\section{Exclusion Criteria}

Exclusion criteria help the researcher identify characteristics of the population that are not desired in the study. Participants may have certain conditions that may limit participation. Exclusion criteria in this study included participants who: (a) refuse to selfidentify as Hispanic or Latino men; (b) do not self-identify as MSM; (c) are not 40 to 65

years of age; (d) cannot comprehend English or Spanish; and (e) do not reside in MiamiDade county. 


\section{Instrumentation}

Data on middle aged HMSM's attitudes and behaviors were collected using five instruments. The following are the instruments that were used in this study: Multidimensional Scale of Perceived Social Support (MSPSS), the University of California, Los Angeles (UCLA) Loneliness Scale, the Behavioral Risk Assessment Tool (BRAT), the Center for Epidemiologic Studies Depression (CES-D) Scale, and a standard demographic questionnaire. The paper packet with the five instruments was collated into a booklet entitled “Hispanic Men’s Health Study.”

\section{Demographic Questionnaire}

A demographic questionnaire was used to collect both categorical and continuous data. The continuous data included age, years of residence in the US, number of offspring (if any), and number of years of education. Categorical data included relationship status, living arrangements, nationality, self-identified sexual orientation, religion, employment status, income, health insurance status, and perceived health status. A faculty member from the University of Miami School of Nursing \& Health Studies translated the demographic questions professionally and it was made available to this researcher for use in this study.

\section{Multidimensional Scale of Perceived Support (MSPSS) Scale}

The variable of social support was measured using the MSPSS instrument. The MSPSS instrument is designed to measure perceptions of support for an individual from three sources: family, friends, and a significant other (Zimet et al., 1988). The MSPSS scale was designed as a research instrument that incorporates ease of use and participant time conservation. 


\section{Description of the MSPSS Scale}

The MSPSS scale is a 12-item instrument with four items for each subscale (family, friends, and significant other). The items that Zimet et al. (1988) included in the MSPSS scale were developed from research literature on social support, which identified an inverse relationship of social support with anxiety and depression. Since the MSPSS scale is easy to use, conserves time, and has a strong foundation in research, it has been widely used as the instrument to measure social support in research studies where social support is a variable under investigation. The MSPSS scale can be completed in approximately 5-minutes and requires approximately 2-minutes to score. Since the MSPSS is in the public domain in both English and Spanish versions, it does not require permission for use.

\section{Norms of the MSPSS Scale}

The MSPSS scale was normed with 275 undergraduate university students (mean age of 18.6, $S D=.88$ ) of which 136 were female and 139 were male. From this 275 undergraduate sample, 69 participated in test-retest procedures during instrument development. Additionally, Liu and Mustanski (2012) utilized the MSPSS instrument with 248 lesbian, gay, bisexual and transgender (LGBT) youth (47.7\% male) with a mean age of 18.76 years $(S D=1.34)$ and with $11.4 \%$ Hispanic participants. The MSPSS demonstrated excellent internal consistency for family (Cronbach's $\alpha=.90$ ) and peer support (Cronbach's $\alpha=.91)$.

\section{Scoring of the MSPSS Scale}

The MSPSS scale can be scored for each subscale individually or used as the total scale score. The total score measures the overall support of an individual. There are four 
items in each subscale (family, friends, and significant other) that have to be tallied then divided by four to result in a subscale score. The total scale score is calculated by adding all of the items then dividing them by 12. Items are rated on a 7-point Likert-scale ranging from 1 (very strongly disagree) to 7 (very strongly agree). Zimet et al. (1988) suggest that a mean scale score ranging from 1 to 2.9 could be considered low support; a score of 3 to 5 could be considered moderate support; and a score from 5.1 to 7 could be considered high support.

\section{Reliability Data on the MSPSS Scale}

Numerous studies have shown the MSPSS to have good internal and test-retest reliability, good validity, and a stable factorial structure (Mustanski \& Lui, 2012). The MSPSS is a summation instrument with Cronbach’s alphas ranging from .85 to .91 for each subscale and the reliability of the total scale was .88 (Zimet et al., 1988). High internal consistency was found with test-retest reliability over 2- to 3-month interval ranging from .72 to .85 for the subscales, and .85 reliability for the total scale (Zimet et al., 1988).

\section{Validity of the MSPSS Scale}

The MSPSS scale was found to have excellent concurrent validity. Since Zimet et al. (1988) hypothesized that a lack of perceived social support would relate to anxiety and depression, the subscales for depression and anxiety in the Hopkins Symptoms Checklist (HSCL) were used to construct validity. Zimet et al. (1988) reported correlations between the MSPSS scale and the HSCL supported the following prediction, which constructed validity: perceived support from family was significantly inversely related to depression $(r=-.24, p<.01)$ and anxiety $(r=-.18, p<.01)$; perceived support from 
friends was significantly inversely related only to depression $(r=-.24, p<.01)$; perceived support from a significant other was significantly inversely related only to depression $(r=$ $-.13, p<.05)$; and the summated perceived total support scale was significantly inversely related only to depression $(r=-.25, p<.01)$.

\section{Spanish Version of the MSPSS Scale}

According to Marin and Marin (1991), research with bilingual Hispanic populations has shown that participants prefer to respond to questionnaires in Spanish. Therefore, the Spanish version of the instrument was utilized. Fortunately, the MSPSS scale was translated into Spanish and is available in the public domain (American Academy of Pediatrics, 2010).

\section{University of California, Los Angeles (UCLA) Loneliness Scale}

The variable of loneliness was measured using the UCLA Loneliness scale, version three. The UCLA Loneliness scale is designed to measure self-reports of concurrent loneliness as well as feelings of social isolation (Russell et al., 1978). The UCLA Loneliness scale was designed as a research instrument that has been widely used with MSM, including HMSM (Grov, Golub, Parsons, Brennan, \& Karpiak, 2010; Martin \& Knox, 1997; Sandfort et al., 2007).

\section{Description of the UCLA Loneliness Scale}

The UCLA Loneliness scale is a 20-item instrument measuring an individual's subjective feelings of loneliness and social isolation. Russell et al. (1978) developed the items on the scale from an in-depth review of other loneliness scales and research literature on both loneliness and social isolation. Currently, the UCLA Loneliness scale is in its third version, which is the instrument that was utilized in this study. The UCLA 
Loneliness scale is easy to use and has a strong foundation in research. It has been widely used as the instrument to measure loneliness in research studies where loneliness is a variable under investigation (Grov et al., 2010; Martin \& Knox, 1997; Sandfort et al., 2007). The UCLA Loneliness scale can be completed in approximately 8-minutes and requires approximately 3-minutes to score. Since the UCLA Loneliness scale is in the public domain in both English and Spanish versions, it does not require permission for use.

\section{Norms of the UCLA Loneliness Scale}

As part of a larger study investigating loneliness, the UCLA Loneliness scale was normed with 492 university students, 227 from UCLA (76 males and 151 females) and 265 from Tulsa University (130 males and 135 females). One-hundred and two student volunteers from Tulsa University participated in a two month test-retest procedure during instrument development. In a multi-city research study with self-identified HMSM, Sandfort et al. (2007) reported an internal consistency of 0.78 for the adapted UCLA Loneliness scale with 302 HMSM from Miami, 309 HMSM from New York and 301 HMSM from California. Additionally, Hubach et al. (2015) studied factors of loneliness, HIV infection related stigma, and condom use in a sample of 100 HIV infected MSM residing in rural Indiana. The variable of loneliness was assessed using the revised third version of the UCLA Loneliness scale. Composite measures for loneliness using the UCLA Loneliness scale resulted in Cronbach's $\alpha=0.941$ (Hubach et al., 2015).

\section{Scoring of the UCLA Loneliness Scale}

The UCLA Loneliness scale contains 20 items, which is scored using a 4-point letter scale. The following are the ranges for the 4-point letter scale responses: $\mathrm{O}$ 
signifies "I often feel this way", S signifies "I sometimes feel this way"), R signifies "I rarely feel this way, and $\mathrm{N}$ signifies "I never feel this way." Instructions indicate to score all of the letters $\mathrm{O}$ and $\mathrm{N}$ as zero, $\mathrm{R}$ as one and $\mathrm{S}$ as two, suggesting that higher total scale scores indicate lower self ratings of satisfaction and happiness with increased loneliness (Russell et al., 1978).

\section{Reliability Data on the UCLA Loneliness Scale}

Numerous studies have shown the UCLA Loneliness scale to have good internal and test-retest reliability, good validity, and a stable factorial structure (Russell et al., 1978; Russell, Peplau, \& Cutrona, 1980; Russell, 1980; Weeks, Michela, Peplau, \& Bragg, 1980). The UCLA Loneliness scale is a summation instrument with a Cronbach alpha of .96 for the first version and ranged from .89 to .94 in its third version (Russell et al., 1978; Russell et al., 1980). The third version of the UCLA Loneliness scale was tested using 301 elderly participants (over 65 years of age), of which 121 were male and 180 were females. With this elderly sample, the UCLA Loneliness scale was readministered 12 months later and resulted with a high internal consistency and test-retest reliability correlations scores equal to .73, which signified a similar finding found with prior psychometrical testing (Russell et al., 1980).

\section{Validity of the UCLA Loneliness Scale}

The UCLA Loneliness scale was found to have concurrent validity. Several validity criteria were used to examine the UCLA Loneliness scale. First, Russell et al. (1978) compared results among groups (clinic versus student scores), which resulted ( $r$ $(45)=.79, p<.001)$ for the self-report question about current loneliness and the loneliness scale. Since loneliness has been linked to other emotional states, such as 
depression and anxiety, the researchers significantly correlated their findings with the Beck Depression scale (1967) and its measures of depression $(r(131)=.49, p<.001)$ and anxiety $(r(131)=.35, p<.001)$. Lastly, Russell et al. (1978) significantly correlated their findings with the anxiety subscale from the Multiple Affect Adjective Checklist by Zuckerman and Lubin (1965) resulting in $(r(65)=43, p<.01)$. Therefore, the UCLA Loneliness scale supports its theoretical framework by linking loneliness to emotional states such as depression, anxiety, feelings of boredom and emptiness.

\section{Spanish Version of the UCLA Loneliness Scale}

Research employing the Spanish version of the UCLA Loneliness scale has reported good reliability and validity with a stable factor analysis. For instance, Borges, Prieto, Ricchetti, Hernandez-Jorge, and Rodriguez-Naveiras (2008) administered the Spanish version of the UCLA Loneliness scale to 522 undergraduate university Spanish and Italian students, whose median age was 19.6, and reported that the scale had a theoretical and analytical coherent bifactorial structure with good reliability and validity. Therefore, the Spanish version of the UCLA Loneliness scale was utilized in this study. The UCLA Loneliness scale is available in the public domain in Spanish (Borges et al., 2008).

\section{Behavioral Risk Assessment Tool (BRAT)}

Developed collaborative from a CDC funded five state and 5-year project (Prevention With HIV-Infected Persons Project [PHIPP]), the BRAT was designed to collect HIV risk behaviors, including sexual and drug/alcohol behaviors, homelessness, incarceration, and HIV testing history (Wisconsin HIV Prevention Evaluation Work 
Group, 2000). Both sexual and drug/alcohol related behaviors were measured using the BRAT. In this study, this instrument was the most informational and time-consuming.

\section{Description of the BRAT}

The BRAT is a 61-item instrument with categorical and a few continuous variables, i.e., number of sexual partners by gender; years with main partner if applicable; and the number of times and how many drug, alcohol and/or tobacco used in the past 30 days. The BRAT can be completed in approximately 30 minutes and does not yield a score. BRAT measures sexual behaviors for the previous 3-month period and drug, alcohol and/or tobacco usage in the past 30 days. The sexual behavior items include: number of partners by gender, types of sexual acts, frequency of condom use, number of partners with which the participant had unprotected anal or vaginal sex, and serodiscordant or discordant sexual acts (Wisconsin HIV Prevention Evaluation Work Group, 2000). The drug, alcohol, and tobacco measure items include questions pertaining to the frequency of drug use (types of injectable and non-injectable drugs) for the previous 30-day period and includes if needles were new, used and/or shared (Gasiorowicz et al., 2005).

\section{Norms of the BRAT}

The BRAT was not intended to be a research tool, but a descriptive clinical tool, which provides an idea of individuals' HIV infection risk behaviors. BRAT has a strong foundation from prior research and was developed as a risk assessment instrument to improve HIV infection testing and counseling services (Chen, Branson, Ballenger, \& Peterman, 1998). The BRAT was utilized with 362 participants, of which $16 \%$ were 
Hispanic, 37\% self-identified as MSM, 96\% were HIV infected, and 79\% were over 30 years of age (Gasiorowicz et al., 2005).

Several adapted versions of the BRAT have been successfully utilized in research studies (Berkley-Patton, Moore, Hawes, Thompson, \& Bohn, 2012; Hawes \& BerkleyPatton, 2014; Gasiorowicz et al., 2005). In 2014, Hawes and Berkley-Patton employed the BRAT with a 297 sample of African American church and community participants, of which $69.4 \%$ were female, $83.9 \%$ were heterosexual and had a mean age of 46.3 (SD =13.9). Furthermore, the English version of the BRAT is in the public domain, which indicates that it does not require permission for use.

\section{Scoring of the BRAT}

In its originality, the BRAT was never intended to yield a score (Wisconsin HIV Prevention Evaluation Work Group, 2000). However, adapted versions of the BRAT developed scoring parameters (Berkley-Patton et al., 2012; Hawes \& Berkley-Patton, 2014). For this study, the BRAT was used in crosstabs to compare the variables by yes or no. The days of drug, alcohol, and tobacco use yielded a continuous outcome measure.

\section{Reliability Data on the BRAT}

As stated earlier, the BRAT was intended to be used as a screening tool and does not yield a score; therefore, reliability testing for the original BRAT is not available. No previous level of internal consistency has been published (Hawes \& Berkley-Patton, 2014). Additionally, Hawes and Berkley-Patton (2014) did not report their findings for reliability in the adapted version of the BRAT. Test-retest was not performed using the adapted version of the BRAT, but a Cronbach alpha of .75 was reported illustrating that 
the internal consistency of the adapted BRAT and deemed acceptable (Hawes \& BerkleyPatton, 2014).

\section{Validity of the BRAT}

There is no reported concurrent validity for the BRAT. Hawes and BerkleyPatton (2014) did not report validity of the adapted BRAT used in their research study. Even though the BRAT does not have any validity reported, it is a valuable clinical instrument, which provides information or an idea of an individual's HIV infection risk.

\section{Spanish Version of the BRAT}

The Spanish version of the BRAT was professionally translated and made available to this researcher by the University of Miami School of Nursing \& Health Sciences.

\section{Center for Epidemiologic Studies Depression (CES-D) Scale}

The variable of depression was measured using the Center for Epidemiologic Studies Depression (CES-D) scale. The CES-D instrument was designed to measure depressive symptomology in the general population (Radloff, 1977). There have been considerable findings to support the use of the CES-D Scale with the HMSM population; however, none of these studies had exclusively middle age HMSM (De Santis et al., 2012; De Santis et al., 2012; De Santis et al., 2009).

\section{Description of the CES-D Scale}

The CES-D Scale is a 20-item instrument with components that include:

depressed mood, feelings of guilt and worthlessness, feelings of helplessness and hopelessness, psychomotor retardation, loss of appetite and sleep disturbance (Radloff, 1977). The items in the CES-D Scale were developed from research literature on 
depression and other depression scales. Since the CES-D Scale is easy to use, conserves time, and has a strong foundation in research, it has been widely used as the instrument to measure depression in research studies where depression is a variable under investigation. The CES-D Scale can be completed in approximately 4-minutes and requires approximately 2-minutes to score. Since the CES-D Scale is in the public domain in both English and Spanish versions, it does not require permission for use.

\section{Norms of the CES-D Scale}

The CES-D Scale was normed with a total of 3,835 female and male Caucasian participants (18 years and older) from Missouri and Maryland. Test-retest procedures were performed on 1,971 of these participants during instrument development. Additionally, participants demonstrated a strong to moderate internal consistency over a 2- to 8-week period (correlations ranging from .51 to .67) and a weak to moderate internal consistency over a 3-month to 1-year period (correlations ranging from .32 to .54) (Radloff, 1977).

\section{Scoring of the CES-D Scale}

The CES-D Scale is a 20-item Likert-scale summation instrument with scores ranging from 0 to 60, with higher scores indicating more depressive symptoms (Radloff, 1977). To break tendencies toward responses, four items were worded in the positive direction and require reverse scoring. Items are rated on a Likert-scale ranging from 0 (rarely or none of the time) to 3 (most or all the time). Radloff (1977) did not suggest an exact score that signified depressive symptoms, but most studies indicate a score above 16 to be indicative of depression; therefore, this study employed a score above 16 as the score considered to represent depressive symptoms. 


\section{Reliability Data on the CES-D Scale}

The CES-D Scale reported high internal consistency with Cronbach’s alpha .85 in the general population. During instrument development, Radloff (1977) also sampled 70 female and male psychiatric patients as another means for norming the instrument, Cronbach’s alpha of.90 was reported, indicating high internal consistency. This high internal consistency may signify response bias (answering all responses in the same direction), but to diminish this bias Radloff (1977) positively worded items 4, 8, 12, and 16.

\section{Validity of the CES-D Scale}

The CES-D Scale was found to have excellent concurrent validity. From a review of the literature, Radloff (1977) constructed validity using several depression and mood scales. The CES-D Scale discriminates strongly between patient and general population groups for depressive symptomology and has been found to correlate well with other scales designed to measure depression (Radloff, 1977).

\section{Spanish Version of the CES-D Scale}

The Spanish version of the CES-D was utilized for this study. Fortunately, the CES-D Scale was translated into Spanish and is available in the public domain.

\section{Field Procedures}

Following Institutional Review Board (IRB) approval from Florida International University, flyers promoting the study were posted at multiple sites across Miami-Dade county including organizations, bars, barbershops, immigration offices, local stores and street fairs, where HMSM are known to congregate/frequent. Flyers included a contact 
telephone number for interested participants with future locations and times that this researcher would collect data.

There are numerous gay bars in Miami-Dade County that operate seven days a week with ranging working hours. Identifying venues that middle age HMSM frequent and placing flyers in each location increased recruitment of middle age HMSM participants. Prior to placing flyers and utilizing these establishments and venues, this researcher obtained verbal consent from the owners or managers of these establishments. After obtaining verbal consent from the owners and managers of these establishments, data collection dates and times were negotiated, as well as the physical space required for reasonably private data collection to take place.

On the negotiated dates for data collection, the principal investigator (PI) set up a table in a private setting, such as a designated private area (private table at a bar/restaurant). Both English and Spanish versions of the five instruments collated into the booklet entitled “Hispanic Men’s Health Study” was made available with pens and clipboards.

In an attempt to recruit participants, this researcher approached potential participants and invited them to participate in the study. Regardless of participation, the potential participants were thanked for their time. Potential participants were screened by the PI for inclusion criteria. If the participant met the inclusion criteria and agreed to participate, the participant was provided a written consent in their desired language, English or Spanish, explaining the study. If the participant agreed to the consent, this researcher verbally obtained informed consent for enrollment. 
Since there are no personal identifying information, the middle age HMSM who met inclusion criteria and were willing to participate were provided a clipboard with the collated paper packet including the five instruments and a pen, and made available in the participant's preferred language. For those participants who were unable to read or those who liked the questionnaires read due to vision difficulties, the Spanish speaking PI, who is female and familiar with the population, read the survey questionnaires in their preferred language of English or Spanish.

As much privacy as can be afforded from the study site was provided to answer and complete the research questionnaires. The PI was available for any questions or problems, and an estimate of 47-minutes to an hour was needed to complete the questionnaires. Once the five instruments were completed, the researcher skimmed through the questionnaires to ensure that all answers had a response (or were not answered on purpose). The PI then asked participants to place their booklet in a covered locked box to maintain confidentiality. The PI was the only person to have access to this locked box and completed surveys remained in this locked box until data entry. The participants were thanked and $\$ 10.00$ cash stipend was provided for study participation. Additionally, once questionnaires were complete, participants were encouraged to refer other middle age HMSM from their social networks (snowball sampling).

\section{Data Collection \& Recording}

Five self-administered questionnaires assisted in collecting data. Since the researcher was on site during data collection, clarification for items in questionnaires was provided. Questionnaires included fixed-alternative (multiple-choice) and open-ended 
questions. Participants were provided as much time as needed to complete questionnaires, but were expected to approximately take 45 minutes for completion.

After data were collected, each booklet was assigned a number by date collected for data entry. This number was used as a reference to a particular survey in case the researcher or dissertation committee seeks clarification for a particular response. The Statistical Package for the Social Sciences (SPSS) 22.0 was used for data analysis.

\section{Protection of Human Subjects}

Florida International University (FIU) promotes Responsible Conduct of Research (RCR) practicing high standards of ethics and accountability in planning, implementation, behavior and information dissemination. Students, faculty and staff are encouraged to provide safe work environments, which foster the values of a shared responsible community. The Responsible Conduct of Research training is required for

all undergraduates, graduate students, and postdoctoral researchers who will be supported by National Science Foundation (NSF) to conduct research for a period of more than 120 days.

This study was reviewed and approved by FIU’s Institutional Review Board prior to submission. The protection of participants began with recruitment procedures. Advertisements and flyers included: the PI's name and telephone, the purpose of this study, inclusion criteria, and incentive for participation.

Since quantitative results were viewed in aggregate format and no responses were linked to an individual participant, confidentiality was ensured and signed informed consent foregone. This study may have involved some discomfort for the participant, which included the following: 
- A potential risk associated with the study is disclosure of sexual risk behavior that puts others at risk; however, no identifying information was collected. The PI answered any safer sex questions that the participants had and provided them with an informational flyer listing HIV infection or AIDS support service agencies, community based organizations, and healthcare providers.

- They may become upset by questions, which ask about experiences with discrimination.

The investigator considered the records confidential to the extent permitted by law. The participant's records may be reviewed for audit purposes by authorized FIU personnel, who will be bound by the same provisions of confidentiality. Procedures to protect the participant's privacy included the following:

- No identifying information was recorded on the screening form or questionnaires.

- Electronic data was stored in the researcher's password-protected computer and hard copy forms were stored in locked file cabinets at the researcher's home.

- Any publications or presentations of results from this study will present group findings and will not be connected to any individual in the study. No benefit was promised to the participants from this study. The study is expected to benefit gay and bisexual men who have a predisposition to HIV infection. It will help healthcare providers such as nurses and doctors better understand the influence of age, 
loneliness, substance use, depression, and social support on sexual behaviors, so as to provide better care and improve health outcomes among middle age HMSM.

\section{Summary of Chapter 3}

This study is the first step for developing interventions targeted to reduce HIV infection among middle age HMSM. This study is essential in providing data to direct programs of health education geared to decrease high risk sexual practices among middle age HMSM, which will subsequently reduce morbidity and mortality of this high risk

group. Data from this study will be valuable to clinicians, educators and those who make public policy decisions. 


\section{CHAPTER 4-RESULTS}

\section{Introduction}

The purpose of this study was to investigate the effects of age, loneliness, substance use, depression, and social support on high risk sexual behaviors that predispose middle aged HMSM to HIV infection risk. Direct logistic regression was performed to assess the impact of factors (age, social support, loneliness, depression, and substance use/abuse) on high risk sexual behaviors. A two-way between-groups analysis of variance (ANOVA) was conducted to explore the impact of partner status and religion on social support, levels of loneliness, depressive symptoms, substance use/abuse, and sexual risk. Social support was measured using the MSPSS Scale, loneliness was measured using the UCLA Loneliness Scale, depressive symptoms was measured using the CES-D Scale, and substance use/abuse and high risk sexual behaviors was measured using the BRAT. Correlations between study variables were noted. The two-tailed Pearson product-moment correlation coefficient $(r)$ was used to test directions of relationships and strength among the study variables. Multiple Regression was performed to assess the association of a number of factors on the likelihood that respondents would report engaging in high risk sexual behaviors.

Data were collected from HMSM who were 40 to 65 years of age, living in an urban area in South Florida. A non-probability sampling technique of convenience sampling was utilized to recruit participants at various community sites such as organizations, bars, barbershops, local stores and street fairs, where middle age HMSM were available to be sampled. Data were collected over a 5-month period using a selfreport questionnaire, which consisted of four standardized instruments, and a 
demographic questionnaire. Data were analyzed using Statistical Package for Social Sciences (SPSS) 22.0 for Macintosh.

\section{Description of the Sample}

Convenience sampling resulted in a total of 150 questionnaires collected from members of this targeted population. All 150 questionnaires were included in data analysis, because all of the questionnaires met the aforementioned inclusion criteria discussed in Chapter III. Post-hoc power analysis using G*POWER software (Faul, Erdfelder, Buchner, \& Lang, 2009; Faul, Erdfelder, Lang, \& Buchner, 2007) was conducted in the same manner as discussed in Chapter III using the established parameters. For the study's most powerful statistical test of Multiple Regression with five related variables, an alpha of .05 and a sample of 150 participants, an actual power of 0.9764 for a medium effect size, and an actual power of 0.9994 for a large effects size was noted. Therefore, this indicates that the study sample size of 150 should be adequate to note associations between the study's variables.

\section{Demographic Characteristics of the Sample}

The sample size of this study was 150 participants. All participants reported their age. Table 1 summarizes the descriptive statistics of the variable of age.

Table 1.

Age of the Study's Participants $(n=150)$

\begin{tabular}{llll}
\hline$M$ & $S D$ & Median & Range \\
45.54 years & 4.71 & 44.00 & $20(40-60)$ \\
\hline
\end{tabular}

All 150 participants self-identified as Hispanic and reported country of origin. Table 2 summarizes the variables of country of origin. Table 3 summarizes the variables of birth, 
length of time in the US, relationship status, and religiosity. Participants were asked to report socioeconomic information, which included employment status, highest level of education, and monthly income in US dollars (Table 4).

Table 2.

Country of Origin $(n=150)$

\begin{tabular}{lcc}
\hline Country & Frequency $(n)$ & Percentage \\
\hline Cuba & 52 & $34.7 \%$ \\
USA & 21 & $14 \%$ \\
Colombia & 16 & $10.7 \%$ \\
Venezuela & 10 & $6.7 \%$ \\
Argentina & 9 & $6 \%$ \\
Peru & 8 & $5.3 \%$ \\
Puerto Rico & 7 & $4.7 \%$ \\
Dominican Republic & 6 & $4 \%$ \\
Guatemala & 5 & $3.3 \%$ \\
Mexico & 3 & $2 \%$ \\
Chile & 2 & $1.3 \%$ \\
El Salvador & 2 & $1.3 \%$ \\
Nicaragua & 2 & $1.3 \%$ \\
Panama & 2 & $1.3 \%$ \\
Ecuador & 1 & $0.7 \%$ \\
Honduras & 1 & $0.7 \%$ \\
Other (Canadian-Spaniard) & 1 & $0.7 \%$ \\
Paraguay & 1 & $0.7 \%$ \\
Uruguay & 1 & $0.7 \%$ \\
\hline
\end{tabular}


Table 3.

Birth, Length of Time in the US, Relationship Status, and Religiosity $(n=150)$

\begin{tabular}{ll}
\hline Variable & Descriptive Statistics (n and \%) \\
\hline Birth & $21 ; 14 \%$ \\
USA & $129 ; 86 \%$ \\
Foreign-born & \\
\hline Length of Time in the US & 18.09 \\
$M$ & 15.97 \\
$S D$ & 11.50 \\
Median & $57.0(1-58)$ \\
Range & \\
\hline Relationship Status & $56 ; 37.3 \%$ \\
Partnered & $94 ; 62.7 \%$ \\
Not Partnered & \\
\hline Religiosity & $124 ; 82.7 \%$ \\
Religious & $26 ; 17.3 \%$ \\
Not Religious &
\end{tabular}


Table 4.

Socioeconomic variables of the Participants $(n=150)$

\begin{tabular}{ll}
\hline Variable & Descriptive Statistics ( $N$ and \%) \\
\hline Employment Status & \\
Employed, Yes & $137 ; 91.3 \%$ \\
Not employed, No & $13 ; 8.7 \%$ \\
\hline Monthly Income in US dollars ${ }^{a}$ & \\
\$0 to \$499 & $1 ; 0.7 \%$ \\
\$500 to \$999 & $6 ; 4 \%$ \\
\$1000 to \$1999 & $28 ; 18.7 \%$ \\
\$2000 to \$2999 & $33 ; 22 \%$ \\
\$3000 to \$3999 & $22 ; 14.7 \%$ \\
\$4000 to \$4999 & $20 ; 13.3 \%$ \\
\$5000 to \$5999 & $10 ; 6.7 \%$ \\
\$6000 or above & $7 ; 4.7 \%$ \\
\hline Educational Level & \\
Less than a High School Education & $n=25 ; 16.7 \%$ \\
Completed High School & $n=52 ; 34.6 \%$ \\
Associate’s Degree & $n=42 ; 28 \%$ \\
Bachelor's Degree & $n=17 ; 11.3 \%$ \\
Master's Degree & $n=10 ; 6.7 \%$ \\
Doctoral Degree & $n=4 ; 2.7 \%$ \\
\hline
\end{tabular}

Some data are missing: ${ }^{a} n=127$

\section{Exploratory Data Analyses for Measurement of Variables}

Histograms and frequency distributions with the normal distribution curves were conducted for all measurement variables to determine outliers, skewness, kurtosis, and to note any missing values. The skewness value is an indication of the symmetry of the distribution (Salkind, 2013). The variables of the MSPSS Scale and the BRAT were 
normally distributed or near-normally distributed. The UCLA Scale and the CES-D Scale were found to be positively skewed. According to Pallant (2010), many of the scales and measures in the social sciences have scores that are positively or negatively skewed due to the underlying nature of the construct; therefore, this does not necessarily mean that there is a problem with the data. For instance, clinical measures of depressive symptoms are often positively skewed in the general population, with most individuals reporting few symptoms of this condition. Total scores for the MSPSS Scale, UCLA Scale, and the BRAT were computed as a continuous score. The MSPSS Scale contained three subscales; however, the hypotheses did not require the use of specific subscales, only the total scale score.

It is difficult to determine how extreme either skewness or the kurtosis values must be before they indicate a problem for the assumption of normality; however, the standard error for both the skewness and kurtosis can be calculated in SPSS by dividing either score by its standard error (Pallant, 2010). If the result is greater than \pm 1.96 , it suggests that your data are not normal with respect to that statistic. Applying this rule of thumb of dividing each value by its standard error results in the following: 0.78 for skewness and 0.08 for kurtosis for the UCLA Scale, 0.79 for skewness and 0.87 for kurtosis for the MSPSS Scale, 1.06 for skewness and 0.36 for kurtosis for the CES-D Scale, and 0.70 for skewness and 1.02 for kurtosis for the BRAT which are all well within \pm 1.96 limits, suggesting that the departure from normality is not too extreme (Darlington, 1990; Pallant, 2010). Figures 1, 2, 3, and 4 represent the histograms with superimposed normal distribution curves for reported loneliness, depressive symptoms, social support, and high risk sexual behaviors. 
Figure 1.

Histogram of the Total Scores: Social Support

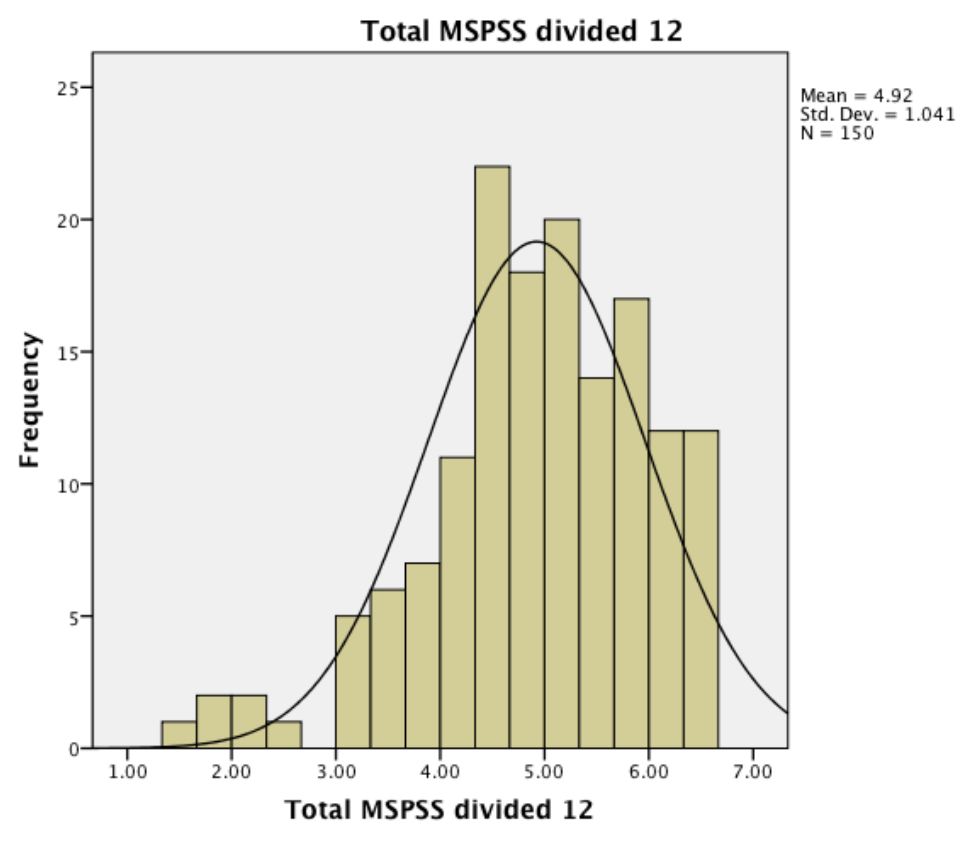

Figure 2.

Histogram of the Total Scores: Reported Loneliness

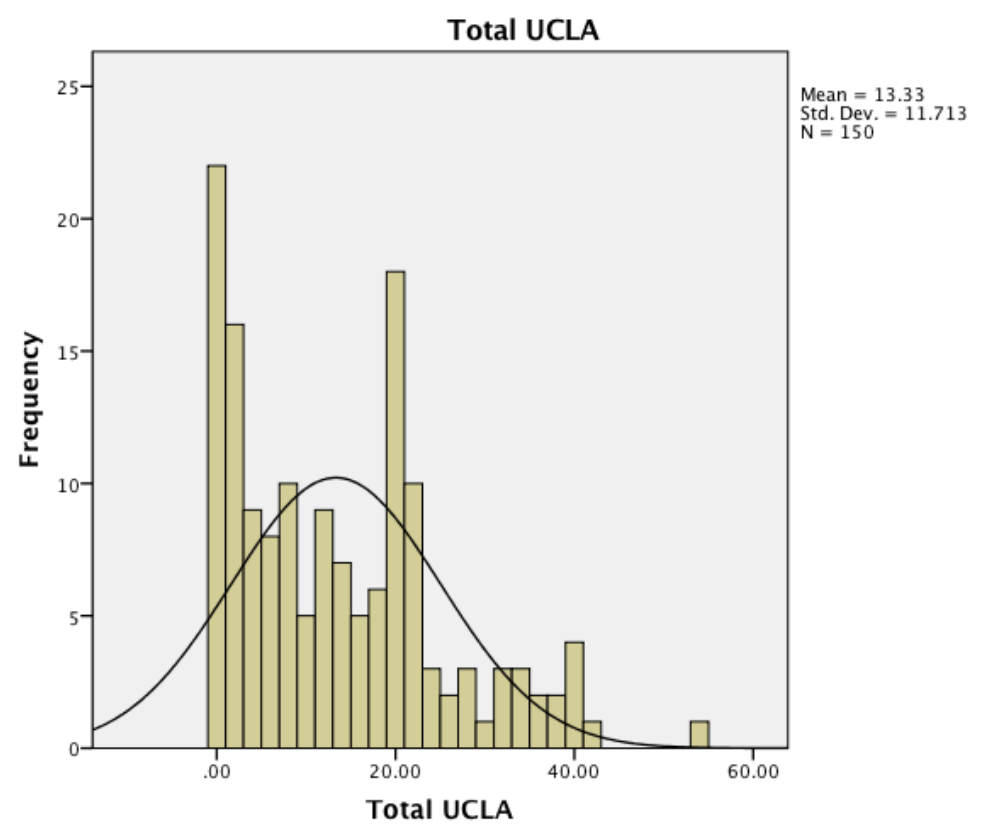


Figure 3.

Histogram of the Total Scores: High Risk Sexual Behaviors

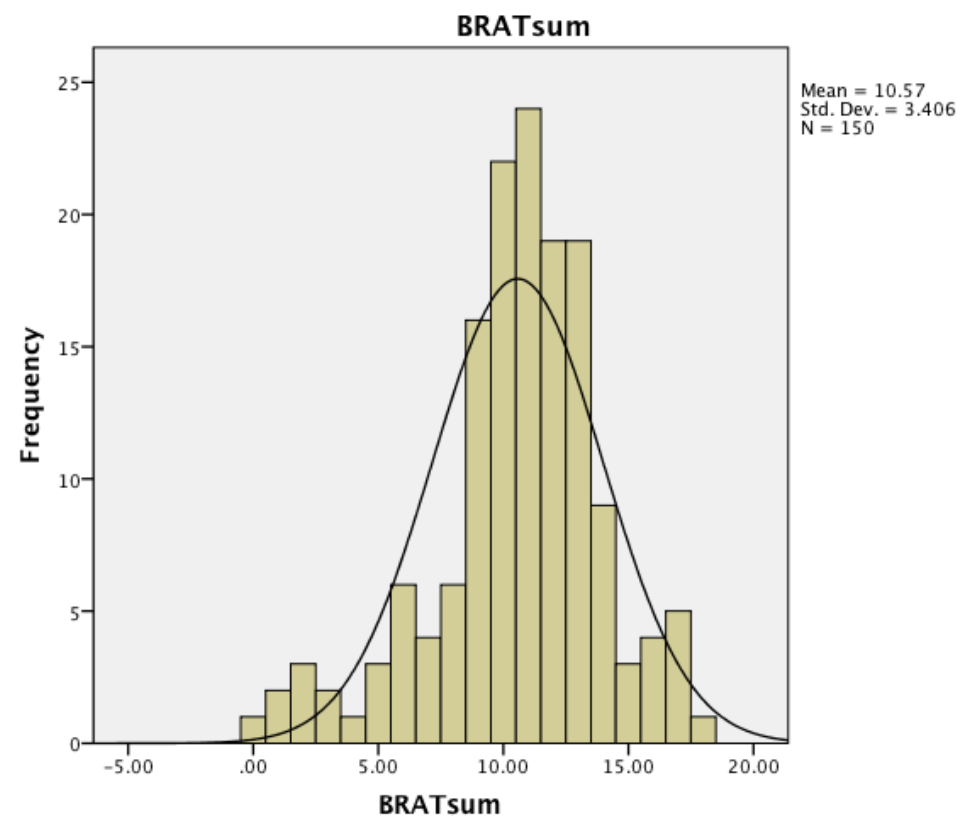

Figure 4.

Histogram of the Total Scores: Depressive Symptoms

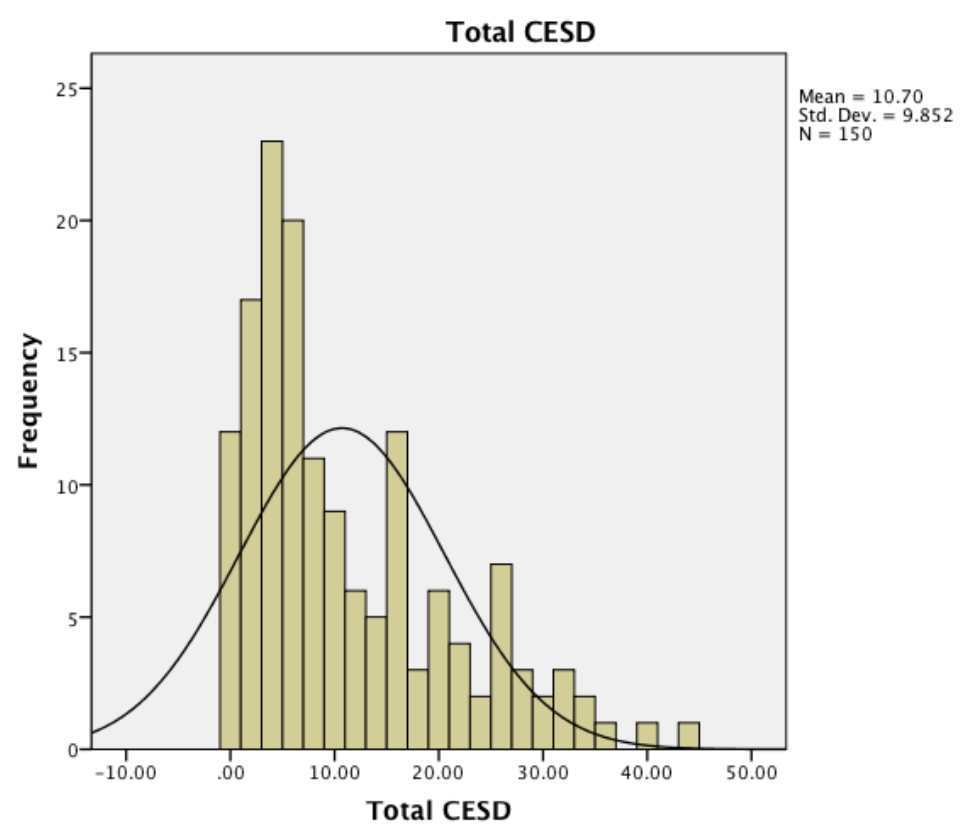




\section{Measurement Assessments}

\section{Multidimensional Scale of Perceived Support (MSPSS) Scale}

The MSPSS Scale was used to measure social support from three sources (family, friends and a significant other). This instrument consists of 12 items rated on a 7-point Likert-scale ranging from 1 (very strongly disagree) to 7 (very strongly agree), with four items for each subscale (family, friends, and significant other). The MSPSS scale can be scored for each subscale individually or used as a total scale score. The total score measures the overall support available to an individual. The total scale score is calculated by adding all of the items then dividing by 12 . Scores range from 1 to 7 , with scores ranging from 1 to 2.9 considered low support, scores of 3 to 5 considered moderate support, and scores of 5.1 to 7 considered high support (Zimet et al., 1988). A histogram with the superimposed normal distribution curve for the total score of this variable revealed a relatively normal distribution of scores.

In this study, the MSPSS Scale was used to measure the overall social support of an individual. High reliability estimates were demonstrated in this study of urban men for this scale. The internal consistency (Cronbach's alpha) for the total scale was 0.92. Additionally, Cronbach’s alphas for each subscale were explored and resulted in .90 for the significant other subscale, .95 for the family subscale, and .92 for the friends subscale. This estimate is consistent with Zimet et al.'s (1988) reported alphas ranging from .85 to .91 for the subscales and .88 for the total scale in the general population.

\section{University of California, Los Angeles (UCLA) Loneliness Scale}

The UCLA Loneliness scale was used to measure subjective feelings of loneliness and social isolation among the participants. This instrument consists of 20 items rated on 
a scale of 0 (I never feel this way) to 3 (I often feel this way). Scores are totaled and ranged from 0 to 60 , with higher scores indicating lower self-ratings of satisfaction and happiness with increased loneliness (Russell et al., 1978). A histogram with the superimposed normal distribution curve for the total score of this variable revealed that the distribution of scores were positively skewed. Since most people in the general population are not lonely, this is an expected finding for this construct (Pallant, 2010).

The UCLA Scale was used to measure an individual's subjective feelings of loneliness and social isolation in this study. High reliability estimates were demonstrated in this study of urban men for this scale. The internal consistency (Cronbach's alpha) for the total scale was 0.96. This estimate is consistent with both Russell et al.'s (1978) and Russell et al.’s (1980) reported alphas ranging from .89 to .94 for the general population.

\section{Behavioral Risk Assessment Tool (BRAT)}

The BRAT was used to collect risk behaviors, including sexual and drug/alcohol behaviors, homelessness, incarceration, and HIV testing history. This instrument consists of 61 items scored using categorical and continuous variables (Wisconsin HIV Prevention Evaluation Work Group, 2000). This data included the number of sexual partners by gender, years with main partner if applicable, and the number of times and how many drugs, alcohol and/or tobacco were used in the past 30 days. For analyses, the BRAT was divided into two total scores, sexual risk and alcohol/drug use. Twenty-eight questions had responses that included yes to no and refused. These questions were scored as 1 for yes, 0 for no or refused. Additionally, five questions regarding condom use included responses ranging from never to always, and not applicable. These five questions were recoded as 0 for responses that were not applicable or never, and 1 for 
always, usually, sometimes, and occasionally. The BRAT total score measured sexual risk and was calculated by adding the 28 questions with the 5 recoded questions, with sum scores ranging from 0 to 32; furthermore, higher scores indicated higher levels of sexual risk behaviors. The second total score was alcohol/drug use, i.e., if any drugs, alcohol and/or tobacco were used in the past 30 days. The scores was dichotomized, 0 for no, refused or not applicable, and 1 for yes. The scores ranged from 0 to 1 , with a score of 1 indicating substance use (drugs, alcohol and/or tobacco). Five questions regarding the number of sexual partners by gender and the years with main partner were excluded from the totaled BRAT sum and alcohol/drug sum because the continuous scores could not be dichotomized. A histogram with the superimposed normal distribution curve for the total score of this variable revealed a relatively normal distribution of scores.

The BRAT was used to measure HIV risk behaviors, including sexual and drug/alcohol behaviors, homelessness, incarceration, and HIV testing history of an individual in this study. However, high reliability estimates were not demonstrated in this study of urban men with this tool. The internal consistency (Cronbach's alpha) for the total scale (sexual risk and alcohol/drug use) was 0.84. Additionally, Cronbach's alphas for each subscale were explored and resulted in .75 for the sexual risk subscale, and .47 for the alcohol/drug use subscale. This estimate is relatively consistent with the only found reported alpha from Hawes and Berkley-Patton (2014) of .75 for the total scale in the general population. 


\section{Center for Epidemiologic Studies Depression (CES-D) Scale}

The CES-D Scale was used to measure depressive symptoms among the participants. The instrument consists of 20 items rated on a scale of 0 (rarely or none of the time or less than 1 day) to 3 (most or all the time or 5 to 7 days), with four items that are negatively scored $(4,8,12$, and 16$)$. The four items are reversed scored, and then all 20 items are totaled to signify each individual's total score of depressive symptoms. Scores range from 0 to 60, with scores that are greater than 16 indicating higher levels of depressive symptoms (Radloff, 1977). High reliability estimates were demonstrated in this study of urban men for this scale. The internal consistency (Cronbach's alpha) for the total scale was 0.92. This estimate is consistent with Radloff's (1977) reported alpha of .90 for the general population. A histogram with the superimposed normal distribution curve for the total score of this variable revealed that the distribution of scores was positively skewed. Since most people in the general population do not experience depressive symptoms, this is an expected finding for this construct (Pallant, 2010).

\section{Descriptive Findings of the Study Variables}

\section{Multidimensional Scale of Perceived Support (MSPSS) Scale}

The MSPSS Scale consists of 12 items rated on a 7-point Likert-scale ranging

from 1 to 7, with four items for each subscale (family, friends, and significant other). Higher scores indicate greater available support for an individual. In this study, this instrument was used as a total scale score, which measures the overall support available to an individual. Scores ranging from 1 to 2.9 are considered low support, scores of 3 to 5 are considered moderate support and scores of 5.1 to 7 are considered high support 
(Zimet et al., 1988). The total MSPSS scores for this study ranged from 1.6 to 6.5 ( $M=$ 4.92, $S D=1.04)$.

The mean of 4.92 noted in this study is lower than that noted by Zimet et al. (1988). The reported mean from Zimet et al. (1988) was $5.80(S D=.86)$, indicating high support. However, the study sample was college students who were predominantly Caucasian. Although this study was a non-Caucasian study, the results of this study are different from Zimet et al. (1988) indicating that this sample of the HMSM population may have less available support than the general population.

\section{University of California, Los Angeles (UCLA) Loneliness Scale}

The UCLA Loneliness scale consists of 20 items rated on a scale of 0 to 3 , with total scores ranging from 0 to 60 . Higher scores indicate lower self-ratings of satisfaction and happiness with increased loneliness (Russell et al., 1978). The total UCLA Loneliness Scale scores ranged from 0 to $53,(M=13.33, S D=11.71)$. These results indicate that the mean in this study has relatively high levels of satisfaction and happiness with lower levels of loneliness. Since there are no demarcated scores to indicate lower versus higher levels of loneliness, it is necessary to determine the demarcation point between lower and higher levels of loneliness. The point established as the demarcation point was the mean, $M=13.33$. Any total scores less or equal to 13 will be interpreted as higher self-ratings of satisfaction and happiness with decreased loneliness, while scores greater than 13 will signify lower self-ratings of satisfaction and happiness with increased loneliness.

In 1996, Russell reported the results from psychometric analyses from four prior studies using the UCLA Loneliness Scale. Study samples included a large $(N=489)$ 
predominately Caucasian college student population, a registered nurse population $(N=$ $310)$, a teacher population ( $N=316$ ), and participants who were over 65 years old, an elderly population $(N=301)$. The student sample scores ranged from 20 to $74,(M=$ $40.08, S D=9.50)$. The nurses sample scores ranged from 20 to $75,(M=40.14, S D=$ 9.52). The teacher sample scores ranged from 10 to 37 , $(M=19.22$, $S D=5.11)$. The elderly sample scores ranged from 20 to $59,(M=31.51, S D=6.92)$. Additionally, significant mean differences in loneliness scores were found between males and females among the studies. Since this study was a gay, non-Caucasian study, the results of this study are dramatically different from those reported by Russell (1996).

\section{Behavioral Risk Assessment Tool (BRAT)}

The BRAT consists of 61 items scored using categorical and continuous variables. The BRAT was divided into two total scores, sexual risk and alcohol/drug use. Five items pertaining to the number of sexual partners by gender and the years with main partner were excluded from the totaled BRAT sum and alcohol/drug sum. The BRAT sum measured sexual risk behaviors and had 32 items, which were dichotomized into 0 or 1, with total scores ranging from 0 to 18 . Higher scores indicated higher levels of sexual risk behaviors. The mean for the BRAT sum indicating sexual risk was 10.57 ( $S D=$ 3.41). The alcohol/drug sum measured substance use and had 24 items dichotomized into 0 or 1 , with total scores ranging from 0 to 1 . In this study, any score of 1 indicates substance use (drugs, alcohol and/or tobacco). The mean for this study was 0.35 ( $S D=$ .48). Since there are no demarcated scores to indicate unsafe versus safer sexual behaviors, it is necessary to determine the demarcation point between unsafe and safer sex behaviors. The point established as the demarcation point was the mean, $M=10.57$. 
Any total scores less or equal to 11 were interpreted as higher levels of safer sex behaviors, while scores greater than 11 signified lower levels of safer sex behaviors. Several adapted versions of the BRAT have been successfully utilized in research studies (Berkley-Patton et al., 2012; Hawes \& Berkley-Patton, 2014; Gasiorowicz et al., 2005) and all have used different scoring techniques, and not all have reported means. Therefore, the mean for this study cannot be compared to these studies.

\section{Center for Epidemiologic Studies Depression (CES-D) Scale}

The CES-D Scale consists of 20 items rated on a scale of 0 to 3, with total scores ranging from 0 to 60 . In this study, the total CES-D scores ranged from 0 to 43 ( $M=$ $10.70, S D=9.85)$. Scores greater than 16 are indicative of higher levels of depressive symptoms. Since 42 (28\%) of the participants in this study reported total scores above 16, it was noted that they had higher levels of depressive symptoms. Using total scores of 16 and below, 108 (72\%) of the participants in this study reported lower levels of depressive symptoms.

Radloff's (1977) mean from a large sample of Caucasians was noted to be between 7.94 and 9.25. Although the results of this study are different from Radloff's (1977) results, it is relatively similar to those reported by De Santis et al. (2009). De Santis et al. (2009) reported total CES-D scores with HMSM that ranged from 0 to 54 (M

$=15.1, S D=10.7)$, and $55(35.5 \%)$ of those participants had CES-D scores above 16. Additionally, De Santis et al.’s (2009) study participants were mostly foreign-born participants $(N=133,86.9 \%)$, similar to this study $(N=129,86 \%)$. Therefore, this indicates that HMSM may have higher levels of depressive symptoms when compared to 
the general population. Tables 5 to 9 provide a summary of the descriptive statistics of the major study variables.

Table 5.

Descriptive Statistics of the Major Study Variables $(N=150)$

\begin{tabular}{llll}
\hline Instrument & $M$ & $S D$ & Range \\
\hline MSPSS Scale & 4.92 & 1.04 & $1.6-6.5$ \\
UCLA Loneliness Scale & 13.33 & 11.71 & $0-53$ \\
BRAT Sum (Sexual Risk) & 10.57 & 3.41 & $0-18$ \\
BRAT Alcohol/Drug Use & 0.35 & 0.48 & $0-1$ \\
CES-D Scale & 10.70 & 9.85 & $0-43$ \\
\hline
\end{tabular}

Table 6.

Descriptive Statistics of the MSPSS Total Scale Score $(N=150)$

\begin{tabular}{llll}
\hline MSPSS Scale & Range & Frequency & Percent \\
\hline Low Social Support & 1 to 2.9 & 6 & $4 \%$ \\
Moderate Social Support & 3 to 5 & 69 & $46 \%$ \\
High Social Support & 5.1 to 7 & 75 & $50 \%$ \\
\hline
\end{tabular}

Table 7.

Descriptive Statistics of Illicit Drug Use in Past 30 Days $(N=146)$

\begin{tabular}{lll}
\hline Variable & Frequency & Percent \\
\hline No Illicit Drug Use $^{a}$ & 94 & $62.7 \%$ \\
Illicit Drug Use $^{a}$ & 52 & $34.7 \%$ \\
Alcohol $^{b}$ & 106 & $70.7 \%$ \\
Tobacco $^{b}$ & 54 & $36 \%$ \\
\hline
\end{tabular}

Some data are missing: ${ }^{a} n=146 ;{ }^{b} n=147$ 
Table 8.

Descriptive Statistics of the CES-D Scale $(N=150)$

\begin{tabular}{lll}
\hline CES-D Scale Scores & Frequency & Percent \\
\hline Total Scores of 16 and Below & 108 & $72 \%$ \\
Total Scores of Above 16 & 42 & $28 \%$ \\
\hline
\end{tabular}

Table 9.

Descriptive Statistics of HIV Status $(N=150)$

\begin{tabular}{lll}
\hline HIV Status & Frequency & Percent \\
\hline Positive Antibody & 35 & $23.3 \%$ \\
Negative Antibody & 98 & $65.3 \%$ \\
Don't Know Status & 17 & $11.3 \%$ \\
\hline
\end{tabular}

\section{Hypothesis Testing}

\section{Hypothesis One}

The first research question of this study (what effect does social support, loneliness, depression, substance use/abuse, and sexual behaviors have on HIV infection status?) was subjected to hypothesis testing. Hypothesis one stated that social support, loneliness, depressive symptoms, substance use/abuse and sexual behaviors will have a significant effect on HIV infection status. Logistic Regression analysis was used to test hypothesis one .

Logistic Regression is used to test the predictive power of a set of variables and to assess the relative contribution of each individual variable (Pallant, 2010). The dependent variable of interest (HIV infection status) for hypothesis one is categorical; therefore, Logistic Regression is used instead of Multiple Regression because it allows the testing of models to predict categorical outcomes with two or more categories. 
Before hypothesis testing began, correlations between study variables were noted. The two-tailed Pearson product-moment correlation coefficient $(r)$ was used to test directions of relationships and strength among the study variables. Preliminary analyses were performed for all correlations to ensure no violation of the assumptions of normality, linearity, and homoscedasticity.

The relationship between subjective feelings of loneliness (as measured by the UCLA Loneliness Scale) and social support (as measured by the MSPSS Scale) was a moderate, negative correlation between the two variables, $r=-.295, p<.001$, with higher levels of loneliness associated with less social support. The relationship between subjective feelings of loneliness (as measured by the UCLA Loneliness Scale) and depressive symptoms (as measured by the CES-D Scale) was a strong, positive correlation between the two variables, $r=.546, p<.001$, with higher levels of loneliness associated with higher levels of depressive symptoms. The relationship between subjective feelings of loneliness (as measured by the UCLA Loneliness Scale) and sexual risk behaviors (as measured by the BRAT sum) was a small, positive correlation between the two variables, $r=.186, p=.02$, with higher levels of loneliness slightly associated with greater sexual risk behaviors.

The relationship between social support (as measured by the MSPSS Scale) and depressive symptoms (as measured by the CES-D Scale) was not significant, $r=-.128, p$ $=.12$. The relationship between social support (as measured by the MSPSS Scale) and sexual risk behaviors (as measured by the BRAT sum) was not significant, $r=-.086, p=$ .29. These statistics are summarized in Table 10. 
Because the BRAT's alcohol/drug use subscale is a categorical value, an independent-samples t-test was conducted to compare the subjective feelings of loneliness scores (as measured by the UCLA Loneliness Scale) for alcohol/drug users and non-users. There was a significant difference in scores for alcohol/drug users $(M=$ $16.75, S D=11.35)$ and non-users $(M=11.27, S D=11.28) ; t(144)=-2.81, p=.01$, twotailed). The magnitude of the differences in the means (mean difference $=-5.48$, CI: 93.5 to -1.62 ) was very small (eta squared $=.007$ ). A second independent-samples t-test was conducted to compare social support scores (as measured by the MSPSS Scale) and alcohol/drug users and non-users. There was no significant difference in scores for alcohol/drug users $(M=4.88, S D=1.0)$ and non-users $(M=4.97, S D=1.04) ; t(144)=$ 4.83, $p=.63$, two-tailed). A third independent-samples t-test was conducted to compare depressive symptoms scores (as measured by the CES-D Scale) for alcohol/drug users and non-users. There was no significant difference in scores for alcohol/drug users $(M=$ $10.90, S D=9.06)$ and non-users $(M=9.89, S D=9.85) ; t(144)=-.61, p=.54$, twotailed). A forth independent-samples t-test was conducted to compare sexual risk behaviors (as measured by the BRAT sum) for alcohol/drug users and non-users. There was a significant difference in scores for alcohol/drug users $(M=12.23, S D=3.11)$ and non-users $(M=9.74, S D=3.19) ; t(144)=-4.55, p=.001$, two-tailed $)$. The magnitude of the differences in the means (mean difference $=-2.49$, CI: -35.6 to -1.41 ) was very small (eta squared $=.007)$.

Logistic Regression was performed to assess the impact of a number of factors on the likelihood that respondents would report that they are HIV infected. The model contained five independent variables (total scale scores of social support, loneliness, 
depressive symptoms, sexual risk behaviors, and illicit drug use). The full model

containing all predictors was significant, $\chi^{2}(7, N=150)=30.22, p<.001$, indicating that the model was able to distinguish between participants who were HIV-infected and those who were not HIV-infected. Loneliness, social support, depressive symptoms, substance use, and sexual risk behaviors combine to significantly predict HIV infection status, $-2 L L$ $=121.85, p<.001$. The model as a whole explained between 20.6\% (Cox and Snell $\mathrm{R}$ square) and 30\% (Nagelkerke R squared) of the variance in HIV status, and correctly classified $76.3 \%$ of cases. Only two of the independent variables made a unique significant contribution to the model (alcohol/drug use and sexual risk behaviors). The strongest predictor of HIV infection status was alcohol/drug use, recording an odds ratio of 3.21. This indicates that participants who used alcohol/drugs were three times more likely to be HIV-infected than those who did not use alcohol/drugs, controlling for all other factors in the model, $\beta=1.17, p=.017$. An odds ratio of 1.26 for sexual risk behaviors (the BRAT sum) was reported, indicating that participants who had higher sexual risk behaviors were 1.2 times more likely to be HIV-infected, $\beta=0.23, p=.009$.

\section{Hypothesis Two}

The second research question of this study (what effect do selected demographic variables have on social support, depressive symptoms, loneliness, and sexual behaviors?) was subjected to hypothesis testing. Hypothesis two for this study stated that social support, depressive symptoms, and loneliness would have a significant effect on sexual behaviors. Both Logistic Regressions and several two-way, between-groups analysis of variances (two-way ANOVAs) were used to test the second research hypothesis. Partner status and religiosity were the two independent variables 
(categorical) used in the two-way ANOVAs. The two-way ANOVA design allows for testing of the main effect (the joint effect of two independent variables on the dependent variable) and exploration of the possibility of an interaction effect. An interaction effect occurs when the effect of one independent variable on the dependent variable depends on the level of a second independent variable. Since there is variation in the responses provided by the participants regarding religiosity and partner status, using age as a covariance in the models will reduce the unexplained variation. The variable of age is a covariate that is continuous, always observed, and controllable in the study.

Before hypothesis testing began, correlations between study variables were noted for this hypothesis. The two-tailed Pearson product-moment correlation coefficient $(r)$ was used to test directions of relationships and strength among the study variables. Preliminary analyses were performed for all correlations to ensure no violation of the assumptions of normality, linearity, and homoscedasticity. Because some of the variables are categorical, several Chi-square tests for independence $\left(\chi^{2}\right)$ explored those relationships to ensure there was no association between the two variables being measured.

The relationship between participants' age and depressive symptoms (as measured by the CES-D Scale) was a weak, positive correlation between the two variables, $r=.205, p<.05$, with participants who had greater age reporting more depressive symptoms. Chi-square tests for independence (with Yates Continuity Correction) indicated no significant associations between partner status and tobacco use, $\chi^{2}(1, n=147)=2.77, p=.09, p h i=-0.15$, or partner status and illicit drug use $\chi^{2}(1, n=$ 146 ) $=1.21, p=.27, p h i=-0.11$. However, a Chi-square test for independence (with 
Yates Continuity Correction) indicated a significant association between partner status and alcohol use, $\chi^{2}(1, n=147)=7.41, p=.01$, phi $=-0.24$. Three other Chi-square tests for independence (with Yates Continuity Correction) indicated no significant associations between religiosity (religious affiliation) and tobacco use, $\chi^{2}(1, n=147)=0.00, p=1.0$, phi $=-0.17$, or religiosity and alcohol use, $\chi^{2}(1, n=147)=0.36, p=.55$, phi $=0.07$, or religiosity and illicit drug use $\chi^{2}(1, n=146)=0.19, p=.73$, phi $=0.05$.

Three Logistic Regression analyses were performed to assess the impact of chosen demographic variables (partner status and religiosity) on the likelihood that respondents would report alcohol, tobacco, and/or illicit drug use. Each model contained three independent variables (partner status, religion, and age). The first model containing all predictors and alcohol use was significant, $\chi^{2}(3, N=147)=8.49, p=.04$, indicating that the model was able to distinguish between participants who reported and did not report alcohol use. Partner status, religiosity and age combine to significantly predict alcohol use, $-2 L L=165.531, p<.05$. The model as a whole explained between $5.6 \%$ (Cox and Snell R square) and 8.1\% (Nagelkerke R squared) of the variance in alcohol use, and correctly classified $72.1 \%$ of cases. Only one of the independent variables made a unique significant contribution to the model (partner status). The strongest predictor of alcohol use was partner status, recording an odds ratio of 0.4 . This indicates that participants who used alcohol were .5 times more likely to be single, controlling for all other factors in the model, $\beta=-1.04, p=.008$. The second model containing all predictors and tobacco use was not significant, $\chi^{2}(3, N=147)=4.12, p=.25$. Similarly, 
the third model containing all predictors and illicit drug use was not significant, $\chi^{2}$ (3, $N$ $=146)=1.76, p=.62$.

Four, two-way between-groups analysis of variances were conducted to explore the impact of partner status and religiosity on levels of (1) social support, (2) depressive symptoms, (3) loneliness, and (4) sexual behaviors. Age was used as a covariance in the models. The first model analyzed the UCLA Scale and the interaction effect between partner status and religion affiliation group (religiosity) and was not significant, $F$ (1, $145)=.97, p=.33$. The second model analyzed the MSPSS Scale and the interaction effect between partner status and religion affiliation group and was not significant, $F$ (1, $145)=14.15, p=.27$. The third model analyzed the BRAT and the interaction effect between partner status and religion affiliation group and was not significant, $F(1,145)=$ $.03, p=.87$. The fourth model analyzed the CES-D Scale and the interaction effect between partner status and religion affiliation group and was not significant, $F(1,145)=$ .85, $p=.36$. There was a significant main effect for age, $F(1,145)=7.93, p=.006$ and the effect size was small (partial eta squared $=0.05$ ). Therefore, age is a significant predictor of depression after controlling for partner status, and religious affiliation.

\section{Hypothesis Three}

The third research question of this study (what relationship does social support, loneliness, depression and substance use/abuse have with sexual behaviors?) was subjected to hypothesis testing. Hypothesis three stated that social support, loneliness, depressive symptoms and substance use will be significantly related to high risk sexual behaviors. Multiple Regression analysis was used to test this hypothesis. 
Multiple Regression was used to explore the relationship between one continuous variable and a number of independent variables (usually continuous); hence, it will be able to illustrate how well a set of variables is related to a particular outcome. Each independent variable will be evaluated in terms of its relationship over and above other independent variables (Pallant, 2010). Before hypothesis testing began, correlations between study variables were noted. The two-tailed Pearson product-moment correlation coefficients ( $r$ ) were the same as in hypothesis one, and these statistics are summarized in Table 10.

Multiple Regression was performed to assess the impact of a number of factors on the likelihood that respondents would report engaging in high risk sexual behaviors. Preliminary analyses were conducted to ensure no violation of the assumptions of normality, linearity, multicollinearity, and homoscedasticity. Additionally, regression requires a random sample in order to ensure that the conclusions generated by the sample are representative of the reality in the population. Since this study incorporated a convenience sample, caution should be used when interpreting theses results, particularly when relating the results of this study to the general population of middle-aged HMSM. While violating the assumption of random sampling will not impact parameter estimates, it may affect standard errors and thus yield inaccurate statistical results (Darlington, 1990).

The model contained five independent variables (total scale scores of social support, loneliness, depressive symptoms, sexual risk behaviors, and alcohol/drug use) and was significant, $R^{2}=.211, F(6,139)=6.19, p<.001$, indicating that depression, substance use, social support, and loneliness combine to explain $21.10 \%$ of the variance 
in sexual risk behaviors. Significant relationships include alcohol use $(\beta=1.38, p=$ $.030)$ and illicit drug use $(\beta=1.95, p=.001)$. Loneliness $(\beta=0.54, p=.063)$, tobacco use ( $\beta=0.52, p=.927)$, social support $(\beta=-0.25, p=.349)$, and depressive symptoms ( $\beta$ $=-0.06, p=.075$ ) were not found to be significantly related to sexual behaviors.

Table 10.

Bivariate Correlations Between Major Study Variables $(N=150)$

\begin{tabular}{|c|c|c|c|}
\hline & $\begin{array}{c}\text { UCLA } \\
\text { Loneliness Scale }\end{array}$ & BRAT Sum & CES-D \\
\hline $\begin{array}{l}\text { UCLA } \\
\text { Loneliness } \\
\text { Scale }\end{array}$ & & $.186^{*}$ & $.546^{* *}$ \\
\hline $\begin{array}{l}\text { MSPSS } \\
\text { Scale }\end{array}$ & $-.295^{* *}$ & -.086 & -.128 \\
\hline
\end{tabular}

\section{Summary of Chapter 4}

A convenience sample of 150 urban HMSM aged 40 to 65 provided the sample for this study. Over two-thirds $(86 \% ; n=129)$ were foreign-born, with 18.09 years $(S D=$ $15.97)$ as the mean length of time residing in the US. More than half $(62.7 \% ; n=94)$ were single and not involved in any relationship. The sample consisted of a large number of men who have at least a high school education $(51.3 \% ; n=77)$.

Three hypotheses in this study were tested with statistical analyses. Hypothesis one was supported, as loneliness, social support, depressive symptoms, substance use, and sexual risk behaviors had an influence on HIV infection status when subjected to Logistic Regression. Hypothesis two was only partially supported, as partner status, religiosity and age combined to have an influence on alcohol use in the study's 
participants when subjected to Logistic Regression. Additionally, it was found that age is a significantly related to depression, i.e., increased age among participants was associated with increased depressive symptoms. Hypothesis three was supported, as depressive symptoms, substance use, social support, and loneliness did have an influence on sexual risk behaviors when subjected to Multiple Regression. Significant relationships included alcohol use and illicit drug use.

The statistical analyses of the study's data allowed this researcher to draw some conclusions about the study's variables. First, loneliness, social support, depressive symptoms, alcohol/drug use, and sexual risk behaviors do have an influence on HIV infection status in this study. Second, partner status, religious affiliation and age do have an influence on alcohol use in this study. Also, participants with increased age have increased depressive symptoms in this study. Lastly, depressive symptoms, substance use, social support, and loneliness do have an influence on sexual risk behaviors in this study, specifically alcohol use and illicit drug use. 


\section{CHAPTER 5-DISCUSSION AND CONCLUSION \\ Introduction}

This chapter summarizes the study, and presents the findings in relationship to the study variables of social support, loneliness, depressive symptoms, substance use, and sexual behaviors. This chapter will discuss predictors of sexual behaviors for this sample of middle aged HMSM. Limitations of the study will be discussed. Implications for nursing education, research, policy, and practice are also included in this chapter.

\section{Summary of the Study}

MSM and bisexual men are more severely affected by HIV infection than any other group in the US, with HMSM bearing a disproportionate burden of HIV infection (CDC, 2012). Sexual risk behaviors, such as number of lifetime sex partners, rate of partner exchange, and frequency of unprotected sex, may contribute to rates of STIs and HIV infection among HMSM (CDC, 2015). HMSM are disproportionately affected by HIV infection and only a few behavioral interventions address prevention needs, which have historically resulted in a minimal effect, or no effect with reducing STIs and HIV infection among HMSM (Adam, Betancourt, \& Serrano-Sanchez, 2011; CaraballoDieguez et al., 2005; O’Donnell et al., 2014; Sikkema et al, 2014; Toro-Alfonso, VarasDíaz, \& Andújar-Bello, 2002; Vega, Spieldenner, DeLeon, Nieto, \& Stroman, 2011). Research with middle age HMSM may provide some insight as to what characteristics, e.g., social support, loneliness, depressive symptoms, substance use, demographic variables or characteristics, might influence sexual behaviors. The significant findings from this research could then be used to develop tailored risk reduction interventions that may help decrease STI and HIV infection rates in this population. 
A review of the literature found some studies that documented the characteristics and experiences that affect subsequent high risk sexual behaviors, such as engaging in UAI, drug and alcohol use for HMSM (De Santis et al., 2011; De Santis et al., 2014; De Santis et al., 2015; De Santis et al., 2012; Fernandez et al., 2009; Fernandez et al., 2007). However, the data regarding the relationship of social support, loneliness and depressive symptoms on sexual behaviors for HMSM was unclear. In addition, no research was found exclusively on middle age HMSM and the limited research found on the effects of social support, loneliness and depressive symptoms on sexual behaviors was with smaller samples of HMSM (De Santis et al., 2012; De Santis et al., 2009; De Santis et al., 2011;

De Santis et al., 2014; Fernandez et al., 2007; Fernandez et al., 2009; Guarnero, 2007; Lauby et al., 2012; Rhodes et al., 2011; Vasquez et al., 2011). Many of the studies revealed several co-occurring disorders that precipitate engagement in high risk sexual behaviors (Carballo-Dieguez et al., 2005; De Santis et al., 2012; De Santis et al., 2015; Guarnero, 2007; Lo et al., 2011; O’Donnell et al., 2014).

\section{Theoretical Framework}

The theoretical framework, which guided this study is Singer's Syndemic Theory (1996). This model contends that co-occurring health conditions interact synergistically, producing adverse health outcomes and inequalities, including susceptibility to disease. Certain psychosocial issues, i.e., social support, loneliness, depressive symptoms, and substance use, are additive effects that create a greater increase in sexual risk-taking behaviors, which may lead to STI and HIV infection vulnerability. In addition, addressing one aspect of the syndemic alone is not enough to change or modify health behavior, but intertwined circumstances and the social environment, e.g., demographic 
variables (partner status, religious affiliation, HIV infection status), in which they occur must be equally considered.

\section{Hypotheses}

Developed from the theoretical framework guiding this study, the following three research hypotheses were generated and tested in this study:

Hypothesis 1: Middle age HMSM (45-65 years of age) with decreased social support and increased loneliness may be at risk for depression and substance use/abuse leading to high risk sexual behaviors making them susceptible to HIV infection.

Hypothesis 2: Middle age HMSM with social connections and support are less depressed, lonely, and practice safer sex behaviors.

Hypothesis 3: Middle age HMSM with social connections and support and less psychosocial problems, such as substance use/abuse, depression, and loneliness, report lower levels of high risk sexual behaviors, such as UAI and drug/alcohol use during sexual activity.

To determine the relationships between the study variables and demographic variables, this study employed a non-experimental, cross-sectional research design with a convenience sample of urban middle aged HMSM. Data were collected with anonymous, self-administered questionnaires from urban middle aged HMSM $(N=150)$ at various sites in South Florida over a 5-month period in 2015. The Statistical Package for the Social Sciences (SPSS) 22.0 was used for data analyses. The hypotheses were subjected to testing using the Pearson product-moment correlation coefficients $(r)$, chi-square test

for independence $\left(\chi^{2}\right)$, independent-samples $t$-test, Logistic Regressions, two-way, 
between-groups analysis of variances (two-way ANOVAs), and Multiple Regression analyses.

The sample for this study included 150 middle aged HMSM with ages ranging from 40 to 60 years $(M=45.54, S D=4.71)$. The sample consisted of a large number of foreign-born men ( $86 \% ; n=129)$, with 18.09 years $(S D=15.97)$ as the mean length of time residing in the US. In addition, more than half $(62.7 \% ; n=94)$ of the sample was single and not involved in any relationship. Data from this study support Hypothesis One (loneliness, social support, depressive symptoms, substance use, and sexual risk behaviors did have a statistical influence on HIV infection status); partially support Hypothesis Two (partner status, religiosity and age combined to have a statistical influence on alcohol use); and support Hypothesis Three (depressive symptoms, substance use, social support, and loneliness did have a statistical influence on sexual risk behaviors).

\section{Discussion of Findings}

\section{Demographic and Background Characteristics}

The study's main data collection setting was Miami-Dade County, Florida. Because a convenience sampling design was used in this study, it is necessary to compare the study’s demographic findings with the main data collection setting, i.e., Miami-Dade County. An interesting demographic variable in this study is the Hispanic subgroups representing the collected sample. According to the US Census Bureau's (2011) report, 63\% of Miami’s residents communicate in Spanish at home, and 51.3\% are foreign-born whereas $19.4 \%$ of the total population of Hispanics in the US is foreign-born. In 2011, the US Census Bureau reported that the largest Hispanic origin of individuals living in 
the US were Mexican (63\%), Puerto Rican (9.2\%), Cuban (3.5\%), and Dominican (2.8\%), as compared to 34.3\% Cuban, 4.6\% Columbian, 4.2\% Nicaraguan, 3.7\% Puerto Rican, 2.3\% Dominican, and 2.1\% Mexican in Miami-Dade County.

One of the disadvantages of using a convenience sampling design is that truly representative samples of the population may not occur (Salkind, 2013). The results for the following demographics were not surprisingly because of the demographics of Miami-Dade County. The participants in this study were $86 \%(n=129)$ foreign-born, of which $48.7 \%(n=73)$ had at least an associate's degree. The Hispanic subgroups in this study were composed of the following: $34.7 \%(n=52)$ Cubans; $14 \%(n=21)$ Americanborn; 10.7\% ( $n=16)$ Columbians; 6.7\% $(n=10)$ Venezuelan; 6\% $(n=9)$ Argentine; 5.3\% ( $n=8)$ Peruvian; 4.7\% $(n=7)$ Puerto Rican; 4\% $(n=6)$ Dominican; 3.3\% $(n=5)$ Guatemalan; 2\% $(n=3)$ Mexican. The Hispanic subgroups of Chilean, Panamanian, Nicaraguan, and Salvadorian each had 1.3\% $(n=2)$ and the subgroups of Ecuadorean, Honduranean, Paraguayan, and Uruguayan each had 0.7\% $(n=1)$.

The instruments in the study were available in Spanish and English. Only 46\% ( $n$ = 69) of the participants chose to complete the study in Spanish. Even though 86\% ( $n=$ 129) of the sample was foreign-born, language preference was not predominantly Spanish. This may give some indication of the assimilation status of the participants. Although this is not a measure of acculturation, these results seem to indicate that approximately half of the sample is not fully assimilated to US culture, while the other half may be assimilated. In addition, the participant's length of time in the US was reported as $M=18.09(S D=15.97)$. 
The US Census Bureau does not ask direct questions about sexual orientation; however, the last US Census Bureau survey included the ability to self-disclose same-sex partners/spouses residing in same households. The number of gay and lesbian households reported in South Florida has increased sharply over the past 10 years, and the number of same-sex partner households has increased 60\% in Florida from 2000 to 2010. In 2010, 7, 436 same-sex partner households were reported for Miami-Dade County (US Census Bureau, 2011). This study’s participants were recruited in MiamiDade County exclusively. The relationship status of the participants in this study noted that 37.3\% ( $n=56)$ were involved in an intimate relationship. However, this statistic does not have a comparison since the US Census Bureau does not report actual County percentages for same sex couples. However, De Santis et al.'s (2012) descriptive crosssectional study of 135 HMSM from South Florida with a mean age of $37.53(S D=8.12)$ reported that $60.7 \%(n=82)$ were involved in a primary relationship.

This study's recruitment of participants was conducted at various community sites in Miami-Dade County. The majority of participants were recruited from bars/clubs ( $n=$ 49; 32.7\%), followed by South Florida’s Hispanic LGBT Pride Festival-Orgullo ${ }^{\circledR}(n=$ 39; 26\%), flyer responses ( $n=19 ; 12.7 \%)$, restaurants ( $n=18 ; 12 \%)$, snowball sampling ( $n=17 ; 11.3 \%$ ), and hair salons $(n=8 ; 5.3 \%)$. Therefore, $72 \%$ of participants were predominantly recruited in social settings (bars/clubs and festivals).

\section{Relationship Between Major Study Variables}

Results of the statistical analysis conducted to test Hypothesis One (social support, loneliness, depression, substance use/abuse, and sexual behaviors will have a significant effect on HIV infection status) in this study indicated that there was a 
significant relationship between loneliness, social support, depressive symptoms, substance use, sexual risk behaviors, and HIV infection status. In terms of supportive social relationships and HIV infection, the study's findings were consistent with Lauby et al.’s (2012) study which reported that MSM who scored higher on the supportive relationship index had significantly lower odds of testing HIV antibody positive in the study, $A O R=0.68$. In terms of loneliness, the study's findings were consistent with Rhodes et al.’s (2011) qualitative study, which reported high levels of loneliness and social isolation were related to HIV infection risk for HMSM. However, De Santis et al.'s (2014) qualitative study did not exclusively find themes of loneliness linked to HIV infection, which is inconsistent with this study's results. However, De Santis et al. (2012) did report that a lack of psychosocial support for HMSM resulted in high risk sex, substance abuse, and violence, consequentially increasing risk for HIV infection.

Consistent with this study finding, De Santis et al. (2012) reported that higher levels of depression were reported with higher levels of drunkenness and alcohol use as opposed to lower levels of depression $(t=-2.41, p=0.023)$, and higher levels of depression were reported with drug use in past three months $(t=-1.75, p=0.023)$. Additionally, De Santis et al. (2012) reported higher levels of depression were associated with being intoxicated (from drug or alcohol) before sexual activity in past 3 months ( $t=$ $-3.25, p=0.003)$. However, in a study conducted by Vasquez et al. (2011) the only variables that significantly predicted substance abuse were age ( Wald $\chi 2=12.13, p<$ 0.001 ) and depression ( Wald $\chi 2=6.16, p=0.00$ ), with older participants being less likely to report substance abuse in the past three months $(A O R=0.93,95 \% C I=0.89,0.97)$, 
and participants with increased depressive symptoms being more likely to report substance abuse $(A O R=1.05,95 \% C I=1.01,1.09)$.

The results of the statistical analysis conducted to test Hypothesis Two (demographic variables, partner status and religiosity, will have an effect on social support, depressive symptoms, loneliness, and sexual behaviors) in this study indicated that certain demographic variables have a significant effect on substance use and depressive symptoms, but no significant relationships were noted with social support loneliness, or sexual behaviors. Differences existed in terms of partner status, religiosity and age on alcohol use in the study's participants. Additionally, it was found that age was a significant predictor of depression, i.e., increased age among participants resulted in increased depressive symptoms. Results indicated that it is possible to predict substance use and depressive symptoms, but not social support, loneliness or sexual behaviors by certain demographic variables.

The results of the statistical analysis conducted to test Hypothesis Three (social support, loneliness, depression and substance use will have an effect on sexual behaviors) in this study indicated that there was a significant relationship between depressive symptoms, substance use, social support, loneliness and sexual risk behaviors. Results support De Santis et al.'s (2012) South Florida study, which reported mental health, expressed in terms of depression and self-esteem, were strong predictors of sexual behaviors among HMSM, of which alcohol abuse (18\%), depression (25\%) and other variables not measured in this study acted as the major contribution of predictors of sexual behaviors. Similarly, in a study of South Florida HMSM, Fernandez et al. (2009) reported that substance-using HMSM had more sexual partners $(n=7)$ and more UAI 
(58\%) in the last 6 months than non-substance-using HMSM (sex partners $n=3 ; 36 \%$

UAI). Substance users reported higher levels of loneliness $(2.37, S D=0.92)$, more psychological distress $(2.49, S D=1.22)$, more experiences of racism $(1.33, S D=0.36)$, and less social connections $(2.41, S D=0.48)$ than did non-drug users, who had the following scores: loneliness $2.14, S D=0.82$; psychological distress $2.18, S D=1.14$; racism 1.24, $S D=0.30$; and social connections $2.38, S D=0.44$ (Fernandez et al., 2009).

\section{Limitations of the Study}

The results from this study of urban middle age HMSM provide some insight into the relationships of variables that could be used to predict sexual behaviors of the study's participants. Results from the study may be the first step in developing interventions targeted to reduce STI and HIV infection rates among middle aged HMSM. These results can contribute to the knowledge base of what is known about this population and can direct programs of health education geared to decrease high risk sexual practices, which will subsequently reduce morbidity and mortality of this high risk group. However, this study is not without limitations. In addition to the limitations described in Chapter One, the following are identified limitations:

1. The non-experimental, cross-sectional research design of the study limits generalizability to the general population (Salkind, 2013). The study's results cannot be generalized to the larger population of middle age HMSM.

2. A convenience sampling design was used in this study. One of the disadvantages of using a convenience sampling design is that truly representative samples of the population may not occur (Salkind, 2013). 
Additionally, $11.3 \%$ ( $n=17)$ of the participants were recruited by snowball sampling, which can hinder the generalizability of the findings to the HMSM population.

3. The time period of data collection was from July 2015 to December 2015. There may have been a different pool of participants if data were collected at a different time frame or for a longer period of time.

4. This study included self-administered sexual and mental health surveys, which can have an inherent risk of reporting bias and disclosure of sexual risk behaviors. In addition, participants were told that the surveys were checked for completeness prior to awarding the stipend, this may have created socially desirable responses.

5. Many community venues catering to or frequented by HMSM were not used in this study. These included immigration offices, local stores, places of worship, and community agencies. Additionally, the study's sample was only collected in Miami-Dade County. Immigration offices, local stores, and places of worship were not used because this researcher could not establish permission from these venues to access this population. Community agencies and other counties in South Florida were not used because the established sample size was reached before using these sites. Eliminating these sites for data collection may have reduced the diversity of the study's sample. Because of the unique characteristics of Miami-Dade County, there is a possibility that findings from this study may not be fully generalizable to other metropolitan areas. 
6. During the data collection for this study, this researcher was present at all times. Participants may have been influenced by the presence of the researcher and may have responded to the self-administered questionnaire in a manner perceived to be more socially acceptable.

7. Participants received a small monetary contribution for completing the questionnaire, which may have influenced the results. Participants may have felt obligated to complete the entire survey to receive the stipend.

\section{Implications for Nursing}

As a population, HMSM are disproportionately affected by HIV infection and are at risk for increased mortality and morbidity due to socioeconomic factors, such as lack of access to healthcare, lower educational accomplishments, language barriers, and increased poverty (CDC, 2011; CDC 2013). Research with HMSM has identified a number of factors, such as depression, substance use, and acculturation, which influence high risk sexual behaviors (De Santis et al., 2012; De Santis et al., 2009; De Santis et al., 2011; De Santis et al., 2014; Fernandez et al., 2007; Fernandez et al., 2009; Guarnero, 2007; Lauby et al., 2012; Rhodes et al., 2011; Vasquez et al., 2011). UAI is a preventable high risk sexual behavior that increases STI and HIV infection rates in HMSM (CDC, 2011). Tailored risk reduction interventions and strategies can be developed to reach this population and potentially reduce the consequential effects of high risk sexual behaviors, thereby decreasing STI and HIV infection transmission.

Because $86 \%(n=129)$ of this study's sample are HMSM who were foreign-born, it is unclear if they have received knowledge on STI or HIV infection transmission, risk factors, and long-term consequences of HIV infection. Participants' country of origin 
may not have provided this education, so it is unclear if the participants perceive themselves at risk for STI or HIV infection by engaging in certain sexual behaviors. Additionally, this researcher cannot assume that participants have received knowledge or education on STI and HIV infection even though the mean length of time in the US for this sample is 18.09 years $(S D=15.97)$.

\section{Education}

This study’s findings have implications for nursing education. As the population of Hispanics increase, so do the sexual minorities within that subgroup; therefore, it is crucial for nurses to be aware of not only ethnic diversities but the consequential risk factors and health disparities associated with sexual minorities. When caring for the middle aged HMSM population, one of the most important roles of the nurse is that of an educator. Content on HIV infection and STIs is currently included in all nursing curricula. This ensures that all graduating nurses have had instruction on the care of individuals affected by HIV/STIs, as well as those at risk for HIV/STIs. This content may need to be reinforced for practicing nurses as care and treatment for those with HIV infection continue to evolve. Because nurses have the responsibility of educating all clients about HIV infection risk as part of health promotion and disease prevention, all clients should be encouraged to discuss sexual risk factors with nurses, and to engage in HIV counseling and testing as recommended (CDC, 2013).

Since nurses are the main direct caregivers in many healthcare settings, nursing students, practicing nurses and advanced practice nurses are in a pivotal position to improve healthcare for LGBT patients. Gaps in nursing curricula should be modified to address knowledge dissemination related to LGBT issues and the health disparities 
affecting this population. Nurses should aware of the potential issues related to sexual orientation, so that effective healthcare can be delivered to all populations. Nursing schools should incorporate curricula that reflect changing social norms with regard to the growing acceptance of gender diversity and sexual minorities (Lim, Brown, \& Jones, 2013).

Marginalized populations, such as middle aged HMSM, are disproportionally affected by HIV infection, yet are the least likely to have access to HIV prevention, testing, and treatment services because of widespread stigma and discrimination (CDC, 2013). Hughes (2009) found that a majority of lesbians and gays were concerned that their sexuality or gender identity may affect the quality of health services received. Since HIV infection remains a highly stigmatized condition and continues to be blamed on irresponsible personal behavior, HIV-infected individuals or those at risk for HIV infection may have feelings of stigma and marginalization. Therefore, nurses should be culturally sensitive and educated on the vulnerability, stigma, and psychological stress of Hispanic sexual minorities.

\section{Practice}

Nursing has depended on formal knowledge and research as a basis for practice. Nursing practice often embraces a holistic approach, which includes biological, psychological and spiritual aspects of health and illness. Additionally, nurses promote healthy behaviors as part of disease prevention efforts (American Nurses Association [ANA], 2010). Therefore, this study’s findings can help nurses develop, implement, and evaluate STI and HIV infection prevention programs that can target middle aged HMSM. 
Most people are not raised to discuss sexuality and sexual matters openly. Individuals may have difficulty talking to healthcare providers about sexual health and nurses may have difficulty promoting sexual health in healthcare environments (De Santis \& Vasquez, 2010). Nurses often lack comfort and confidence in addressing human sexuality issues and often perceive that patients do not expect nurses to address these concerns (De Santis \& Vasquez, 2010). Nurses are often uncomfortable because they lack the education and skills to address sexuality issues. Research has shown that a lack of patient/healthcare provider communication about human sexuality issues is a risk factor for STIs and HIV infection (Morris, 2012). Incorporating education and skills in regards to listening, interpreting and clearly replying to patients' sexual health concerns can promote trusting relationships, candid communication, and facilitate discussion on sexuality and sexual health in nursing practice.

Another difficulty that nurses may encounter in the practice setting is screening for substance use and mental health issues. It is important for nurses to identify people at risk and approach interventions and treatment options with these patients. It is important to distinguish from physical and psychological dependence issues, since patients may have co-occurring mental health problems. According to Andrews (2008), interactions between nurses and patients may be influenced by the degree of intimacy desired, which may range from very formal interactions to close personal relationships. Nurses should be sensitive to the potentially stigmatizing nature of screening for HIV/STIs, substance use, and mental health issues. Developing and possessing interviewing skills that establish rapport with individuals while being sensitive may help gather information 
about individuals' lifestyle and subsequent health behaviors that place individuals at risk for STI and HIV infection.

It is essential that middle aged HMSM have an accurate perception of risk for HIV infection. HIV risk perception may influence sexual behaviors. Knowledge of HIV and an accurate perception of HIV risk may influence HIV testing. In addition to behaviors, knowledge, and perceptions, the relationship of sexual behaviors and mental health should be addressed with middle aged HMSM. Therapy sessions addressing high risk sexual behaviors with a culturally appropriate context for middle aged HMSM may discover factors such as depression, loneliness, and substance use which may interfere with intentions to practice safer sex.

The results of this study have cultural implications for nursing practice. Potocky, Dodge, and Greene (2007) reported that a lack of provider cultural competence can compound barriers to healthcare by resulting in differential treatment of immigrants by healthcare providers and health care delivery systems. Limited research has been conducted with middle aged HMSM, specifically foreign-born men. A myriad of factors exists within the Hispanic culture in regards to education, religiosity, beliefs, values, experiences, and political views, which can ultimately affect middle aged HMSM risk behaviors. These men face unique challenges that may impact their physical and mental health (De Santis et al., 2009). Very little is known about their social support networks and self-efficacy intentions to participate in safer sexual behaviors. This study fills some gaps and provides some insight of social support, feelings of loneliness, depressive symptoms, substance use and sexual behaviors in the knowledge base for middle aged HMSM. 


\section{Policy}

In the US, the population of middle aged HMSM HIV infected individuals continues to grow. High incidences of comorbidities due to antiretroviral therapy (ART), age-related and other diseases introduce new challenges for policy makers. In 2015, the CDC recommended routine HIV infection testing be offered to those ages 13 to 64 as a standard of general health care, and included in the general consent for care and treatment.

Policy barriers to HIV infection screening must be adequately and appropriately addressed with concentrated, sensitive, and respectful efforts (DeMarco, Gallagher, Bradley-Springer, Gracia Jones, \& Visk, 2012). On a federal and state level, nurses should advocate for policies supporting HIV infection testing and it should be the standard rather than the exception. Regardless of age, gender, race/ethnicity, sexual orientation, or socio-economic status, HIV infected individuals should have access to high quality, life-preserving care free from stigma and discrimination (DeMarco et al., 2012).

As stated earlier, the 2011 US census report notes that the 65 year and older population is growing faster than younger groups, projects the Hispanic population to double by 2050, and estimates the LGBT community to be 9 million. However, being middle age has been long associated with a personalization of mortality. Aging has also been associated with anxieties about sexual inadequacies and performance capabilities in men, especially in societies that promote sexually youth oriented lifestyles (Kertzner, 2014). 
In 2005, Price found that older gay men and lesbians were found to be excluded from policy, practice and research perspectives; additionally, this invisibility showed that little is known regarding the needs of older gay men and lesbians. In today's world, modern medical advancements such as pre-exposure prophylaxis (PrEP) reduce HIV infection transmission. PrEP is an HIV infection prevention strategy in which HIV noninfected individuals take antiretroviral medication prophylactically prior to sexual exposure to reduce risk for HIV infection (CDC, 2014). However, the majority of media campaigns for PrEP target younger gay men (CDC, 2014). Advocating for PrEP with older HMSM populations can have a profound impact on their health by increasing the likelihood of healthy aging among this population and consequently decrease their health disparity.

\section{Research}

Despite repeated efforts focusing the effects of substance use during sex and advocating for condom use, it has not been enough to decrease STI and HIV infection rates among middle aged HMSM. Research contributing to the roots of high risk sexual behaviors, such as UAI and substance use during sexual encounters, can close gaps between theory and practice while reducing this incidence. Conducting research with other mental health issues besides depression and loneliness can gain insight to develop interventions geared to high risk sexual behaviors in the HMSM population.

Previous research with the general population of HMSM has identified factors such as acculturation, depression, low self-esteem, and other mental health conditions that are related and may contribute to substance use (Gonzalez-Guarda et al., 2011; Gonzalez-Guarda et al., 2010). Research with middle aged HMSM should also include 
examining other forms of support outside the family, i.e., gay community involvement and gay support networks, including friends. Most of these support network members know about the individuals' sexual orientation, thereby possibly establishing emotionally fulfilling and meaningful intimate connections with other MSM. These social connections may also help explore venues for sexual connections that are safe and satisfying and elevate feelings of self-worth, while reducing marginalization.

In addition to mental health issues and substance use, research suggests impulsivity (a spur of the moment act without regard to future consequences), and compulsivity (sexual fantasies and behaviors increasing in intensity and frequency over time which interfere with personal and interpersonal pursuits) are associated with high risk sexual behaviors and substance use among MSM (Parsons, Grov, \& Golub, 2012; Semple, Zians, Grant, \& Patterson, 2006). De Santis et al. (2012) reported that HMSM need to have experienced numerous sexual partners in their sexual history to feel superior and experience self-worth. Parsons et al. (2012) found sexual compulsivity as a syndemic factor related to HIV infection acquisition and high risk sexual behavior among urban MSM; furthermore, sexual compulsivity was associated with being HIV antibody positive and reporting greater incidence of UAI. Future research with middle aged HMSM should include impulsivity and compulsivity, which are impairing traits that have an additive effect on high risk sexual behaviors.

As stated previously, HMSM are disproportionately affected by HIV infection and only a few behavioral interventions have addressed their prevention needs. Interventions developed to decrease STIs and HIV infection in middle aged HMSM have been shown to produce minimal effect, or no effect (Adam et al., 2011; Caraballo- 
Dieguez et al., 2005; O’Donnell et al., 2014; Sikkema et al., 2014; Toro-Alfonso et al., 2002; Vega et al., 2011). Since Hispanics tend to have sex with partners of the same race/ethnicity, Hispanics face a greater risk of HIV infection (Bianchi, Brooks, \& Reisen, 2010; CDC, 2013). Future research on developing effective behavioral interventions focusing on sexual safety, condom use and partner negotiation, for use with culturally diverse middle aged HMSM may reduce high risk sexual behaviors and promote HIV antibody testing.

\section{Summary of Chapter 5}

The purpose of this study was to determine the relationship of social support, loneliness, depressive symptoms, substance use, and high risk sexual behaviors in middle aged HMSM. Some insight into psychological and sexual behaviors that are specific to middle aged HMSM was obtained from this research study. Singer’s Syndemics Theory (1996) provided this study's theoretical framework to research middle aged HMSM and the study’s major concepts. The findings from this study should be used to assess, diagnose, plan, implement and evaluate prevention strategies geared to reduce STI and HIV infection in this population. Future research should build on these findings and develop tailored risk reduction interventions addressing HMSM, with particular attention to the understudied age group of the middle aged HMSM. 


\section{REFERENCES}

Adam, B. D., Betancourt, G., \& Serrano-Sanchez. (2011). Development of an HIV prevention and life skills program for Spanish-speaking gay and bisexual newcomers to Canada. The Canadian Journal of Human Sexuality, 20(1-2), 1117.

Adekeye, O. A., Heiman, H. J., Onyeabor, O. S., \& Hyacinth, H. I. (2012). The new invincibles: HIV screening among older adults in the US. PLOS ONE, 7(8): e43618. doi:10.1371/journal.pone.0043618

American Academy of Pediatrics. (2010). Multidimensional scale of perceived social support (Spanish). Addressing Mental Health Concerns in Primary Care: A Clinician's Toolkit. Retrieved from http://www.heardalliance.org/wpcontent/uploads/2011/04/Multidimensional-Scale-of-Perceived-Social-SupportSpanish.pdf

American Nurses Association (ANA). (2010). Nursing's social policy statement: The essence of the profession. Retrieved from nursingworld.org/social-policystatement

Andrews, M. M. (2008). Culturally competent nursing care. In M. Andrews \& J. Boyle (Eds.), Transcultural concepts in nursing care (pp. 15-33). Philadelphia: Lippincott Williams \& Wilkins.

Arseniou, A., Arkaterini, A., \& Samakouri, M. (2013). HIV infection and depression. Psychiatry and Clinical Neurosciences, 68(2), 96-109. doi:10.1111/pcn.12097

Bachman, J. G., O'Malley, P. M., \& Johnston, L. D. (1979). Developing composite measures of drug use: Comparisons among lifetime, annual, and monthly prevalence reports for thirteen classes of drugs (Monitoring the Future Occasional Paper No. 5.) Ann Arbor, MI: Institute for Social Research.

Balan, I. C., Carballo-Dieguez, A., Ventuneac, A., Rermien, R. H., Dolezal, C., \& Ford, J. (2013). Are HIV-negative men who have sex with men and who bareback concerned about HIV infection? Implications for HIV risk reduction interventions. Archives of Sexual Behavior, 42(2), 279-289. doi:10.1007/s10508011-9886-2

Beck, A., Steer, R., \& Brown, G. (1996). The Beck Depressive Inventory manual (2 ${ }^{\text {nd }}$ Ed.). San Antonio, TX: The Psychological Corporation. 
Berkley-Patton, J., Moore, E. W., Hawes, S. M., Thompson, C. B., \& Bohn, A. (2012). Factors related to HIV testing among an African American church-affiliate population. AIDS Education and Prevention, 24(2), 148-162. doi:10.1521/aeap.2012.24.2.148

Bianchi, F. T., Brooks, K. D., \& Reisen, C. A. (2010). Partner selection among Latino immigrant men who have sex with men. Archives of Sexual Behaviors, 39, 13211330. doi:10.1007/s10508-009-9510-X

Blashill, A. J., \& Vander Wal, J. S. (2010). Gender role conflict as a mediator between social sensitivity and depression in a sample of gay men. International Journal of Men's Health, 9(1), 26-39. doi:10.3149/jmh.0901.26

Borges, A., Prieto, P., Ricchetti, G., Hernandez-Jorge, C., \& Rodriguez-Naveiras, E. (2008). Validación cruzada de la factorización del test UCLA de soledad. Psicothema, 20(4), 924-927.

Burns, N., \& Grove, S. K. (2009). The Practice of Nursing Research: Appraisal, Synthesis, and Generation of Evidence. (6 ${ }^{\text {th }}$ ed.). Philadelphia, PA: Saunders.

Carballo-Dieguez, A., Dolezal, C., Leu, C. S., Nieves, L., Diaz, F., Descena, C., \& Balan, I. (2005). A randomized controlled trial to test an HIV-prevention intervention for Latino gay and bisexual men: Lessons learned. AIDS Care, 17(3), 314-328. doi: 10.1080/09540120512331314303

Centers for Disease Control and Prevention (CDC). (2015, November). 2014 sexually transmitted diseases surveillance: STDs in men who have sex with men. Retrieved from http://www.cdc.gov/std/stats14/msm.htm

Center for Disease Control and Prevention (CDC). (2011, October). CDC fact sheet: HIV and AIDS among Latinos. Retrieved from http://www.cdc.gov/hiv/latinos/index.htm

Center for Disease Control and Prevention (CDC). (2012a). HIV in the United States: At a glance. Retrieved from http://www.cdc.gov/hiv/resources/factsheets/us.htm

Center for Disease Control and Prevention (CDC). (2013, November). HIV in the United States: At a glance. Retrieved from http://www.cdc.gov/hiv/statistics/basics/ataglance.html

Center for Disease Control and Prevention (CDC). (2015, September). HIV testing in clinical settings. Retrieved from http://www.cdc.gov/hiv/testing/clinical/

Centers for Disease Control and Prevention (CDC). (2012). Minority health. Retrieved from http://www.cdc.gov/minorityhealth/populations/atrisk.html 
Center for Disease Control and Prevention (CDC). (2014). Preexposure prophylaxis for the prevention of HIV infection in the United States-2014: A Clinical practice guideline. http://www.cdc.gov/hiv/pdf/guidelines/PrEPguidelines2014.pdf

Centers for Disease Control and Prevention (CDC). (2013). Recommended prevention services. Retrieved http://www.cdc.gov/hiv/prevention/programs/pwp/risk.html

Chen, Z., Branson, B., Ballenger, A. M., \& Peterman, T. A. (1998). Risk assessment to improve targeting of HIV counseling and testing services for STD clinic patients. Sexually Transmitted Diseases, 25(10), 539-543.

City-Data. (2012). Miami-Dade county data. Retrieved on October 13, 2015 from www.city-data.com/forum/miami/

Cohen, J. (1987). Statistical Power Analysis for the Behavioral Sciences. Hillsdale, NJ: Lawrence Erlbaum Association.

Cohen, S. (1988). Psychosocial models of the role of social support in the etiology of physical disease. Health Psychology, 7(3), 269-297.

Darlington, R. B. (1990). Regression and linear models. New York, NY: McGraw Hill.

DeLonga, K., Torres, H. L., Kamen, C., Evans, S. N., Lee, S., Koopman, C., \& GoreFelton, C. (2011). Loneliness, internalize homophobia, and compulsive Internet use: Factors associated with sexual risk behavior among a sample of adolescent males seeking services at a community LGBT center. Sexual Addiction \& Compulsivity, 18, 61-74. doi:10.1080/10720162.2011.581897

DeMarco, R. F., Gallagher, D., Bradley-Springer, L., Gracia Jones, S., \& Visk, J. (2012). Recommendations and reality: Perceived patient, provider, and policy barriers to implementing routine HIV screening and proposed solutions. Nursing Outlook, 60, 72-80. doi:10.1016/j.outlook.2011.06.002

De Santis, J. P. (2013). Depression among patients with HIV infection: Implications for case managers. Care Management, 14-17.

De Santis, J. P., Arcia, A., Vermeesch, A., \& Gattamorta, K. A. (2011). Using structural equation modeling to identify predictors of sexual behaviors among Hispanic men who have sex with men. Nursing Clinics of North America, 46, 233-248. doi:10.1016/j.cnur.2011.02.010

De Santis, J. P., \& Barroso, S. (2011). Living in silence: A grounded theory study of vulnerability in the context of HIV infection. Issues in Mental Health Nursing, 32(6), 345-354. doi:10.3109/01612840.2010.550018 
De Santis, J. P., Gonzalez-Guarda, R., Provencio-Vasquez, E., \& Deleon, D. A. (2014). The tangled branches (las ramas enredadas): Sexual risk, substance abuse, and intimate partner violence among Hispanic men who have sex with men. Journal of Transcultural Nursing, 25(1), 23-32. doi:10.1177/1043659613504110

De Santis, J. P., Gonzalez-Guarda, R. M., \& Vasquez, E. P. (2012). Psychosocial and cultural correlates of depression among Hispanic men with HIV infection: A pilot study. Journal of Psychiatric and Mental Health Nursing, 19, 860-869. doi:10.1111/j.1365-2850.2011.01865.x

De Santis, J. P., Martin Layerla, D., Barroso, S., Gattamorta, K. A., Sanchez, M., \& Prado, G. J. (2012). Predictors of eating attitudes and behaviors among gay Hispanic men. Archives of Psychiatric Nursing, 26(2), 111-126. doi:10.1016/j.apnu.2011.06.003

De Santis, J. P., Provencio-Vasquez, E., Weidel, J. J., Watson, S., \& Sanchez, M. (2009). A comparison of depressive symptoms, self-esteem, and sexual behaviors between foreign-born and U.S.-born Hispanic men who have sex with men: Implications for HIV prevention. Hispanic Health Care International, 7(2), 8087. doi:10.1891/1540-4153.7.2.80

De Santis, J. P., Valdes, B., Provencio-Vasquez, E., \& Gattamorta, K. A. (2015). A comparison by sexual orientation of sexual health and sexual behaviors among Hispanic men. Sexuality Research and Social Policy, 12(1), 15-23. doi:10.1007/s13178-014-0170-9

De Santis, J. P., Valdes, B., Provencio-Vasquez, E., Patsdaughter, C. A., \& Gattamorta, K. A. (2014). A comparison of substance use behaviors of Hispanic men by sexual orientation. Nursing and Health, 2(1), 9-17. doi:10.13189/nh.2014.020102

De Santis, J. P., \& Vasquez, E. P. (2010). An appraisal of the factors influencing human sexuality research in nursing. Nursing Forum, 45(3), 174-184. doi:10.1111/j.1744-6198.2010.00187.x

De Santis, J. P., Vasquez, E., Deleon D., \& Gonzalez-Guarda, R. (2012). Relationships as risk for HIV infection: High risk sex, substance abuse, and violence among Hispanic men who have sex with men. Horizonte de Enfermeria, 23(1), 27-39.

DeVellis, R. F. (2003). Scale Development: Theory and Applications. (2 ${ }^{\text {nd }}$ ed.). Thousand Oaks, CA: Sage.

Díaz, R. M., Ayala, G., \& Bein, E. (2004). Sexual risk as an outcome of social oppression: Data from a probability sample of Latino gay men in three US cities. Cultural Diversity \& Ethnic Minority Psychology, 10(3), 255-267. 
Faul, F., Erdfelder, E., Buchner, A., \& Lang, A. G. (2009). Statistical power analyses using G*Power 3.1: Tests for correlation and regression analyses. Behavior Research Methods, 41, 1149-1160.

Faul, F., Erdfelder, E., Lang, A. G., \& Buchner, A. (2007). G*Power 3: A flexible statistical power analysis program for the social, behavioral, and biomedical sciences. Behavior Research Methods, 39, 175-191.

Fernandez, M. I., Bowen, G. S., Warren, J. C., Ibanez, G. E., Hernandez, N., Harper, G. W., \& Prado, G. (2007, January). Crystal methamphetamine: A source of added sexual risk for Hispanic men who have sex with men. Drug \& Alcohol Dependence, 86, 245-252. doi:10.1016/j.drugalcdep.2006.06.016

Fernandez, M. I., Jacobs, R. J., Warren, J. C., Sanchez, J., \& Bowen, G. S. (2009). Drug use and Hispanic men who have sex with men in South Florida: Implications for intervention development. AIDS Education \& Prevention, 21, 45-60. doi:0.1521/aeap.2009.21.5_supp.45

Gasiorowicz, M., Llanas, M. R., DiFranceisco, W., Benotsch, E. G., Brondino, M. J., Catz, S. L., Hoxie, N. J., ...Vergeront, J. M. (2005). Reductions in transmission risk behaviors in HIV-positive clients receiving prevention case management services: Findings from a community demonstration project. AIDS Education and Prevention, 17, 40-52. doi:10.1521/aeap.17.2.40.58694

Gonzalez-Guarda, R. M., Florom-Smith, A. L., \& Thomas, T. (2011). A syndemic model of substance abuse, intimate partner violence, HIV infection, and mental health among Hispanics. Public Health Nursing, 28(4), 366-378. doi:10.1111/j.15251446.2010.00928.x

Gonzalez-Guarda, R. M., Ortega, J., Vazquez, E. P., \& De Santis, J. (2010). La mancha negra: Substance abuse, violence, and sexual risks among Hispanic males. Western Journal of Nursing Research, 32(1), 128-148. doi:10.1177/0193945909343594

Grov, C., Golub, S. A., Parsons, J. T., Brennan, M., \& Karpiak, S. E. (2010). Loneliness and HIV-related stigma explain depression among older HIV-positive adults. AIDS Care, 22(5), 630-639. doi:10.1080/09540120903280901

Guarnero, P. A. (2007). Family and community influences on the social and sexual lives of Latino gay men. Journal of Transcultural Nursing, 18(1), 12-18. doi:10.1177/1043659606294195

Guarnero, P. A. (2013). Latino young men and health promotion, emerging adulthood, and acculturation: A qualitative exploration. Issues in Mental Health Nursing, 34, 796-802. doi:10.3109/01612840.2013.825680 
Halkitis, P. N., Kupprat, S. A., Hampton, M. B., Perez-Figueroa, R., Kindon, M., Eddy, J. A., \& Ompad, D. C. (2013). Evidence for a syndemic in aging HIV-positive gay, bisexual, and other MSM: Implications for a holistic approach to prevention and health care. Annals of Anthropological Practice, 36(2), 365-386.

doi:10.IIII/napa.12009

Hall, H. I., Byers, R. H., Ling, Q., \& Espinoza, L. (2007, June). Racial/Ethnic and age disparities in HIV prevalence and disease progression among men who have sex with men in the United States. American Journal of Public Health, 97(6), 10601066. doi:10.2105/AJPH.2006.087551

Hall, H. I., Song, R., Rhodes, P., Prejean, J., An, Q., Lee, L. M., Karon, J., ...Janssen, R. S. [HIV Incidence Surveillance Group]. (2008). Estimation of HIV incidence in the United States. The Journal of the American Medical Association (JAMA), 300(5), 520-529. doi:10.1001/jama.300.5.520

Hawes, S. M., \& Berkley-Patton, J. Y. (2014). Religiosity and risky sexual behaviors among an African American church based population. Journal of Religion and Health, 53(2), 469-482. doi:10.1007/s10943-012-9651-4

Houston, E., Sandfort, T., Dolezal, C., \& Carballo-Dieguez, A. (2012). Depressive symptoms among MSM who engage in bareback sex: Mood matter? AIDS and Behavior, 16, 2209-2215. doi:10.1007/s10461-012-0156-7

Hubach, R., Dodge, B., Li, M. J., Schick, V., Herbenick, D., Ramos, W. D., ...Reece, M. (2015). Loneliness, HIV-related stigma, and condom use among a predominantly rural sample of HIV-positive men who have sex with men (MSM). AIDS Education \& Prevention, 27(1), 72-83. doi:10.1521/aeap.2015.27.1.72

Hughes, M. (2009). Lesbian and gay people's concerns about ageing and accessing services. Australian Social Work, 62(2), 186-201.

doi:10.1080/03124070902748878

Jacobs, R. J., Rasmussen, L. A., \& Hohman, M. M. (2008). The social support needs of older lesbians, gay men, and bisexuals. Journal of Gay \& Lesbian Social Services, 9(1), 1-30. doi:10.1300/J041v09n01_01

Jarvis, C. (2012). Physical examination \& health assessment. St. Louis, MO: Elsevier Saunders.

Johnson, C., Heckman, T., Hansen, N., Kochman, A., \& Sikkema, K. (2009). Adherence to antiretroviral medication in older adults living with HIV/AIDS: A comparison of alternative models. AIDS Care, 21(5), 541-551. doi:10.1080/095401208023802385611 
Kelly, J. A., Amirkhanian, Y. A., Seal, D. W., Galletly, C. M., DiFranceisco, W., Glasman, L., ...Rosado, N. (2010). Levels and predictors of sexual HIV risk in social networks of men who have sex with men in the Midwest. AIDS Education and Prevention, 22(6), 483-495. doi:10.1521/aeap.2010.22.6.483

Kertzner, R. M. (2014). The adult life course and homosexual identity in midlife gay men. Annual Review of Sex Research, 12(1), 75-92. doi:10.1080/10532528.2001.10559794

Klein, H. (2011). Using a Syndemics Theory approach to studying HIV risk taking in a population of men who use the Internet to find partners for unprotected sex. American Journal of Men's Health, 5(6), 466-476. doi:10.1177/1557988311398472

Kurtz, S. P., Buttram, M. E., Surratt, H. L., \& Stall, R. D. (2012). Resilience, syndemic factors, and serosorting behaviors among HIV-positive and HIV-negative substance-using MSM. AIDS Education and Prevention, 24(3), 193-205. doi:10.1521/aeap.2012.24.3.193

Lauby, J. L., Marks, G., Bingham, T., Liu, K., Liau, A., Stueve, A., \& Millett, G. A. (2012). Having supportive social relationships is associated with reduced risk of unrecognized HIV infection among Black and Latino men who have sex with men. AIDS and Behavior, 16, 508-516. doi:10.1007/s10461-011-0002-3

Lieb, S., Arons, P., Thompson, D. R., Santana, A. M., Liberti, T. M., Maddox, L., Bush, T., \& Fallon, S. J. (2006). Men who have sex with men: Racial/ethnic disparities in estimated HIV/AIDS prevalence at the state and county level, Florida. AIDS Behavior, 8, 1-8. doi:10.1007/s10461-008-9411-3

Lim, F. A., Brown, D. V., \& Jones, H. (2013). Lesbian, gay, bisexual, and transgender health: Fundamentals for nursing education. Journal of Nursing Education, 52(4), 198-203. doi:10.3928/01484834-20130311-02

Liu, R. T., \& Mustanski, B. (2012). Suicidal idealation and self-harm in lesbian, gay, bisexual, and transgender youth. American Journal of Preventative Medicine, 42(3), 221-228. doi:10.1016/j.amepre.2011.10.023

Lo, S. C., Reisen, C. A., Poppen, P. J., Bianchi, F. T., \& Zea, M. C. (2011). Cultural beliefs, partner characteristics, communication, and sexual risk among Latino MSM. AIDS Behavior, 15, 613-620. doi:10.1007/s10461-010-9760-6

Louis Calder Memorial Library. (2012). The Louis Calder Memorial Library brochure. Retrieved from http://calder.med.miami.edu/electronic_databases.html\#p1 
MacCallum, R. C., Browne, M. W., \& Cai, L. (2006). Testing differences between covariance structure models: Power analysis and null hypothesis. Psychological Methods, 11(1), 19-35. doi:10.1037/1082-989X.11.1.19; 10.1037/1082989X.11.1.19.supp

Maisto, S. A., Palfai, P., Vanable, P. A., Heath, J., \& Woolf-King, S. E. (2012). The effects of alcohol and sexual arousal on determinants of sexual risk in men who have sex with men. Archives of Sexual Behaviors, 41, 971-986. doi:10.1007/s10508-011-9846-X

Marin, G., \& Marin, B. V. (1991). Research with Hispanic Populations. Newbury Park, CA: Sage.

Martin, J. I., \& Knox, J. (1997). Loneliness and sexual risk behavior in gay men. Psychology Reports, 81(3), 815-825. doi:10.2466/pr0.1997.81.3.815

Meyer, M. A., \& Champion, D. (2008). Motivators of HIV risk-taking behavior of young gy latino men. Journal of the American Psychiatric Nurses Association, 14(4), 310-316. doi:10.1177/1078390308321926

Moeller, R. W., Halkitis, P. N., Surrence, K. (2011). The interplay of syndemic production and serosorting in drug-using gay and bisexual men. Journal of Gay \& Lesbian Social Services, 23(1), 89-106. doi:10.1080/10538720.2010.538007

Morris, C. for SAMHSA-HRSA Center for Integrated Health Solutions. (2012). Supporting clients in sexual health: A provider action brief. Retrieved from http://www.integration.samhsa.gov/health-wellness/wellnessstrategies/Sexual_Health_Provider_Action_Brief.pdf

Mustanski, B. \& Lui, R. T. (2013). A longitudinal study of predictors of suicide attempts among lesbians, gay, bisexual, and transgender youth. Archives of Sexual Behavior, 42, 437-448. doi:10.1007/s10508-012-0013-9

O’Cleirigh, C., Newcomb, M. E., Mayer, K. H., Skeer, M., Traeger, L., \& Safren, S. A. (2013). Moderate levels of depression predict sexual transmission risk in HIVinfected MSM: A longitudinal analysis of data from six sites involved in a "prevention for positives" study. AIDS and Behavior, 17, 1764-1769. doi:10.1007/s10461-013-0462-8

O’Donnell, L., Stueve, A., Joseph, H. A., \& Flores, S. (2014). Adapting the VOICES HIV behavioral intervention for Latino men who have sex with men. AIDS and Behavior, 18, 767-775. doi:10.1007/s10461-013-0653-3 
Office of Management and Budget (OMB). (1997, October). Revisions to the Standards for the Classification of Federal Data on Race and Ethnicity. Retrieved from http://www.whitehouse.gov/omb/fedreg_1997standards

Organization for Economic Cooperation and Development (OECD). (2013). OECD health data 2013: How does the United States compare? Retrieved from http://www.oecd.org/unitedstates/Briefing-Note-USA-2013.pdf

Ortman, J. M., \& Guarneri, C. E. (2009). United States population projections: 2000 to 2050. Retrieved from http://www.census.gov/population/www/projections/analytical-document09.pdf

Pallant, J. (2010). SPSS Survival Manual. ( $4^{\text {th }}$ ed.). NY, New York: McGraw-Hill Education.

Parsons, J. T., Grov, C., \& Golub, S. A. (2012). Sexual compulsivity, co-occurring psychological health problems, and HIV risk among gay and bisexual men: Further evidence of a syndemic. American Journal of Public Health, 102(1), 156162. doi:10.2105/AJPH.2011.300284

Passel, J. S. \& Cohn, D. (2008). U.S. Population projections: 2005-2050. Pew Research Center. Retrieved from http://www.pewhispanic.org/2008/02/11/us-populationprojections-2005-2050/

Polit, D. F., \& Beck, C. T. (2012). Nursing research: Generating and assessing evidence for nursing practice. ( ${ }^{\text {th }}$ ed.). Philadelphia, PA: Lippincott Williams \& Wilkins.

Potocky, M., Dodge, K., \& Greene, M. (2007). Bridging cultural chasms between providers and HIV-positive Haitians in Palm Beach County, Florida. Journal of Health Care for the Poor and Underserved, 18(3), 105-117.

Price, E. (2005). All but invisible: Older gay men and lesbians. Nursing Older People, 17(4), 16-18.

Radloff, L. S. (1977). The CES-D scale: A self-report depression scale for research in the general population. Applied Psychological Measurement, 1(5), 385-401.

Ramirez-Valles, J. (2007). Interventions for Latino gay men. American Journal of Preventive Medicine, 32(4S), S34-S35. doi:10.1016/j.amepre.2006.12.024

Rhodes, S. D., Hergenrather, K. C., Vissman, A. T., Stowers, J., Davis, A. B., Hannah, A., ...Marsiglia, F. F. (2011). Boys must be men, and men must have sex with women: A qualitative CBPR study to explore sexual risk among African American, Latino, and White gay men and MSM. American Journal of Men's Health, 5(2), 140-151. doi:10.1177/1557988310366298 
Rhodes, S. D., McCoy, T. P., Hergenrather, K. C., Vissman, A. T., Wolfson, M., Alonzo, J., ...Eng, E. (2012). Prevalence estimates of health risk behaviors of immigrant Latino men who have sex with men. The Journal of Rural Health, 28, 73-83. doi:10.1111/j.1748-0361.2011.00373.x

Russell, D., Peplau, L. A., \& Ferguson, M. L. (1978). Developing a measure of loneliness. Journal of Personality Assessment, 42(3), 290-294.

Russell, D., Peplau, L. A., \& Cutrona, C. E. (1980). The revised UCLA Loneliness Scale concurrent and discriminate validity evidence. Journal of Personality and Social Psychology, 39, 472-480.

Russell, D. W. (1996). UCLA Loneliness Scale (version 3): Reliability, validity, and factor structure. Journal of Personality Assessment, 66(1), 20-40.

Salkind, N. J. (2013). Statistics for people who (think they) hate statistics. ( $5^{\text {th }}$ ed.). Thousand Oaks, CA: Sage.

Sandfort, T. G. M., Melendez, R. M., \& Diaz, R. M. (2007). Gender nonconformity, homophobia, and mental distress in Latino gay and bisexual men. Journal of Sex Research, 44(2), 181-189. doi:10.1080/00224490701263819

Semple, S. J., Zians, J., Grant, I, \& Patterson, T. L. (2006). Methamphetamine use, impulsivity, and sexual risk behavior among HIV-positive men who have sex with men. Journal of Addictive Diseases, 25(4), 105-114.

Sikkema, K. J., Abler, L., Hansen, N. B., Wilson, P. A., Drabkin, A. S., Kochman, A., MacFarlane, ...Nazareth, W. (2014). Positive choices: Outcomes of a brief risk reduction intervention for newly HIV-diagnosed men who have sex with men. AIDS and Behavior, 18, 1808-1819. doi:10.1007/s10461-014-0782-3

Singer, M. (1996). A dose of drugs, a touch of violence, a case of AIDS: Conceptualizing the SAVA syndemic. Free Inquiry in Creative Sociology, 24(2), 99-110.

Singer, M. (2009). Introduction to Syndemics: A critical systems approach to public and community health. San Francisco, CA: Jossey-Bass.

Singer, M. C., Erickson, P. I., Badiane, L., Diaz, R., Ortiz, D., Abraham, T., \& Nicolaysen, A. M. (2006). Syndemics, sex and the city: Understanding sexually transmitted disease in social and cultural context. Social Science and Medicine, 63(8), pp. 2010-2021. doi:10.1016/j.socscimed.2006.05.012 
Skinta, M. D., Murphy, J. L., Paul, J. P., Schwarcz, S. K., \& Dilley, J. W. (2012). Thoughts, attitudes, and feelings of HIV-positive MSM associated with high transmission-risk sex. Health Education \& Behavior,39(3), 315-323. doi:10.1177/1090198111427390

Stall, R., Friedman, M., \& Catania, J. A. (2007). Interacting epidemics and gay men’s health: A theory of syndemic production among urban gay men. In R. J. Wolitski, R. Stall \& R. O. Valdiserri (Eds.), Unequal Opportunity: Health Disparities Affecting Gay and Bisexual Men in the United States, (Chapter 10). Oxford: UK, Oxford University Press. doi:10.1093/acprof:oso/9780195301533.003.0009

Stall, R., Mills, T. C., Williamson, J., Hart, T., Greenwood, G., Paul, J., ...Catania, J. A. (2003). Association of co-occurring psychosocial health problems and increased vulnerability to HIV/AIDS among urban men who have sex with men. American Journal of Public Health, 93(6), 939-942. doi:10.2105/AJPH.93.6.939

Substance Abuse and Mental Health Service Administration (SAMHSA). (2010). The national survey on drug use and health (NSDUH) report: Substance abuse among Hispanic adults. Retrieved from http://www.oas.samhsa.gov/2k10/184/HispanicAdults.pdf

Sullivan, G. M., \& Feinn, R. (2012). Using effect size-or why the $p$ value is not enough. Journal of Graduate Medical Education, 4(3), 279-282. doi:10.4300/JGME-D12-00156.1

Szapocznik, J., Kurtines, W. M., \& Fernandez, T. (1980). Bicultural involvement and adjustment in Hispanic-American youths. International Journal of Intercultural Relations, 4, 353-365.

The Florida Department of Health in Miami-Dade County. (2010). HIV surveillance. Retrieved from http://miamidade.floridahealth.gov/programs-andservices/infectious-disease-services/hiv-aids-services/hiv-surveillance.html

Toro-Alfonso, J., Varas-Díaz, N., \& Andújar-Bello, I. (2002). Evaluation of an HIV/AIDS prevention intervention targeting Latino gay men and men who have sex with men in Puerto Rico. AIDS Education and Prevention, 14(6), 445-456.

Torres, H. L., \& Gore-Felton, C. (2007). Compulsivity, substance use, and loneliness: The Loneliness and Sexual Risk Model (LSRM). Sexual Addition \& Compulsivity, 14(1), 63-75. doi:10.1080/10720160601150147

United States (US) Census Bureau. (2011, September 29). 2010 census shows white population growth fueled by Hispanics. Retrieved from http://www.census.gov/newsroom/releases/archives/2010_census/cb11cn184.html 
United States Census Bureau. (2011). Database: Same-sex couple household. Retrieved from http://www.census.gov/hhes/samesex/

Vance, D. E., McGuinness, T., Musgrove, K., Orel, N. A., \& Fazeli, P. L. (2011). Successful aging and the epidemiology of HIV . Clinical Interventions in Aging, 6, 181-192. doi:10.2147/cia.s14726

Vazquez, E. P., Gonzalez-Guarda, R. M., \& De Santis, J. P. (2011). Acculturation, depression, self-esteem, and substance abuse among Hispanic men. Issues in Mental Health Nursing, 32, 90-97. doi:10.3109/01612840.2010.528169

Vega, M. Y., Spieldenner, A. R., DeLeon, D., Nieto, B. X., \& Stroman, C. A. (2011). SOMOS: Evaluation of an HIV prevention intervention for Latino gay men. Health Education Research, 26(3), 407-418. doi:10.1093/her/cyq068

Waltz, C. F., Strickland, O. L., \& Lenz, E. R. (2010). Measurement in Nursing and Health Research. (4 ${ }^{\text {th }}$ ed.). New York, NY: Springer Publishing.

Weeks, D. G., Michela, J. L., Peplau, L. A., \& Bragg, M. E. (1980). Relation between loneliness and depression: A structural equation analysis. Journal of Personality and Social Psychology, 39, 1238-1244.

Well, B. E., Golub, S. A., \& Parsons, J. T. (2011). An integrated theoretical approach to substance use and risky sexual behavior among men who have sex with men. AIDS Behavior, 15, 509-520. doi:10.1007/s10461-010-9767-z

Wisconsin HIV Prevention Evaluation Work Group. (2000). Behavioral risk assessment tool (BRAT). Retrieved from http://www.cdc.gov/hiv/topics/evaluation/health_depts/guidance/strathandbook/pdf/Appendix87.pdf

Wrubel, J., Stumbo, S., \& Johnson, M. (2008). Antiretroviral medication support practices among partners of men who have sex with men: A qualitative study. AIDS Patient Care and STDs, 22(11), 851-858. doi:10.1089apc.2008.0037.

Zimet, G. D., Dahlem, N. W., Zimet, S. G., \& Farley, G. K. (1988). The multidimensional scale of perceived social support (MSPSS). Journal of Personality Assessment, 52(1), 30-41.

Zuckerman, M., \& Lubin, B. (1965). The multiple affect adjective checklist (MAACL). Retrieved from https://ubir.buffalo.edu/xmlui/handle/10477/1899 


\section{APPENDIX A}

Demographic Questionnaire: English

1) Please tell me where you were born (country of birth).

\begin{tabular}{|c|c|c|c|c|c|c|}
\hline United States & 0 & Costa Rica & 0 & Honduras & 0 & Puerto Rico \\
\hline Argentina & ○ & Cuba & 0 & Mexico & 0 & Uruguay \\
\hline Bolivia & ○ & Dominican Republic & 0 & Nicaragua & 0 & Venezuela \\
\hline Brazil & ○ & Ecuador & 0 & Panama & & \\
\hline Chile & ○ & El Salvador & 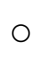 & Paraguay & 0 & Other \\
\hline Colombia & 0 & Guatemala & O & Peru & & \\
\hline
\end{tabular}
(specify:

1a. Years living in U.S.

2) What is your current relationship status?
- Single
○ In a relationship, not legally married
○ Married
○ Divorced
○ Separated
○ Widowed

3) Are you currently living with your spouse or partner?
○ YES
○ NO
○ Not Applicable

4) Your current partner is: $\circ$ Male $\circ$ Female $\circ$ Not Applicable

4a. How do you identify yourself?

$\circ$ Heterosexual $\bigcirc$ Homosexual o Bisexual oPrefer not to answer

5) How many children do you have?

○ None (SKIP to 6)

5a. Do any of your children live in another country? ○ YES O NO (SKIP to 6)

5a1. If yes, give their ages, country where they live, and number of years you have lived apart (in another country) from them. List from youngest to oldest. If there are more than 4 children living in another country, bubble here: $\bigcirc$

\begin{tabular}{|c|c|c|}
\hline Child 1Age & Country & Years apart \\
\hline Child 2Age & Country & Years apart \\
\hline Child 3Age & Country & Years apart \\
\hline Child 4Age & Countr & Years apart \\
\hline
\end{tabular}


6) What is your religion?

\begin{tabular}{|c|c|c|c|c|c|}
\hline Baptist & Jehovah's & & Presbyte & & \\
\hline Christian & Jewish & 0 & Protesta & & \\
\hline Episcopalian & Methodist & 0 & Roman & & \\
\hline \multicolumn{2}{|c|}{$\begin{array}{l}\text { Evangelist/Pentecostal } \\
\text { Other Christian (Specify }\end{array}$} & 0 & Muslim & 0 & None \\
\hline
\end{tabular}

7) How often do you attend religious services? Would you say...

$\circ$ More than once a week $\quad \circ$ Less than once a month

○ Weekly $\bigcirc$ Only on special days

$\circ$ Monthly (1+) $\quad$ Not at all

8) Do you consider yourself?

$\circ$ Not religious $\bigcirc$ Somewhat religious $\bigcirc$ Very religious

9) How strongly do the beliefs of your religion influence your life?
$\circ$ Not at all
○ Somewhat
$\circ$ Very much
○ Not Applicable

10) How many years of education you have completed?

11) Are you currently employed? $\quad \circ$ YES (SKIP to 12) $\circ$ NO

11a. If no, when was the last time you had a job?

oMore than 1 year ago oLess than 1 year ago oNever been employed

12) Last month, what was the total amount of money you and your family lived on, including public assistance (after taxes)?

13) How many people in this country lived from this money?

14) Do you have health insurance? $\quad \circ$ YES $\quad$ NO

15) How do you usually pay for your own health care? (Bubble only one)
- Private Insurance Plan (not provided at work)
- Medicare
- Private Insurance Plan (provided at work)
- Medicaid
- Out of Pocket
- Other (Specify
○ Don't Pay

16) Where do you usually go when you are sick or want advice about your health? (Bubble all that apply)

\begin{tabular}{lllll}
$\circ$ & Clinic & $\circ$ & Nurse Practitioner & $\circ$ Doctor’s Office \\
$\circ$ & Emergency Room & $\circ$ & “Curandero” & \\
$\circ$ & Family Member, Friend, or Neighbor & \\
$\circ$ & Other (Specify & \\
\hline
\end{tabular}


17) Do you have a regular doctor or healthcare provider? $\quad \circ$ YES $\quad \circ$ NO

18) When was the last time you saw your doctor or healthcare provider?

Month/Year

19) How many times were you in the emergency room in the past three months for your health problems?

20) How would you describe your health in the past three months?
○ Poor
$\circ$ Fair
○ Good
- Very Good

21) Have you ever been tested for HIV?

$\circ$ Yes $\quad \circ$ No

22) What was the result of your most recent HIV test?
$\circ$ Positive
$\circ$ Negative
○ Don’t know

23) If you have HIV, how long have you been living with HIV?

24) Are you receiving medical care for your HIV?
$\circ$ Yes
○ No

25) At any time in your life, have you considered suicide?

○ Yes o No

26) When was the last time you considered suicide?

27) At any time in your life, have you attempted suicide?

○ Yes o No

28) When was the last time you attempted suicide?

29) Are you currently receiving any mental health care?

○ Yes o No

30) If yes, what services? 


\section{APPENDIX B}

Demographic Questionnaire: Spanish

1) Favor indicar dónde nació. (País de nacimiento).

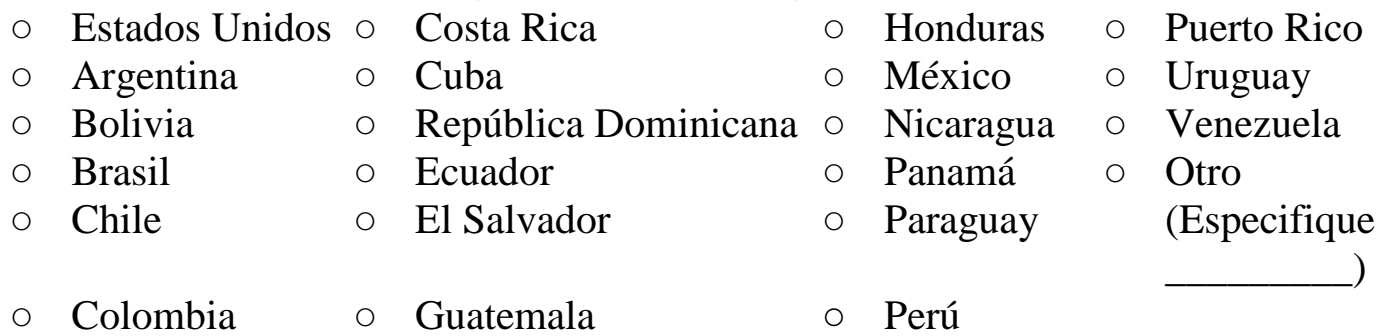

1a. Años en los Estados Unidos

2.) ¿Cuál es su estado civil actual?

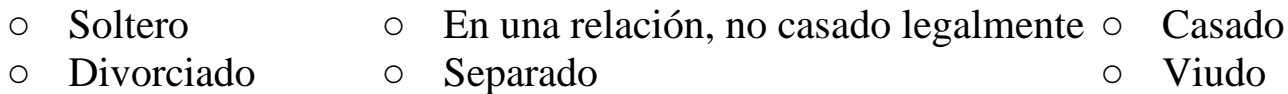

3) ¿Actualmente convive con su esposo(a) o pareja?
○ SÍ
○ NO
- No Aplica

4) Su pareja actual es: $\bigcirc$ Femenina $\bigcirc$ Masculino $\bigcirc$ No Aplica

4a. ¿Cómo usted se identifica?

$\circ$ Heterosexual $\bigcirc$ Homosexual $\bigcirc$ Bisexual $\bigcirc$ Prefiere no contestar

5) ¿Cuántos hijos tiene?

$\circ \quad$ Ninguno (SKIP to 6)

5a. ¿Alguno de sus hijos viven en otro país?
○ SÍ
$\circ \quad \mathrm{NO}$ (SKIP to 6)

5a1. Si contesto SÍ, escriba sus edades, país donde viven y el número de anos que usted vive separado de ellos (en otro país). Haga la lista desde el más pequeño de edad al más grande. Si tiene más de 4 hijos viviendo en otro país, marque aquí: o

\begin{tabular}{|c|c|c|}
\hline Hijo(a) 1 Edad & País & Años separados \\
\hline Hijo(a) 2 Edad & País & Años separados \\
\hline Hijo(a) 3 Edad & País & Años separados \\
\hline Hijo(a) 4 Edad & País & Años separados \\
\hline
\end{tabular}


6) ¿ ¿Cuál es su religión?

\begin{tabular}{|c|c|c|c|}
\hline Bautista & Testigo de Jehová & 0 & Presbiteriano \\
\hline Cristiano & Judío & 0 & Protestante \\
\hline Episcopal & Metodista & o & Católico Romano \\
\hline Evangélico/Pentecostal & Musulmán & 0 & Ninguno \\
\hline
\end{tabular}

7) ¿Qué tan frecuente asiste a servicios religiosos? Diría..

- Más de una vez a la semana $\quad 0$ Menos de una vez al mes

- Semanal

○ Mensual (1+)

- Solamente en ocasiones especiales

- Nunca

8) ¿ ¿Usted se considera...? (INTERVIEWER: Read all choices)

$\circ$ No religioso $\bigcirc$ Un poco religioso $\bigcirc$ Muy religioso

9) ¿Qué tan importante ha sido la influencia de la religión en su vida?

(ENTREVISTADOR: Lea todas las opciones)

$\circ$ Nada $\quad \circ$ Un poco $\bigcirc$ Muchísimo $\bigcirc$ No Aplica

10) ¿Cuántos años de educación ha completado?

11) ¿Está actualmente empleado? $\quad \circ \quad$ Sí (PASE a 12) $\circ \quad$ NO

11a) Si contesta "NO”, ¿cuándo fue la última vez que tuvo un trabajo?

○ Hace más de un año $\quad \circ$ Hace menos de un año

- Nunca ha tenido empleo

12) El mes pasado, ¿Cuál fue la cantidad total de dinero con la cual usted y su familia vivió, incluyendo asistencia pública?

13) ¿Cuántas personas en este país vivieron de ese dinero?

14) ¿Tiene usted seguro médico? $\quad \circ$ SÍ $\quad \circ \quad$ NO

15) ¿Cómo usted usualmente paga por su propio servicio de salud? (Marque sólo una)

$\circ$ Plan de seguro privado (no provisto por su empleo) $\bigcirc$ Medicare

$\circ$ Plan de seguro privado (provisto por su empleo) $\quad$ Con su propio

$\circ$ Medicaid

$\circ$ Otro (Especifique __ $\quad \circ \quad$ No paga 
16) ¿Dónde va usted usualmente cuando esta enfermo o necesita consejo sobre su salud? (Marque todas las que correspondan)
○ Clínica
○ Enfermera especializada de grado avanzado
- Oficina del Doctor
- Sala de Emergencia
- "Curandero"
- Un familiar, amigo, o vecino
- Otro (Especifique:

17) ¿Tiene usted un doctor regular o un proveedor de salud? $\quad \circ \quad$ Sí $\quad \circ \quad$ No

18) ¿Cuándo fue la última vez que visitó su médico o proveedor de salud?

Mes/Año

19) ¿Cuántas veces en los últimos tres meses visitó la sala de emergencia por sus problemas de salud?

20) ¿Cómo describe usted su salud en los últimos tres meses?
○ Mala
○ Más o Menos
○ Buena
○ Muy Buena

21) ¿Te has hecho alguna vez la prueba del VIH? $\bigcirc \mathrm{Si} \quad \circ \quad$ No

22) ¿Cuál fue el resultado de tu última prueba del VIH?
$\circ$ Positiva
$\circ$ Negativa
$\circ$ Desconocido

23) Si usted tiene VIH, cuanto tiempo ha vivido con VIH?

○ No aplica

24) Esta recibiendo tratamiento médico para el VIH?
$\circ \mathrm{Si}$
○ No
○ No aplica

25) ¿En algún momento en su vida, ha considerado el suicidio?
$\circ \mathrm{Si}$
○ No
- No aplica

26) ¿Cuándo fue la ultima vez que usted considero suicidarse?

$\circ \quad$ No aplica

27) ¿En algún momento en su vida usted ha tratado de suicidarse?

$\circ \mathrm{Si} \quad \circ \quad$ No

28) ¿Cuándo fue la última vez que trato de suicidarse?

$\circ$ No aplica

29) ¿Está usted recibiendo alguna atención de la salud mental?
○ $\mathrm{Si}$
$\circ$ No

30) En caso afirmativo, ¿qué servicios? 


\section{APPENDIX C}

MSPSS, Zimet, Dahlem, Zimet, \& Farley, 1988: English

INSTRUCTIONS : We are interested in knowing more about what type of support you have around you when you get sick.

For each of the next 12 statements, indicate how you feel about each statement by selecting [agree], [disagree], or [neither agree nor disagree] with the statement. Circle response.

$\begin{array}{ll}1= & \text { Very Strongly Disagree } \\ 2= & \text { Strongly Disagree } \\ 3= & \text { Mildly Disagree } \\ 4= & \text { Neither } \\ 5= & \text { Mildly Agree } \\ 6= & \text { Strongly Agree } \\ 7= & \text { Very strongly Agree }\end{array}$

1) There is a special person who is around when I am in $\begin{array}{lllllll}1 & 2 & 3 & 4 & 5 & 6 & 7\end{array}$ need.

2) There is a special person with whom I can share my $\quad \begin{array}{lllllll}1 & 2 & 3 & 4 & 5 & 6 & 7\end{array}$ joys and sorrows.

3) My family really tries to help me. $\quad 1 \quad 2 \quad 3 \quad 4 \quad 567$

4) I get the emotional help and support I need from my $\quad \begin{array}{lllllll}1 & 2 & 3 & 4 & 5 & 6 & 7\end{array}$ family.

5) I have a special person who is a real source of comfort to me.

6) My friends really try to help me.

$\begin{array}{lllllll}1 & 2 & 3 & 4 & 5 & 6 & 7\end{array}$

7) I can count on my friends when things go wrong. $\quad \begin{array}{lllllll}1 & 2 & 3 & 4 & 5 & 6 & 7\end{array}$

8) I can talk about my problems with my family. $\quad \begin{array}{lllllll}2 & 2 & 3 & 4 & 5 & 6 & 7\end{array}$

9) I have friends with whom I can share my joys and $\begin{array}{lllllll}1 & 2 & 3 & 4 & 5 & 6\end{array}$ sorrows.

10) There is a special person in my life who cares about $\begin{array}{lllllll}1 & 2 & 3 & 4 & 5 & 6 & 7\end{array}$ my feelings.

11) My family is willing to help me make decisions. $\quad \begin{array}{lllllll}1 & 2 & 3 & 4 & 5 & 6 & 7\end{array}$

12) I can talk about my problems with my friends. $\quad \begin{array}{lllllll}2 & 2 & 3 & 4 & 5 & 6 & 7\end{array}$ 


\section{APPENDIX D}

MSPSS, Zimet, Dahlem, Zimet, \& Farley, 1988: Spanish

INSTRUCCIONES : Estamos interesados en saber más sobre el tipo de ayuda que usted tiene a su alrededor cuando se enferma.

Para cada de las 12 declaraciones siguientes, indique cómo usted se siente sobre cada declaración y elegir [de acuerdo], [desacuerdo], o [ni de acuerdo o desacuerdo] con la declaración.

Circule la respuesta.

$1=\quad$ Muy fuertemente desacuerdo

$2=$ Fuertemente desacuerdo

$3=\quad$ Ligero desacuerdo

$4=\quad$ Ni de acuerdo ni de desacuerdo

$5=$ Ligeramente de acuerdo

$6=$ Fuertemente de acuerdo

$7=\quad$ Muy fuertemente de acuerdo

1) Hay una persona especial que esta cerca cuando tengo $1 \begin{array}{llllll}2 & 2 & 4 & 5 & 6 & 7\end{array}$ necesidad.

2) Hay una persona especial con quien puedo compartir mis $\quad \begin{array}{lllllll}1 & 2 & 3 & 4 & 5 & 6 & 7\end{array}$ alegrías y dolores.

3) Mi familia realmente me trata de ayudar. $\quad \begin{array}{lllllll}2 & 3 & 4 & 5 & 6 & 7\end{array}$

4) Yo recibo el apoyo y ayuda emocional que necesito $\quad \begin{array}{lllllll}1 & 2 & 3 & 4 & 5 & 6 & 7\end{array}$ de mi familia.

5) Tengo una persona especial que es una verdadera $\quad \begin{array}{lllllll}1 & 2 & 3 & 4 & 5 & 6 & 7\end{array}$ fuente de consuelo para mí.

6) Mis amigos realmente tratan de ayudarme. $\quad \begin{array}{lllllll}2 & 3 & 4 & 5 & 6 & 7\end{array}$

7) Puedo contar en mis amigos cuando las cosas van mal $1 \begin{array}{llllll}1 & 2 & 3 & 4 & 5 & 6\end{array}$

8) Puedo hablar de mis problemas con mi familia. $\quad \begin{array}{lllllll}2 & 2 & 3 & 4 & 5 & 6 & 7\end{array}$

9) Tengo amigos con quienes puedo compartir mis $\quad \begin{array}{lllllll}1 & 2 & 3 & 4 & 5 & 6 & 7\end{array}$ alegrías y dolores.

10) Hay una persona especial en mi vida que le importa $\quad \begin{array}{lllllll}1 & 2 & 3 & 4 & 5 & 6 & 7\end{array}$ mis sentimientos.

11) Mi familia está dispuesta a ayudarme a tomar $\quad \begin{array}{lllllll}1 & 2 & 3 & 4 & 5 & 6 & 7\end{array}$ decisiones.

12) Puedo hablar de mis problemas con mis amigos. $\quad \begin{array}{lllllll}1 & 2 & 3 & 4 & 5 & 6 & 7\end{array}$ 


\section{APPENDIX E}

UCLA Loneliness Scale, Russell, Peplau, \& Ferguson, 1978: English

O indicates "I often feel this way"

S indicates "I sometimes feel this way"

$\mathrm{R}$ indicates "I rarely feel this way"

$\mathrm{N}$ indicates "I never feel this way"

1) I am unhappy doing so many things alone $\quad \mathrm{O} S \mathrm{~S} \quad \mathrm{~N}$

2) I have nobody to talk to $\quad \mathrm{O} S \mathrm{~S} N$

3) I cannot tolerate being so alone $\quad \mathrm{O} \quad \mathrm{S} \quad \mathrm{R} \quad \mathrm{N}$

4) I lack companionship $\quad \mathrm{O} S \mathrm{~S} N$

5) I feel as if nobody really understands me $\quad O \quad S \quad R \quad N$

6) I find myself waiting for people to call or write $\quad O \quad S \quad R \quad N$

7) There is no one I can turn to $\quad \mathrm{O} S \mathrm{~S} N$

8) I am no longer close to anyone $\quad \mathrm{O} S \mathrm{~S} N$

9) My interests and ideas are not shared by those around me $\quad \mathrm{O} \quad \mathrm{S} \quad \mathrm{R} \quad \mathrm{N}$

10) I feel left out $\quad O \quad S \quad R \quad N$

11) I feel completely alone $\quad \mathrm{O} \quad \mathrm{S} \quad \mathrm{R} \quad \mathrm{N}$

12) I am unable to reach out and communicate with those around me $\mathrm{O} \quad \mathrm{S} \quad \mathrm{R} \quad \mathrm{N}$

13) My social relationships are superficial $\quad O \quad S \quad R \quad N$

14) I feel starved for company $\quad \mathrm{O} \quad \mathrm{S} \quad \mathrm{R} \quad \mathrm{N}$

15) No one really knows me well $\quad \mathrm{O} \quad \mathrm{S} \quad \mathrm{R} \quad \mathrm{N}$

16) I feel isolated from others $\quad \mathrm{O} \quad \mathrm{S} \quad \mathrm{R} \quad \mathrm{N}$

17) I am unhappy being so withdrawn $\quad O \quad S \quad R \quad N$

18) It is difficult for me to make friends $\quad O \quad S \quad R \quad N$

19) I feel shut out and excluded by others $\quad \mathrm{O} \quad \mathrm{S} \quad \mathrm{R} \quad \mathrm{N}$

20) People are around me but not with me $\quad \mathrm{O} \quad \mathrm{S} \quad \mathrm{R} \quad \mathrm{N}$ 


\section{APPENDIX F}

UCLA Loneliness Scale, Russell, Peplau, \& Ferguson, 1978: Spanish

$\mathrm{O}$ indica "A menudo me siento de esta manera"

$\mathrm{S}$ indica "A veces me siento de esta manera"

$\mathrm{R}$ indica "Rara vez me siento de esta manera"

$\mathrm{N}$ indica "Nunca me siento de esta manera"

1) Me siento infeliz por star haciendo tantas cosas solo

O

2) No tengo a nadie con quien hablar

O $\quad$ S $\quad \mathrm{R} \quad \mathrm{N}$

3) No puedo tolerar estar tan solo

O $\quad$ S $\quad \mathrm{R} \quad \mathrm{N}$

4) Me falta compañia

O

5) Siento que nadie realmente me comprenden

O $\quad$ S $\quad \mathrm{R} \quad \mathrm{N}$

6) Me encuentro esperando por personas que me llamen o escriban

O $\quad S \quad R \quad N$

7) No tengo a nadie con quien pueda contar

O

8) Ya no tengo a nadie cercano

$\begin{array}{llll}\mathrm{O} & \mathrm{S} & \mathrm{R} & \mathrm{N}\end{array}$

9) No tengo nada en común con los que me rodean

O $\quad$ S $\quad \mathrm{R} \quad \mathrm{N}$

10) Me siento excluido

O

11) Me siento completamente solo

O $\quad$ S $\quad \mathrm{R} \quad \mathrm{N}$

12) Soy incapaz de contactar y comunicarme con la gente

O $\quad S \quad R \quad N$ que me rodea

13) Mis relaciones sociales son superficiales

O

14) Me siento hambriento de compañia

O $\quad S \quad R \quad N$

15) Nadie me conoce bien

O

16) Me siento aislado de los demás

O

17) Me siento infeliz por star tan aislado

O $\quad \mathrm{S} \quad \mathrm{R} \quad \mathrm{N}$

18) Encuentro dificil hacer amigos

O S R N

19) Me siento excluido y olvidado por los demás

O $\quad$ S $\quad$ R $\quad \mathrm{N}$

20) La gente está a mi alrededor pero no conmigo

O $\quad S \quad R \quad N$ 


\section{APPENDIX G}

CES-Depression Scale (CES-D), Radloff, 1977: English

INSTRUCTIONS: Please answer how you have felt during the past week.

\begin{tabular}{|c|c|c|c|c|}
\hline $\begin{array}{l}\text { During the past week: } \\
\text { (Bubble only one response to the following } \\
\text { questions) }\end{array}$ & $\begin{array}{c}\text { Rarely } \\
\text { or } \\
\text { None } \\
\text { of the } \\
\text { time } \\
\text { (less } \\
\text { than } 1 \\
\text { day) }\end{array}$ & $\begin{array}{c}\text { Some } \\
\text { or a } \\
\text { little } \\
\text { of the } \\
\text { time } \\
\text { (1-2 } \\
\text { days) }\end{array}$ & $\begin{array}{c}\text { Occasional } \\
\text { ly or a } \\
\text { moderate } \\
\text { amount of } \\
\text { time } \\
\text { (3-4 days) }\end{array}$ & $\begin{array}{c}\text { Most } \\
\text { or all } \\
\text { the } \\
\text { time } \\
\text { (5-7 } \\
\text { days) }\end{array}$ \\
\hline $\begin{array}{l}\text { 1) I was bothered by things that usually } \\
\text { don't bother me }\end{array}$ & O & O & $\circ$ & ○ \\
\hline $\begin{array}{l}\text { 2) I did not feel like eating; my appetite } \\
\text { was poor }\end{array}$ & ○ & ○ & o & $\circ$ \\
\hline $\begin{array}{l}\text { 3) I felt that I could not shake off the } \\
\text { blues even with help from my family } \\
\text { or friends }\end{array}$ & ○ & O & ० & ○ \\
\hline $\begin{array}{l}\text { I) I felt that I was just as good as other } \\
\text { people }\end{array}$ & $\circ$ & O & $\circ$ & O \\
\hline $\begin{array}{l}\text { 5) I had trouble keeping my mind on } \\
\text { what I was doing }\end{array}$ & $\circ$ & ○ & $\circ$ & O \\
\hline 6) I felt depressed & $\circ$ & O & $\circ$ & $\circ$ \\
\hline $\begin{array}{l}\text { 7) I felt that everything I did was an } \\
\text { effort }\end{array}$ & $\circ$ & ○ & $\circ$ & O \\
\hline 8) I felt hopeful about the future & O & O & $\circ$ & $\circ$ \\
\hline 9) I thought my life had been a failure & O & $\mathrm{O}$ & $\circ$ & $\circ$ \\
\hline 10) I felt fearful & $\circ$ & $\circ$ & $\circ$ & $\circ$ \\
\hline 11) My sleep was restless & $\circ$ & O & $\circ$ & $\circ$ \\
\hline 12) I was happy & O & ○ & O & $\circ$ \\
\hline 13) I talked less than usual & $\circ$ & $\circ$ & $\circ$ & $\circ$ \\
\hline 14) I felt lonely & $\circ$ & o & $\circ$ & o \\
\hline 15) People were unfriendly & ○ & ○ & O & $\circ$ \\
\hline 16) I enjoyed life & ○ & $\circ$ & $\circ$ & ○ \\
\hline 17) I had crying spells & $\circ$ & $\circ$ & $\circ$ & $\circ$ \\
\hline 18) I felt sad & $\circ$ & $\circ$ & $\circ$ & $\circ$ \\
\hline 19) I felt that people disliked me & $\circ$ & O & $\circ$ & 0 \\
\hline 20) I could not get "going" & o & $\mathrm{O}$ & $\circ$ & 0 \\
\hline
\end{tabular}




\section{APPENDIX H}

CES-Depression Scale (CES-D), Radloff, 1977: Spanish

INSTRUCCIONES: Por favor indique con que frecuencia usted se ha sentido así en la última semana.

\begin{tabular}{|c|c|c|c|c|}
\hline $\begin{array}{l}\text { Durante la última semana: } \\
\text { (Una respuesta por cada pregunta) }\end{array}$ & $\begin{array}{c}\text { Casi } \\
\text { Nunca/ } \\
\text { Nunca } \\
\text { (menos } \\
\text { de } 1 \\
\text { día) } \\
\end{array}$ & $\begin{array}{c}\text { Muy } \\
\text { Poco } \\
\text { (1-2 } \\
\text { días) }\end{array}$ & $\begin{array}{l}\text { Algunas } \\
\text { veces/poco } \\
\text { (3-4 días) }\end{array}$ & $\begin{array}{c}\text { Casi } \\
\text { Siempre/ } \\
\text { Siempre } \\
\text { (5-7 } \\
\text { días) }\end{array}$ \\
\hline $\begin{array}{l}\text { 1) Me han molestado cosas que } \\
\text { normalmente no me molestan. }\end{array}$ & o & o & o & ○ \\
\hline $\begin{array}{l}\text { 2) No tuve ganas de comer, no tuve } \\
\text { apetito. }\end{array}$ & o & ○ & ○ & o \\
\hline $\begin{array}{l}\text { 3) No he podido superar el sentirme } \\
\text { triste ni siquiera con la ayuda de mi } \\
\text { familia o mis amigos. }\end{array}$ & $\circ$ & ○ & $\circ$ & o \\
\hline 4) Me sentí de igual valor que otra gente. & $\mathrm{o}$ & 0 & 0 & $\mathrm{o}$ \\
\hline $\begin{array}{l}\text { 5) Tuve problemas en concentrarme con } \\
\text { lo que hacía. }\end{array}$ & 0 & 0 & 0 & 0 \\
\hline 6) Me sentí deprimida/o. & o & o & o & o \\
\hline $\begin{array}{l}\text { 7) Sentí que todo lo que hacía era un } \\
\text { esfuerzo. }\end{array}$ & $\circ$ & ○ & o & ○ \\
\hline 8) Me sentí esperanzada/o en el futuro. & 0 & 0 & 0 & $\mathrm{O}$ \\
\hline 9) Sentí que mi vida ha sido un fracaso. & O & O & O & o \\
\hline 10) Me sentí con miedo. & $\mathrm{o}$ & $\mathrm{o}$ & $\mathrm{o}$ & $\mathrm{o}$ \\
\hline 11) No dormí bien. & $\mathrm{O}$ & $\mathrm{O}$ & $\mathrm{O}$ & $\circ$ \\
\hline 12) Me sentí feliz. & $\mathrm{o}$ & $\mathrm{o}$ & o & O \\
\hline 13) Hablé menos que de costumbre. & $\mathrm{o}$ & $\mathrm{o}$ & $\mathrm{o}$ & 0 \\
\hline 14) Me sentí sola/o. & O & O & O & O \\
\hline 15) La gente fue poco amable. & $\mathrm{o}$ & $\mathrm{o}$ & $\mathrm{o}$ & $\mathrm{o}$ \\
\hline 16) Disfruté de la vida. & $\mathrm{O}$ & $\mathrm{O}$ & 0 & $\circ$ \\
\hline 17) Lloré a veces. & 0 & 0 & 0 & O \\
\hline 18) Me sentí triste. & O & O & O & 0 \\
\hline 19) Sentí que la gente no me quiere. & 0 & 0 & 0 & 0 \\
\hline 20) No tuve ánimo. & o & O & O & o \\
\hline
\end{tabular}




\section{APPENDIX I}

Behavioral Risk Assessment Tool (CDC, 2009): English

Now I am going to ask you a few questions about your risk for HIV.

\section{During the last three months, have you...}

1) Been homeless?
o Yes
o No
o Refused

2) Been in alcohol or drug treatment?
o Yes
o No
o Refused

3) Had sex while high on drugs or alcohol?
o Yes
o No
o Not sure
o Refused

4) Had sex to get Money, drugs, shelter, etc.?
o Yes
o No
o Refused

5) Paid for sex with money or drugs?
o Yes
o No
o Refused

6) Been paid for sex with money or drugs?
o Yes
o No
o Refused

7) Had sex with someone who injects drugs?
o Yes
o No
o Not sure
o Refused

8) Had sex with a man who has sex with other men?
o Yes
o No
o Refused 
9) Had sex with a woman?
o Yes
o No
o Refused

10) Been diagnosed with Hepatitis $C$ ?
o Yes
o No
o Not sure
o Refused

11) Been diagnosed with a sexually transmitted disease (Syphilis, Chlamydia, gonorrhea, etc.)?
o Yes
o No
o Not sure
o Refused

12) Been in the correction system
o Yes
o No
o Refused

\section{During your life, have you...}

13) Ever injected drugs including hormones?
o Yes
o No
o Not sure
o Refused

14) Ever been in alcohol or drug treatment?
o Yes
o No
o Refused

15) Ever had sex against your will?
o Yes
o No
o Refused

16) In the past 3 months, have you had vaginal, oral, or anal sex?
o Yes
o No
o Refused

17) In the past 3 months, have you had sex with a man?
o Yes
o No
o Refused 
18) How many men?

19) How many women?

20) How many transgender persons?

In the past 3 months, what type of sex have you had?

21) Vaginal sex?
o Yes
o No
o Refused

22) How often did you use a condom during vaginal sex?
o Not applicable
o Always
o Usually
o Sometimes
o Occasionally
o Never

23) Performed anal sex as a top?
o Yes
O No
o Refused

24) When you performed anal sex as a top, how often did you use a condom?

o Not applicable

o Always

o Usually

o Sometimes

o Occasionally

o Never

25) Received anal sex as a bottom?
o Yes
o No
o Refused

26) When you performed or gave oral sex, how often did you use a condom?
o Not applicable
o Always
o Usually
o Sometimes
o Occasionally
o Never 
27) Performed or gave oral sex?
o Yes
o No
o Refused

28) When you performed or gave oral sex, how often did you use a condom?

o Not applicable

o Always

o Usually

o Sometimes

o Occasionally

o Never

29) Received oral sex?
o Yes
o No
o Refused

30) When you received oral sex, how often did you use a condom?
o Not applicable
o Always
o Usually
o Sometimes
o Occasionally
o Never

31) In the past three months have you had unprotected sex (sex without a condom) with someone who has HIV?
o Yes
0 No
o Refused

32) In the past 3 months have you had unprotected sex (sex without a condom) with someone whose HIV status you didn't know?
o Yes
o No
o Refused

33) Do you have a spouse or main partner?
o Yes
o No
o Refused

34) If yes, how long have you been with this partner?

35) Does your partner have HIV?
o Yes
o No
o Refused 
36) In the past 30 days, have you used any non-injected drugs?

$$
\begin{array}{ll}
\text { o } & \text { Yes } \\
\text { o } & \text { No } \\
\text { o } & \text { Refused }
\end{array}
$$

37) In the past 30 days, have you injected any drugs or medications including hormones?
o Yes
o No
o Refused

\begin{tabular}{|l|c|c|c|c|c|}
\hline $\begin{array}{l}\text { Have you used the following drugs } \\
\text { in the past 30 days? }\end{array}$ & Yes & $\begin{array}{c}\text { If so, how many } \\
\text { times in the past } \\
\text { 30 days? }\end{array}$ & No & $\begin{array}{c}\text { Not } \\
\text { sure }\end{array}$ & Refused \\
\hline 38) Crack & $\circ$ & & $\circ$ & $\circ$ & $\circ$ \\
\hline 39) Cocaine & $\circ$ & & $\circ$ & $\circ$ & $\circ$ \\
\hline 40) Heroine & $\circ$ & & $\circ$ & $\circ$ & $\circ$ \\
\hline 41) Amphetamines (Speed, Crystal) & $\circ$ & & $\circ$ & $\circ$ & $\circ$ \\
\hline 42) Amil nitrates (poppers) & $\circ$ & & $\circ$ & $\circ$ & $\circ$ \\
\hline $\begin{array}{l}\text { 43) Club drugs (Ecstasy, Special K, } \\
\text { GHB) }\end{array}$ & $\circ$ & & $\circ$ & $\circ$ & $\circ$ \\
\hline 44) Marijuana & $\circ$ & & $\circ$ & $\circ$ & $\circ$ \\
\hline $\begin{array}{l}\text { 45) Prescription medications } \\
\text { (oxycotin, oxycodone) }\end{array}$ & $\circ$ & & $\circ$ & $\circ$ & $\circ$ \\
\hline 46) Alcohol & $\circ$ & & $\circ$ & $\circ$ & $\circ$ \\
\hline $\begin{array}{l}\text { 47) Tobacco (cigarrettes, snuff, } \\
\text { etc.) }\end{array}$ & $\circ$ & & $\circ$ & $\circ$ & $\circ$ \\
\hline
\end{tabular}

\begin{tabular}{|l|c|c|c|c|c|}
\hline $\begin{array}{l}\text { Have you used any injection drugs } \\
\text { in the last 30 days? }\end{array}$ & Yes & $\begin{array}{c}\text { If you have used, } \\
\text { how many times } \\
\text { in the last 30 } \\
\text { days? }\end{array}$ & No & $\begin{array}{c}\text { Not } \\
\text { sure }\end{array}$ & Refused \\
\hline 48) Crack & $\circ$ & & $\circ$ & $\circ$ & $\circ$ \\
\hline 49) Cocaine & $\circ$ & & $\circ$ & $\circ$ & $\circ$ \\
\hline 50) Heroin & $\circ$ & & $\circ$ & $\circ$ & $\circ$ \\
\hline 51) Amphetamines (Speed, Crystal) & $\circ$ & & $\circ$ & $\circ$ & $\circ$ \\
\hline 52) Steroids & $\circ$ & & $\circ$ & $\circ$ & $\circ$ \\
\hline 53) Insuline & $\circ$ & & $\circ$ & $\circ$ & $\circ$ \\
\hline 54) Hormones & $\circ$ & & $\circ$ & $\circ$ & $\circ$ \\
\hline $\begin{array}{c}\text { 55) Prescription medications } \\
\text { (oxycotin, oxycodone) }\end{array}$ & $\circ$ & & $\circ$ & $\circ$ & $\circ$ \\
\hline
\end{tabular}




\begin{tabular}{|l|c|c|c|c|}
\hline $\begin{array}{l}\text { If you have injected drugs in the past 30 days, } \\
\text { what type of needle did you use? }\end{array}$ & Yes & No & Refused & $\begin{array}{c}\text { Not } \\
\text { applicable }\end{array}$ \\
\hline 56) New & $\circ$ & $\circ$ & $\circ$ & $\circ$ \\
\hline 57) Used (disinfected with bleach) & $\circ$ & $\circ$ & $\circ$ & $\circ$ \\
\hline 58) Shared with someone who used it before you & $\circ$ & $\circ$ & $\circ$ & $\circ$ \\
\hline 59) Shared with someone who used it after you & $\circ$ & $\circ$ & $\circ$ & $\circ$ \\
\hline 60) Reused my own needle & $\circ$ & $\circ$ & $\circ$ & $\circ$ \\
\hline 61$)$ I don't know where the needle came from & $\circ$ & $\circ$ & $\circ$ & $\circ$ \\
\hline
\end{tabular}




\section{APPENDIX J}

Behavioral Risk Assessment Tool (CDC, 2009): Spanish

Ahora le voy a hacer algunas preguntas a su riesgo de contraer VIH.

En los últimos 3 meses, ha usted...........

1) ¿Vivido en la calle?
O Si
o No
o Rehuso

2) ¿Estado bajo tratamiento por alcoholismo o abuso de drogas?
O $\mathrm{Si}$
o No
o Rehuso

3) ¿Tenido relaciones sexuales bajo la influencia de alcohol o drogas?
o $\mathrm{Si}$
o No
o No estoy seguro
o Rehuso

4) ¿Tenido relaciones sexuales por dinero, drogas, albergue etc.?
O Si
o No
o Rehuso

5) ¿Pagado por sexo con dinero o drogas?
O $\mathrm{Si}$
O No
o Rehuso

6) ¿Recibido dinero o drogas a cambio de actos sexuales?
O Si
O No
o Rehuso

7) Tenido relaciones sexuales con alguien que se inyecta drogas intravenosas?
O Si
o No
o No estoy seguro
o Rehuso 
8) ¿Tenido relaciones sexuales con un hombre que tiene relaciones sexuales con hombres?
O $\mathrm{Si}$
o No
o Rehuso

9) ¿Tenido relaciones sexuales con mujeres?
O $\mathrm{Si}$
o No
o Rehuso

10) ¿Sido diagnosticado con hepatitis C?
O Si
o No
o No estoy seguro
o Rehuso

11) ¿Sido diagnosticado con enfermedades de transmisión sexual? (Sifilis, Clamidia, gonorrea, etc.)
O $\mathrm{Si}$
o No
o No estoy seguro
o Rehuso

12) ¿Pasado por el sistema correccional? (libertad condicional, etc.)
o $\mathrm{Si}$
o No
o Rehuso

\section{Durante su vida, ha usted...}

13) ¿Alguna vez inyectado drogas incluyendo hormonas?
O Si
o No
o No estoy seguro
o Rehuso

14) ¿Alguna vez estado bajo tratamiento por alcoholismo o por uso de drogas?
O Si
o No
o Rehuso

15) ¿Alguna vez tenido relaciones sexuales en contra de su voluntad?
O Si
O No
o Rehuso 
16) ¿En los últimos 3 meses, ha tenido sexo vaginal, oral o anal?

$$
\begin{array}{ll}
\text { o } & \text { Si } \\
\text { o } & \text { No } \\
\text { o } & \text { Rehuso }
\end{array}
$$

17) ¿En los últimos 3 meses, ha tenido relaciones sexuales con un hombre?

$$
\begin{array}{ll}
\text { o } & \text { Si } \\
\text { o } & \text { No } \\
\text { o } & \text { Rehuso }
\end{array}
$$

18) ¿Cuantos hombres?

19) ¿Cuantas mujeres?

20) ¿Cuantas personas transgenero?

\section{En los últimos 3 meses, que tipo de relaciones sexuales ha tenido?}

21) Sexo vaginal?
O Si
o No
o Rehuso

22) ¿Cuán frecuente uso un condón durante el sexo vaginal?
o No applicable
o Siempre
o Usalmente
o Alguna veces
o Ocasionalmente
o Nunca

23) ¿Realizado sexo anal como el activo?
o $\mathrm{Si}$
o No
o Rehuso

24) ¿Cuando realizó sexo anal como el activo, cuan frecuente uso un condón?
o No applicable
o Siempre
o Usalmente
o Alguna veces
o Ocasionalmente
o Nunca

25) ¿Recibido sexo anal como el pasivo?
o $\mathrm{Si}$
o No
o Rehuso 
26) ¿Cuando recibió sexo anal como el pasivo, ¿cuan frecuente uso un condón?
o No applicable
o Siempre
o Usalmente
o Alguna veces
o Ocasionalmente
o Nunca

27) ¿Realizo o dio sexo oral?
o $\mathrm{Si}$
o No
o Rehuso

28) ¿Cuando realizo o dio sexo oral, cuan frecuente uso un condón?
o No applicable
o Siempre
o Usalmente
o Alguna veces
o Ocasionalmente
o Nunca

29) ¿Recibido sexo oral?
o $\mathrm{Si}$
o No
o Rehuso

30) Cuando recibió sexo oral, ¿¿cuan frecuente uso un condón?
o No applicable
o Siempre
o Usalmente
o Alguna veces
o Ocasionalmente
o Nunca

31) ¿En los últimos tres meses ha tenido sexo sin protección (sexo sin usar condón) con alguien que esta infectado con el VIH?
O Si
0 No
o Rehuso

32) ¿En los últimos tres meses ha tenido sexo sin protección (sexo sin usar condón) con alguien del cual desconoce si esta infectado con el VIH o no?
o $\mathrm{Si}$
0 No
o Rehuso

33) ¿Tiene esposo(a) o pareja?
o $\mathrm{Si}$
o No
o Rehuso 
34) ¿Si tiene, por cuanto tiempo ha estado con esta persona?

35) ¿Su pareja esta infectado(a) con el VIH?
o Si
o No
o Rehuso

36) ¿En los últimos 30 días, ha usado alguna droga no inyectada?
O Si
o No
o Rehuso

37) ¿En los últimos 30 días, se ha inyectado alguna droga o medicamento incluyendo hormonas?
O Si
o No
o Rehuso

\begin{tabular}{|l|c|c|c|c|c|}
\hline $\begin{array}{l}\text { ¿Ha usado alguna de las } \\
\text { siguientes drogas en los últimos } \\
\text { 30 días? }\end{array}$ & Si & $\begin{array}{c}\text { Si ha usado, } \\
\text { cuantas veces } \\
\text { en los últimos } \\
\text { 30 días? }\end{array}$ & No & $\begin{array}{c}\text { No estoy } \\
\text { seguro(a) }\end{array}$ & Rehúso \\
\hline 38) Crack & $\circ$ & & $\circ$ & $\circ$ & $\circ$ \\
\hline 39) Cocaína & $\circ$ & & $\circ$ & $\circ$ & $\circ$ \\
\hline 40) Heroína & $\circ$ & & $\circ$ & $\circ$ & $\circ$ \\
\hline 41) Anfetaminas & $\circ$ & & $\circ$ & $\circ$ & $\circ$ \\
\hline 42) Nitrato de amilo (poppers) & $\circ$ & & $\circ$ & $\circ$ & $\circ$ \\
\hline $\begin{array}{l}\text { 43) Drogas recreativas (Ecstasy, } \\
\text { Special K, GHB) }\end{array}$ & $\circ$ & & $\circ$ & $\circ$ & $\circ$ \\
\hline 44) Marihuana & $\circ$ & & $\circ$ & $\circ$ & $\circ$ \\
\hline $\begin{array}{l}\text { 45) Medicamentos recetados } \\
\text { (codeína, morfina) }\end{array}$ & $\circ$ & & $\circ$ & $\circ$ & $\circ$ \\
\hline 46) Alcohol & $\circ$ & & $\circ$ & $\circ$ & $\circ$ \\
\hline $\begin{array}{l}\text { 47) Tabaco (cigarrillos, puros, } \\
\text { etc.) }\end{array}$ & $\circ$ & & $\circ$ & $\circ$ & $\circ$ \\
\hline
\end{tabular}




\begin{tabular}{|l|c|c|c|c|c|}
\hline $\begin{array}{l}\text { ¿Ha usado alguna de las } \\
\text { siguientes drogas inyectadas en } \\
\text { los últimos 30 días? }\end{array}$ & $\mathrm{Si}$ & $\begin{array}{l}\text { Si ha usado, } \\
\text { cuantas veces } \\
\text { en los últimos } \\
\text { 30 días? }\end{array}$ & No & $\begin{array}{c}\text { No estoy } \\
\text { seguro(a) }\end{array}$ & Rehúso \\
\hline 48) Crack & $\circ$ & & $\circ$ & $\circ$ & $\circ$ \\
\hline 49) Cocaína & $\circ$ & & $\circ$ & $\circ$ & $\circ$ \\
\hline 50) Heroína & $\circ$ & & $\circ$ & $\circ$ & $\circ$ \\
\hline 51) Anfetaminas (speed, crystal) & $\circ$ & & $\circ$ & $\circ$ & $\circ$ \\
\hline 52) Esteroides & $\circ$ & & $\circ$ & $\circ$ & $\circ$ \\
\hline 53) Insulina & $\circ$ & & $\circ$ & $\circ$ & $\circ$ \\
\hline 54. Hormonas & $\circ$ & & $\circ$ & $\circ$ & $\circ$ \\
\hline $\begin{array}{l}\text { 55) Medicamentos recetados } \\
\text { (codeína, morfina) }\end{array}$ & $\circ$ & & $\circ$ & $\circ$ & $\circ$ \\
\hline
\end{tabular}

\begin{tabular}{|l|c|c|c|c|}
\hline $\begin{array}{l}\text { ¿Se ha inyectado alguna droga en los últimos 30 } \\
\text { días, que tipo de agujas utilizo? }\end{array}$ & Si & No & Rehúso & N/A \\
\hline 56) Nueva & $\circ$ & $\circ$ & $\circ$ & $\circ$ \\
\hline 57) Usada (desinfectada con cloro) & $\circ$ & $\circ$ & $\circ$ & $\circ$ \\
\hline 58) Compartida (alguien la uso antes que yo) & $\circ$ & $\circ$ & $\circ$ & $\circ$ \\
\hline 59) Compartida (alguien la uso después de mi) & $\circ$ & $\circ$ & $\circ$ & $\circ$ \\
\hline 60) Reusé mi propia aguja & $\circ$ & $\circ$ & $\circ$ & $\circ$ \\
\hline 61) No se de donde vino la aguja & $\circ$ & $\circ$ & $\circ$ & $\circ$ \\
\hline
\end{tabular}




\section{CURRICULUM VITAE}

\section{BEATRIZ VALDES}

Education/Experience/Professional:

Florida International University, Miami, Florida 33181

Doctor of Philosophy in Nursing Program; PhD, Spring 2016

Certified Healthcare Simulator Educator (CHSE)

Certificate \# 161092, Expiration 4/19/2019

University of Phoenix, Plantation, FL 33324

Master of Science in Nursing - MSN and Master in Business for Healthcare

Administration- MBA, 2004

University of Miami, Coral Gables, FL 33146

Baccalaureate in the Science of Nursing- BSN, 1999

Miami-Dade Community College, Miami, FL 33136

Associates Degree in Science - Nursing, 1993

Lindsey Hopkins Technical Education Center, Miami, FL 33136

Practical Nurse Certification, 1990

2012 to Present Nurse Specialist, School of Nursing and Health Studies, International Academy for Clinical Simulation and Research, University of Miami, Coral Gables, FL 33146

2016 Recipient of the 2016 Sigma Theta Tau International "Rising Stars of Research and Scholarship" poster presentation in Cape Town South Africa, July 2016

2015 Recipient Sigma Theta Tau International, Beta Tau Chapter PhD Dissertation Research Award \$1,500.00

2015 Peer-Reviewer for Abstracts submitted to the 16th International Meeting on Simulation in Healthcare (IMSH 2016)

2015 Peer-Reviewer for Abstracts submitted to the Southern Nursing Research Society's 29th Annual Conference 2015 (SNRS 2015)

2014-2015 Robert Wood Johnson Foundation New Careers in Nursing Scholars Program Mentor 
2010 - 2012 Adjunct Clinical Professor, College of Nursing and Health Sciences, Florida International University, Miami FL 33199

2009 - 2012 Adjunct Clinical Professor, College of Nursing and Health Sciences, Barry University, Miami Shores FL 33161

2008

Nurse Educator of the Year, Jackson Health System.

2006- 2007 Registered Nurses’ Association of Ontario (RNAO) Collaborative Practice Among Nursing Teams, Guidelines Stakeholder Nurse Practice Guideline

1999-Present Sigma Theta Tau International Honor Society Scholar

\section{PUBLICATIONS}

Everett-Thomas, R., Turnbull-Horton, V., Valdes, B., Valdes, G., Rosen, L. F., \& Birnbach, D. J. (2016). The influence of high fidelity simulation on first responders retention of CPR knowledge. Applied Nursing Research, 30, 94-97. doi:10.1016/j.apnr.2015.11.005

Jones, S. G., Fenkl, E. A., Patsdaughter, C. A., Chadwell, K., \& Valdes, B. (2015). The perceived effects of condoms on sexual experience: A comparison of Hispanic and non-Hispanic men. Hispanic Health Care International. doi:10.1891/15404153.13.3.61

De Santis, J. P., Valdes, B., Provencio-Vasquez, E., \& Gattamorta, K. A. (2015). A comparison by sexual orientation of sexual health and sexual behaviors among Hispanic men. Sexuality Research and Social Policy, 12(1), 15-23. doi:10.1007/s13178-014-0170-9

De Santis, J. P., Valdes, B., Provencio-Vasquez, E., Patsdaughter, C. A., \& Gattamorta, K.A. (2014). A comparison of substance use behaviors of Hispanic men by sexual orientation. Nursing and Health, 2(1), 9-17. doi:10.13189/nh.2014.020102

Everett-Thomas, R., Valdes, B., Valdes, G., Shekhter, I., Fitzpatrick, M., Rosen, L. F., Arheart, K. L., \& Birnbach, D. J. (2014). Using simulation technology to identify gaps between education and practice among new graduate nurses. The Journal of Continuing Education in Nursing, 45 (X), 1-7. doi:10.3928/00220124-2014112201

Valdes, B. (2014). [Review of the book The velvet rage: Overcoming the pain of growing up gay in a straight man's world (2nd ed.) by A. Downs]. Journal of Homosexuality, 61(2), 354-360. doi:10.1080/00918369.2013.839922 